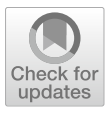

Cite as

Nano-Micro Lett.

(2021) 13:156

Received: 26 April 2021

Accepted: 6 June 2021

Published online: 15 July 2021

(C) The Author(s) 2021

\section{Differences and Similarities of Photocatalysis and Electrocatalysis in Two-Dimensional Nanomaterials: Strategies, Traps, Applications and Challenges}

\author{
Weiqi Qian ${ }^{1,5}$, Suwen Xu ${ }^{1,2}$, Xiaoming Zhang ${ }^{2},{\text { Chuanbo } \mathrm{Li}^{2}{ }^{凶}, \text { Weiyou Yang }}^{3}$, \\ Chris R. Bowen ${ }^{4}$, Ya Yang ${ }^{1,5,6} \bowtie$
}

\title{
HIGHLIGHTS
}

- This review focuses on the differences and similarities of photocatalysis and electrocatalysis in the latest 2D nanomaterials.

- Strategies and traps for performance enhancement of 2D nanocatalysts are highlighted.

- Challenges, future directions and applications for new photocatalysis and electrocatalysis exploiting 2D nanomaterials are suggested.

\begin{abstract}
Photocatalysis and electrocatalysis have been essential parts of electrochemical processes for over half a century. Recent progress in the controllable synthesis of 2D nanomaterials has exhibited enhanced catalytic performance compared to bulk materials. This has led to significant interest in the exploitation of 2D nanomaterials for catalysis. There have been a variety of excellent reviews on 2D nanomaterials for catalysis, but related issues of differences and similarities between photocatalysis and electrocatalysis in 2D nanomaterials are still vacant. Here, we provide a comprehensive overview on the differences and similarities of photocatalysis and electrocatalysis in the latest 2D nanomaterials. Strategies and traps for performance enhancement of 2D nanocatalysts are highlighted, which point out the differences and similarities of series issues for photocatalysis and electrocatalysis. In addition, 2D nanocatalysts and their catalytic applications are discussed. Finally, opportunities, challenges and development directions for 2D nanocatalysts are described. The intention of this review is to inspire

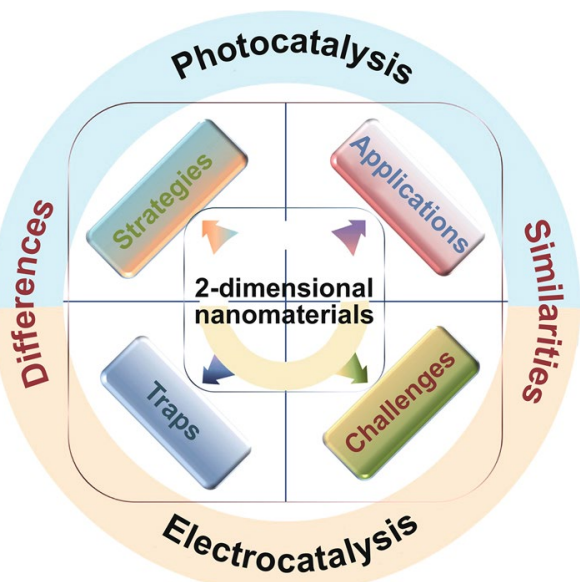
and direct interest in this research realm for the creation of future 2D nanomaterials for photocatalysis and electrocatalysis.

KEYWORDS 2D nanomaterials; Photocatalysis; Electrocatalysis; Electrochemistry; Photoelectrochemistry

Weiqi Qian and Suwen Xu contributed equally to this work.

$\triangle$ Chuanbo Li, cbli@muc.edu.cn; Weiyou Yang, weiyouyang@tsinghua.org.cn; Ya Yang, yayang@binn.cas.cn

1 Beijing Key Laboratory of Micro-Nano Energy and Sensor, CAS Center for Excellence in Nanoscience, Beijing Institute of Nanoenergy and Nanosystems, Chinese Academy of Sciences, Beijing 101400, People's Republic of China

2 Optoelectronics Research Center, School of Science, College of Life and Environmental Sciences, Minzu University of China, Beijing 100081 , People's Republic of China

3 Institute of Materials, Ningbo University of Technology, Ningbo 315016, People's Republic of China

4 Department of Mechanical Engineering, University of Bath, Bath BA2 7AK, UK

5 School of Nanoscience and Technology, University of Chinese Academy of Sciences, Beijing 100049, People's Republic of China

${ }^{6}$ Center on Nanoenergy Research, School of Physical Science and Technology, Guangxi University, Nanning 530004, People’s Republic of China
\end{abstract}




\section{Introduction}

Developing new forms of renewable energy generation can be considered the most promising strategy to tackle the world's growing environmental challenges and the global energy crisis [1-5]. Catalysis has received intensive interest in sustainable energy development and environmental remediation since the work of Fujishima et al. in 1972, due to their pioneering work on titanium dioxide $\left(\mathrm{TiO}_{2}\right)$ photoelectrodes [6]. Generally, photocatalysis, as one of common catalysis, is the utilization of semiconductor photocatalysts to accelerate photochemical reactions, where the photogenerated separated electron-hole pairs participate in the following oxidation-reduction reactions [7-9]. Electrocatalysis is a specific form of catalysis that accelerates charge transfer between the electrodes and the electrolyte interfaces [10,11], where most commonly electrocatalysts are a kind of catalysts attached on the surface of electrodes or as the electrode surface that are largely beneficial for electron transfer between reactants and electrodes [12-14]. So far, photocatalysis and electrocatalysis are often essential parts of chemical processes for water splitting and pollution treatment, which are important reactions for harvesting ubiquitous forms of ambient energy [15-23].

As the gradually deepening process of two-dimensional (2D) nanomaterials in molecular design and synthesis [24-29], a number of 2D nanomaterials have been used as a catalyst in their three-dimensional (3D) bulk form; however, their performance as a photocatalysis or electrocatalysis continues to suffer from a low efficiency in terms of charge separation and low transfer kinetics compared to 2D nanomaterials [12, 30-32]. As an example, the traditional design of graphitic carbon nitride $\left(\mathrm{g}-\mathrm{C}_{3} \mathrm{~N}_{4}\right)$-based materials has considered bulk nanosheets [33]. However, due to the strong stacking forces between atom layers, the use of a bulk nanomaterial leads to a low surface reactivity, a high probability of charge recombination and poor solar absorptivity $[34,35]$. It can therefore be assumed that the dimensionality and surface characteristics play an important role in determining the key catalytic properties for practical applications and the optimum fabrication process of the material [12]. Hence, research that aims to develop atomically thin 2D catalysts with enhanced charge carrier dynamics and improved mobility continues to attract interest.

To enhance the photocatalytic and electrocatalytic performance, growing attention has been attached to the development of 2D nanomaterials with good electrical conductivity and large surface area [34]. In contrast to conventional $3 \mathrm{D}$ bulk nanomaterials, these atomically thin $2 \mathrm{D}$ nanomaterials have attracted attention in environmental and energy-related research sectors as a result of their extraordinary stability and activity, often on account of their high specific surface area, robust mechanical structure and excellent electrical conductivity. In addition, 2D nanomaterials have been pursued as economical alternatives to more expensive precious metals, such as platinum and rhodium [35]. Recent progress in multiple atomically thin 2D nanomaterials has broken new ground; there have been rapid developments in the synthesis of 2D nanomaterials, and their resulting properties, surface chemistry and catalytic applications [36]. To date, a detailed understanding on the rational design and construction of efficient 2D nanomaterial-based catalysts as well as the issues associated with industrial scale applications is still not comprehensive enough [37]. Therefore, comprehensive overview is still needed to provide new insights on the fabrication and application of recent developments, and fundamental studies are needed for clear reaction processes to improve catalytic performance for applications that are ripe for industrial exploitation [38-40]. There have been a variety of excellent reviews on 2D nanomaterials for catalysis [10, 34, 41-44]. However, related issues of differences and similarities between photocatalysis and electrocatalysis in 2D nanomaterials are still vacant, but worthy of great attention since demands to generate exceptional catalytic activities are strongly different for photocatalytic and electrocatalytic reaction systems.

Herein, a comprehensive overview will focus on the differences and similarities of photocatalysis and electrocatalysis in the latest 2D nanomaterials. A comparison of differences and similarities of photocatalysis and electrocatalysis in 2D nanomaterials is concluded in Table 1 . We will begin with strategies for performance enhancement of 2D nanocatalysts as a highlight, which will point out the differences and similarities of photocatalysis and electrocatalysis. Then, the traps of catalytic-related systems in 2D nanomaterials will be emphasized to direct related experiment processing to consider and exclude several details for all-round research. Moreover, an introduction of typical 2D nanocatalysts that have long been considered research hotspots will be exhibited, including their classification, structures, synthesis approaches and characterizations. The 
Table 1 Comparison of differences and similarities for photocatalysis and electrocatalysis in 2D nanomaterials

\begin{tabular}{|c|c|c|c|}
\hline & & Photocatalysis & Electrocatalysis \\
\hline \multirow[t]{3}{*}{ Characteristics of catalysts } & Type & $\begin{array}{l}\text { 2D nanomaterial } \\
\text { powders or compos- } \\
\text { ite thin films }\end{array}$ & $\begin{array}{l}\text { 2D nanomaterial thin films or powders loaded on an electrode } \\
\text { surface }\end{array}$ \\
\hline & Hydrophilicity & Hydrophilic & Hydrophobic or hydrophilic \\
\hline & Recyclability & $\begin{array}{l}\text { Complex for pow- } \\
\text { ders, feasible for } \\
\text { thin films }\end{array}$ & Feasible \\
\hline \multirow[t]{3}{*}{ Conditions of reaction systems } & Energy input & Solar energy & Externally applied electric bias \\
\hline & Configuration & One reaction chamber & $\begin{array}{l}\text { One reaction chamber or two chambers separated by mem- } \\
\text { brane }\end{array}$ \\
\hline & Charge transfer pathway & Relatively short & Relatively long \\
\hline \multirow[t]{2}{*}{ Benefits } & Cost & Low & High including expensive electrode and electric energy \\
\hline & Efficiency & Low & Relatively high \\
\hline
\end{tabular}

catalytic applications of 2D nanomaterials in the areas of environmental treatment and biochemical technologies will be discussed. Finally, opportunities, challenges and development directions for 2D catalysts for photocatalysis and electrocatalysis will be described. The intention of this review is to inspire and direct interest in this research realm for the creation of future 2D nanomaterials for photocatalysis and electrocatalysis.

\section{Strategies for Catalytic Performance Enhancement of 2D Nanomaterials}

Carrier separation and transfer kinetics are generally considered as the most significant issues for improving performance for photocatalysis and electrocatalysis [45], which can be considerably related to structure-activity correlation of catalysts [46, 47]. To date, 2D layered nanomaterials including graphene and graphite-like materials continue to suffer from a variety of issues that restrict their functionality and practicability in applications related to semiconductors, sensors and catalysis [37]. Therefore, diverse and abundant strategies must be explored and analyzed to produce layered nanomaterial-based catalysts with enhanced photocatalysis and electrocatalysis performances. Obviously, 2D nanocatalysts show a variety of advantage contrasted to 3D bulk catalysts, which will be presented in the following subsection. In addition, differences and similarities in strategies of photocatalysis and electrocatalysis will be, respectively, discussed and all of these contents are concluded in Fig. 1.

\subsection{Superiority of 2D Nanocatalysts}

Structure-activity correlation of 2D nanocatalysts illustrates a significant influence of geometric configurations in catalytic performance [46-48], which can be attributed to the unique characteristics $2 \mathrm{D}$ nanocatalysts possessing as follows:

(1) Surface-active sites. The general geometric configurations of 2D nanocatalysts result in large specific surface area, which can lead to large exposed lattice planes and surface-active sites with high density for further. High density surface-active sites can enhance the catalytic reactions on the material surface. Another approach to increase the exposure of surface-active sites is to decrease 2D nanocatalysts' lateral size [49]. For example, it is reported that ultrasmall molybdenum disulfide $\left(\mathrm{MoS}_{2}\right)$ exhibits enhanced hydrogen evolution reaction (HER) performance than bulk $\mathrm{MoS}_{2}$, which can be attributed to an enrichment of active sulfur edges for HER [50].

(2) Carrier mobility. High electron mobility has been extensively observed in various ultrathin 2D nanomaterials, including graphene, transition metal dichalcogenides (TMDs) and black phosphorous (BP) nanosheets $[30,51]$. For instance, the reported mobility of graphene and $\mathrm{MoS}_{2}$ ranges in $10^{2} \sim 10^{4} \mathrm{~cm}^{2} \mathrm{~V}^{-1} \mathrm{~s}^{-1}[52-$ 56] and around $10^{1} \mathrm{~cm}^{2} \mathrm{~V}^{-1} \mathrm{~s}^{-1}$ [57-61], respectively. The unique ultrathin structure of $2 \mathrm{D}$ nanocatalysts provides relatively high charge migration due to short transport path and small basic resistance. Yu et al. observed an obvious drop of HER performance when $\mathrm{MoS}_{2}$ attached by additional atomic layer, which can be 


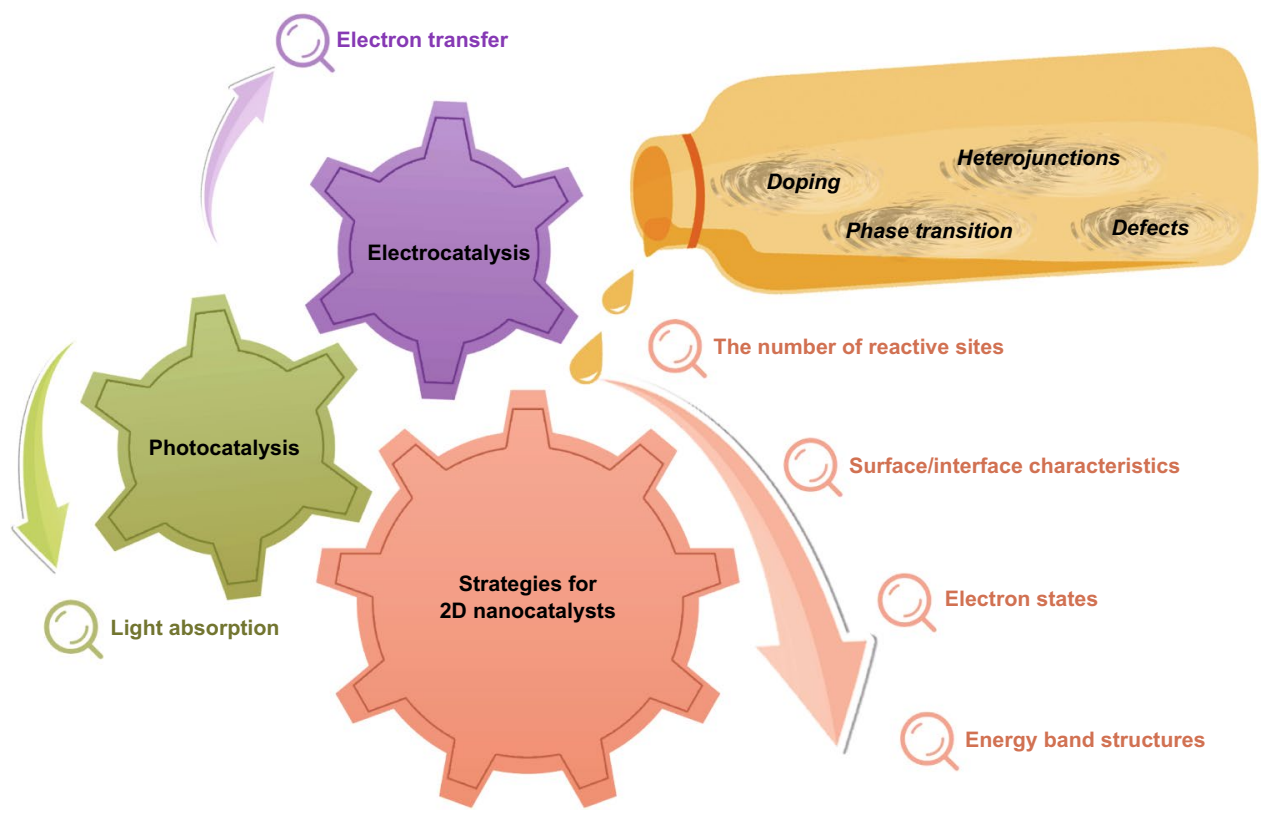

Fig. 1 A series of general strategies for 2D nanocatalysts cover the number of reactive sites, surface/interface characteristics, electron states and energy band structures. Specific strategies for photocatalysis and electrocatalysis are, respectively, on the basis of light absorption and electron transfer. Besides, approaches, such as doping, heterojunctions, phase transition and defects, perform the function as lubricants to realize the above-mentioned strategies for catalytic performance enhancement

interpreted as the restriction of electron mobility along a vertical direction between the material layers [62].

(3) Energy band structures. For a variety of 2D layered nanomaterials, changing number of layers in the crystal can tune the band gap, where the tunable band structure determines the light absorption properties of materials for photocatalysis. The band gap of $\mathrm{MoS}_{2}$ can be tuned via changing the number of cumulate layers, where the band gap of single-layered and few-layered $\mathrm{MoS}_{2}$ are $1.8 \sim 1.9$ and $1-1.2 \mathrm{eV}$, respectively [63]. The reported band gap of $\mathrm{g}-\mathrm{C}_{3} \mathrm{~N}_{4}$ can be tuned ranging from 1.6 to $-1.1 \mathrm{eV}$ versus normal hydrogen electrode (NHE) [64]. For bismuth-based 2D layered nanomaterials, the band gap can be manipulated ranging in $0.3-3.6 \mathrm{eV}$ via introducing various cations and anions into intrinsic structure, whose corresponding light response covers ultraviolet to near infrared $[65,66]$. In addition to the light absorption properties, the interfacial adsorption/ desorption free energy between catalysts and reactants can also be regulated via the tunable band structure as well as the changing electron distribution [67].

(4) Electronic properties. Through controlling the thickness of 2D nanocatalysts can realize the regulation of the electronic properties [68]. As reported, the electronic structures of 2D nanomaterials are able to regulate the bond strength between reactants and catalytic active sites and reduce the desorption kinetic barrier [69]. Fang's group reported a battery based on 2D mesoporous covalent organic frameworks (COFs) with superior areal capacitance, gravimetric power and maximum power density of $5.46 \mathrm{mF} \mathrm{cm}^{-2}, 55 \mathrm{Kw} \mathrm{kg}^{-1}$ and $4.1-5.4 \mathrm{~W} \mathrm{~cm}^{-3}$, respectively; this was two orders of magnitude better than conventional Li thin-film batteries [70]. Atomic ultrathin 2D nanocatalysts bring the benefits of abundant in-plane defects that are conducive to the electronic properties such as electrical conductivity [71], which improves the conduction of electricity energy sources generated during catalysis. Voiry et al. reported abundant defects of monolayered tungsten disulfide $\left(\mathrm{WS}_{2}\right)$ nanosheets are beneficial for HER, which related to the induced lattice distortions [72].

(5) Mechanical properties. 2D nanomaterials prone to possess prominent mechanical properties [73-75], which confer high catalyst durability that is a path to practical application for benefit of humankind. In addition, the robustness of 2D nanomaterials offers the possibility in the development of hybrid nanocatalysts for catalytic enhancement.

On the basis of the above concluded superiority of 2D nanocatalysts, great progress has been achieved in $2 \mathrm{D}$ 
nanomaterials for photocatalysis and electrocatalysis [10, $34,43,44]$. Nevertheless, the catalytic activity of 2D nanomaterials needs to be further enhanced, and there are a series of strategies gradually emerging [76-78], which will be suggested in the following subsections.

\subsection{Similarities in Strategies of Photocatalysis and Electrocatalysis}

For various strategies of photocatalysis and electrocatalysis in 2D nanomaterials, there are differences as well as similarities in specific catalytic systems. Herein, we will discuss the similarities in strategies of 2D nanocatalysts' photocatalysis and electrocatalysis.

In general, the similar strategies of 2D layered nanocatalysts for both photocatalytic and electrocatalytic enhancement can be concluded both kinetically and thermodynamically through regulating the following guidelines, such as (1) the number of reactive sites, (2) surface/interface characteristics, (3) electronic properties and (4) energy band structures, where these common guidelines can be promisingly realized via regulating morphology, doping, constructing heterostructures, importing defects and engineering phases, etc.

(1) The number of reactive sites. As above-mentioned in the last subsection, 2D nanocatalysts with a unique morphology of ultrathin atomic layers bring the benefits of the highest number of catalytic reactive sites according to the highest surface area in theory. Set 2D layered $\mathrm{MoS}_{2}$ nanosheets as an example, an enhanced electrocatalytic HER ability has been achieved by morphologically controlling the surface structure with the regulation of size and thickness to expose more electrocatalytic reactive sites [35]. For photocatalytic degradation reactions, Parzinger et al. demonstrated that monolayered $\mathrm{MoS}_{2}$ nanosheets' edges sites were more resistant than those of multilayered $\mathrm{MoS}_{2}$ nanosheets [79]. In addition, the catalytic reactive sites can be induced via doping, and different doping atoms always generate desperate catalytic sites [10]. In the case of graphene catalysts, the electrocatalytic reactive sites for oxygen reduction reaction (ORR) in B-doped graphene are $\mathrm{B}$ atoms [80, 81], but those of $\mathrm{N}$-doped graphene are $\mathrm{C}$ atoms next to $\mathrm{N}$ dopants [82]. In addition, improved photocatalytic performance has been obtained in B-doped and P-doped $\mathrm{g}-\mathrm{C}_{3} \mathrm{~N}_{4}$ nanosheets [83-85].
(2) Surface/interface characteristics. In general, the basal planes of most 2D nanocatalysts are inertial [76]. A typical example is TMDs. As exhibited by many experiments, doping is able to effectively activate the $\mathrm{S}$ sites on the original inertial surfaces for catalysis via introducing $\mathrm{Fe}, \mathrm{Co}$ and $\mathrm{Ni}$ atoms in TMDs [86, 87]. This phenomenon can be attributed to reduced antibonding states [88]. As reported, the $\Delta G_{\mathrm{H}^{*}}$ of Co-doped $\mathrm{MoS}_{2}$ nanosheets dropped to $0.1 \mathrm{eV}$ from the original $0.2 \mathrm{eV}$ of $\mathrm{MoS}_{2}$ nanosheets [89]. Constructing heterostructures is another significant protocol for controlling surface/ interface characteristics to enhance catalytic activities, according to the complex chemical bonds at the interfaces of disparate nanomaterials [77, 90, 91]. At the meanwhile, synergistic interactions regulate the surface/interface properties via physically adjusting confined electron transfer. For instance, Qiao's group composited g- $\mathrm{C}_{3} \mathrm{~N}_{4}$ and $\mathrm{N}$-doped graphene and realized an enhanced electrocatalytic HER, as a result of the heterostructure structures and their synergistic interactions are beneficial to the proton adsorption/reduction kinetics at the surfaces/interfaces [77]. Besides, Tu et al. observed an obvious enhanced photocatalytic simultaneous reduction-hydrolysis in a hybrid structure of $\mathrm{TiO}_{2}$-graphene nanosheets, whose $\mathrm{Ti}^{3+}$ on the surface can prevent the recombination of electronhole pairs during the production of methane $\left(\mathrm{CH}_{4}\right)$ and ethane $\left(\mathrm{C}_{2} \mathrm{H}_{6}\right)$ [92].

(3) Electronic states. For all catalytic reaction systems, electronic states determine the separation and transport of electric carriers, which will have a large impact on practical catalytic performance [34]. In general, high level of carrier transport mobility benefits to these electric carriers moving to the catalytic reactive sites. Xie and the co-workers modulated the electronic structures and raised the intrinsic conductivity of $\mathrm{MoS}_{2}$ electrocatalysts through the ways of constructing controllable disordered structures and oxygen doping, which demonstrated excellent electrocatalytic HER [93]. In a twodimensional catalyst, the electronic states of defects and their adjacent regions are often different from those of other parts without defects [94]. Take graphene as an example, density functional theory (DFT) calculations have been performed to support defects enriched at zigzag edges of nanomaterials, which exhibited distinct electronic density of states that were react actively for electrocatalytic ORR [71, 95, 96]. In addition, the electronic states of 2D nanocatalysts can be altered by lattice strain as well, which can optimize catalysis performance for further [97-99]. It was reported that lattice-strained $1 \mathrm{~T} \mathrm{WS}_{2}$ nanosheets performed enhanced 
HER, which owned crystal lattices with large deformation and $\sim 3 \%$ high local strain regions [72].

(4) Energy band structures. As generally known, the light absorption properties of 2D nanomaterials for photocatalysis are largely dependent on energy band structures [100], where photoinduced related discussions will be analyzed in the next subsection for the specificity in strategies of 2D nanomaterial-based photocatalysis. In addition to the controlling for light absorption properties, energy band structures typically regulate redox potentials to drive electric carrier dynamics for catalysis reactions including photocatalysis as well as electrocatalysis [101-103]. Additionally, the phase transition of 2D nanocatalysts can also regulate the energy band structures as well as related electronic states for controlling catalytic performance [104]. Take TMDs as example, through the approach of lithium intercalation for monolayered $\mathrm{MoS}_{2}, \mathrm{WS}_{2}$ and tungsten selenide $\left(\mathrm{WSe}_{2}\right)$, their band gap structures were regulated to improve the charge transfer kinetics, and their electronic properties exhibited metallic [105]. As a result, the electrocatalytic HER performance could be enhanced within the phase transition from $2 \mathrm{H}$ to $1 \mathrm{~T}$ [105]. In addition to the modified band gap structures according to the improved layer spacing, the oxidation states of Mo and $\mathrm{W}$ decreased with the regulation of d-band filling, on the basis of the experimental results [104].

Generally, the above-mentioned four aspects are the most common strategies for catalytic performance enhancement covering photocatalysis and electrocatalysis, where they are correlative instead of independent of each other; as a result, they are always conditioned together.

\subsection{Differences in Strategies of Photocatalysis and Electrocatalysis}

The biggest differences between photocatalysis and electrocatalysis are diverse driving ways for redox reactions that photoinduced electric carriers and external circuitinduced carriers dominate the catalytic reaction processing, respectively.

For photocatalysis, the development of light absorption catalysts is a key goal for photocatalysis [6], but conventional photocatalysts suffer from an uncontrolled extinction coefficient and severe photocorrosion during irradiation by sunlight; these factors result in poor catalytic performance and limited practical applications [106]. For example, as a representative all-organic semiconductor material, a layered $\mathrm{g}-\mathrm{C}_{3} \mathrm{~N}_{4}$ has been widely reported in catalytic-related applications [107-111]. However, pure g- $\mathrm{C}_{3} \mathrm{~N}_{4}$ nanomaterials continue to suffer from secondary pollution, limited visible light absorbance and high electron-hole recombination, which remain significant challenges for the development of highly efficient catalysis [112-116]. To meet these challenges, a variety of $\mathrm{g}-\mathrm{C}_{3} \mathrm{~N}_{4}$-based composite catalysts with core-shell structures have been reported in an effort to improve the photoresponse to visible light and carrier separation [117-120]. The core-shell structures of hybrid catalysts promote the separation of photoinduced carriers in disparate components, resulting in an enhanced photocatalytic performance. Wang et al. reported on a sol-gel synthesized few-layered g- $\mathrm{C}_{3} \mathrm{~N}_{4} @ \mathrm{TiO}_{2}$ core-shell nanocomposite catalyst for efficient visible light photocatalysis, where the layers could be finely controlled through the regulation of the colloidal suspension concentration and calcination temperature, as shown in Fig. $2 \mathrm{a}$ [121]. In this case, $\mathrm{TiO}_{2}$ makes up for the lack of light absorption and photoresponse of the layered $\mathrm{g}-\mathrm{C}_{3} \mathrm{~N}_{4}$ nanomaterials, while the generated chemical bonds of the $\mathrm{g}-\mathrm{C}_{3} \mathrm{~N}_{4}$ shell and $\mathrm{TiO}_{2}$ core benefit for photoinduced carrier separation [121]. In addition, the doping of layered $\mathrm{g}-\mathrm{C}_{3} \mathrm{~N}_{4}$ nanomaterials, as an effective modification strategy, is able to regulate the electronic structure to control the light responsive range and improve carrier separation [110, 120, 122-128], where the S, B, F and C atoms replace lattice atoms, and transitional metals are incorporated into the framework [129-135]. Xiong et al. designed a unique electronic structured K-doped $\mathrm{g}-\mathrm{C}_{3} \mathrm{~N}_{4}$ nanomaterial and achieved an excellent enhancement in photocatalytic nitric oxide (NO) removal performance, as shown in Fig. 2b [136]. According to the DFT calculations, the use of K intercalated doping with a specific structure of $\mathrm{K}$ atoms that can bridge the layers leads to a narrowing of the bandgap of $g-\mathrm{C}_{3} \mathrm{~N}_{4}$, thereby leading to decreased electronic localization, positively shifted valence band position and an enlarged $\pi$ conjugated system. As a result, the K-doped $\mathrm{g}-\mathrm{C}_{3} \mathrm{~N}_{4}$ nanomaterial provides an enhanced visible light absorbance, effective carrier separation and strong oxidizing property [136].

Take COFs as example, most photocatalysis-related research based on COFs is in the field of the reduction in carbon dioxide and production of hydrogen; however, there are few reports of the use of COFs for photocatalytic treatment of toxic organic pollutants in wastewater. In addition, the disadvantages of high cost, harsh synthesis conditions 
(a)
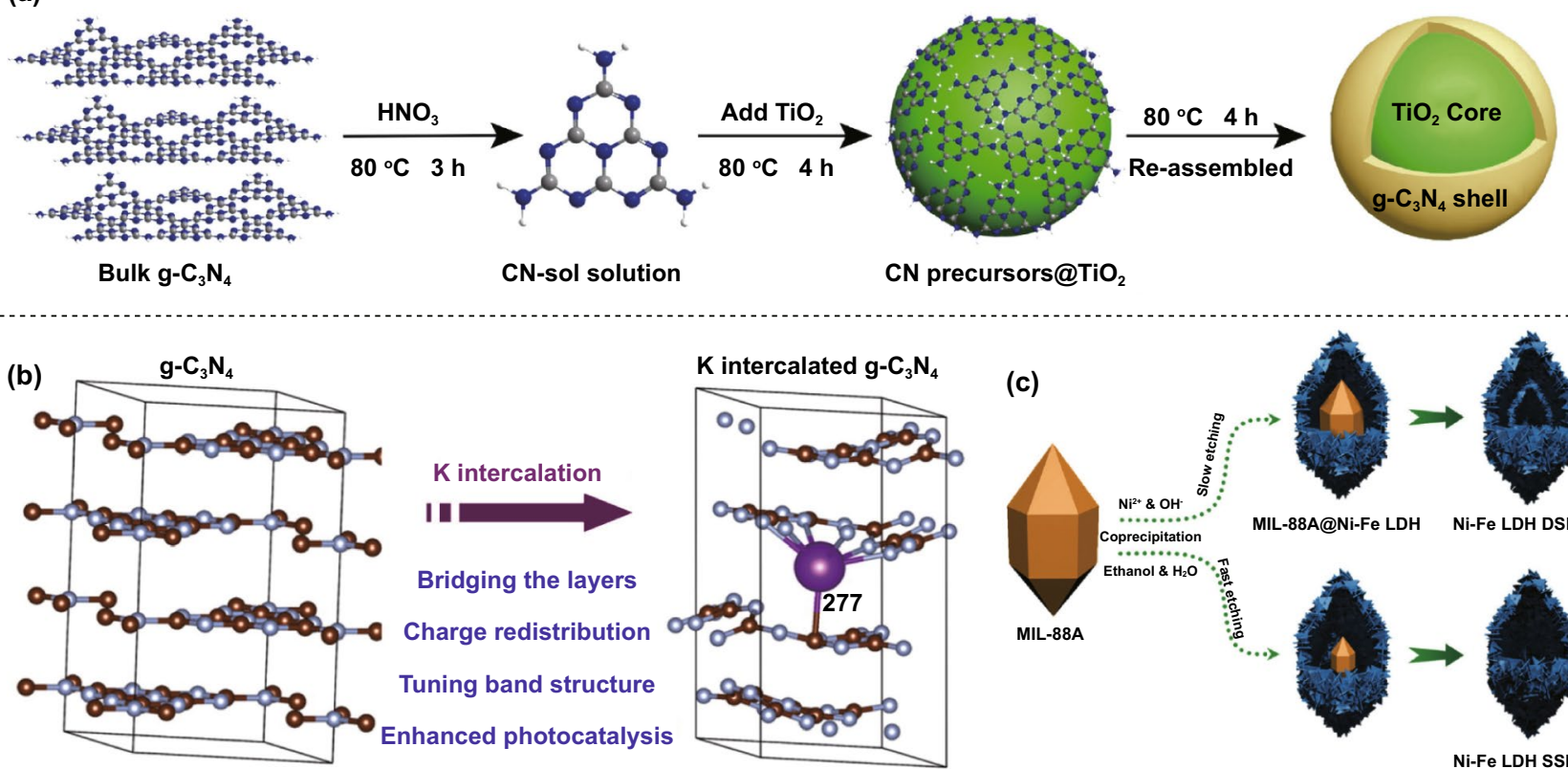

(c)
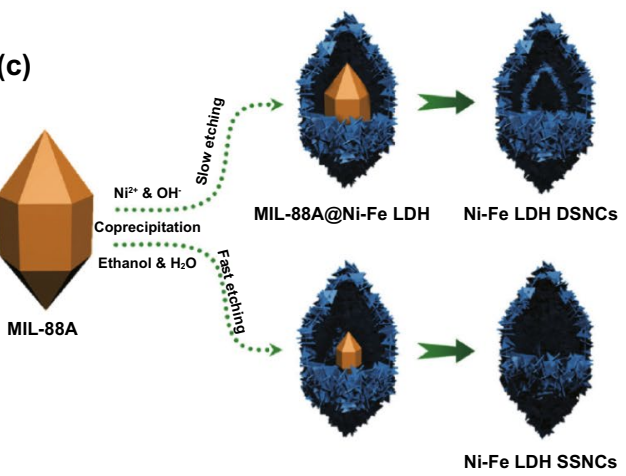

Fig. 2 Strategies for catalytic activity enhancement. a Core-shell structures of $\mathrm{g}-\mathrm{C}_{3} \mathrm{~N}_{4} @ \mathrm{TiO}_{2}$ promote carrier separation. Reproduced with permission [121]. Copyright 2018, Elsevier. b K-doped g- $\mathrm{C}_{3} \mathrm{~N}_{4}$ nanomaterials to achieve enhanced visible-light absorption, efficient carrier separation and strong oxidation capability. Reproduced with permission [136]. Copyright 2016, American Chemical Society. c Ni-Fe LDH nanocages with regulated tunable shells perform optimal chemical composition possessing large electroactive surface area. Reproduced with permission [155]. Copyright 2020, Wiley-VCH

and long reaction time make COFs less economically viable for practical applications such as environmental wastewater treatment. This is probably a result of the COFs structure being formed by covalent bonds, in contrast to metal organic frameworks (MOFs) which are formed by coordination bonds. In addition, the most significant challenges for the creation of new COFs catalysts are their low chemical stability, low catalytic efficiency and low cost-efficiency, where a series of strategies for synthesis and design approaches are needed; this includes a need for excellent chemical stability, strong catalytic activity and high cost-efficiency. As a result, COFs are often combined with other materials, such as $\mathrm{TiO}_{2}, \mathrm{MoS}_{2}$, cadmium sulfide (CdS), zinc sulfide ( $\mathrm{ZnS}$ ), cadmium selenide ( $\mathrm{CdSe})$ and graphene, to enhance visible light absorption, promote electric carrier transfer efficiency and increase specific surface area [137-140]. This is achieved by taking advantage of its large conjugated structure that is conducive to electron transport and strong visible light absorption. It is worth highlighting that another strategy for optimizing COFs is to establish favorable electron donor-acceptor characteristics, for example by using active metal nanoparticles to provide improved charge separation and a broadening of the absorption range [141-146]. As a result, a range of optimized COFs matrix composites with excellent photocatalytic activity has been reported to provide efficient treatment of organic pollutants in wastewater.

For electrocatalysis, electrochemical water splitting has promising capacity for hydrogen and oxygen production; however, the oxygen evolution reaction is limited due to a non-negligible overpotential and depressed reaction kinetics [147-149]. In general, active sites for oxygen generation are located on the catalyst surface; thus, a large surface area of the catalyst is desirable for excellent catalytic performance $[13,78,148]$. Ni-Fe-layered double hydroxides (LDHs) have been reported as an excellent oxygen evolution catalyst in an alkaline solution due to synergistic interactions between $\mathrm{Fe}$ and $\mathrm{Ni}$ [150-154]. The subtle design of nanomaterial structure can be considered to be one of the most significant strategies for catalysis reaction enhancement for electrocatalytic applications [155]. Zhang et al. synthesized Ni-Fe LDH nanocages with regulated tunable shells, and realized noble electrocatalysis for the oxygen evolution reaction, where Ni-Fe LDH materials with an optimal chemical composition possessed a large 
electroactive surface area, as shown in Fig. 2c [155]. In addition, the process of sonication can modify the LDH hydrophobic surface in a xylene solution and break the LDH into small fragments with an average lateral size that is decreased to dozens of nanometers [156].

To sum up, there are abundant strategies that have been explored and analyzed for photocatalytic and electrocatalytic activity enhancement in 2D nanomaterials, which can be generally concluded as the number of reactive sites, surface/interface characteristics, electron states and energy band structures; however, specific strategies for photocatalysis and electrocatalysis are, respectively, on the basis of light absorption and electron transfer. The above-mentioned strategies can be achieved via the approaches, such as doping, heterojunctions, phase transition and defects.

\section{Traps of Catalytic Systems in 2D Nanomaterials}

As researchers gradually in-depth research in the research field of photocatalysis and electrocatalysis, there are increasing publications focused on catalytic performance enhancement of 2D nanomaterials. Most of the catalyticrelated laboratory works, ultrahigh catalytic behaviors are desirable, but these probably seem to be a series of traps under the laboratory circumstances, due to rare 2D nanocatalyst products possessing long-term stability in efficient catalytic activity in reality. Besides, each research group draws up specific rules for their own catalytic reaction systems including the experimental parameters of additive amount, external energy input and environmental implication. The specificity of catalytic system designing is hard to avoid the yielding of a series of traps during the experimental processes according to such freedom. These traps can be considered as details that are easy to be overlooked during the catalytic reaction systems, which may lead to improved catalytic activities. In this section, we will give a brief summary for general and special traps of different catalysis systems in 2D nanomaterials, which also can be seen in the dendrogram of Fig. 3.

For general catalysis, experimental environment and 2D nanocatalyst-related issues are the most factors that are able to be possible traps in experimental catalysis systems. First, the impurities from the catalytic reaction systems are able to largely influence the final catalytic performance including the purity of 2D nanocatalysts and the contamination of glassware, especially when the reaction product yield at a relatively low level. For instance, the generation and deposition of metallic impurities from the counter electrode during the catalytic reaction may give the catalytic possibility for materials originally incapable of catalytic activity $[157,158]$. When the catalytic solutions are extremely alkaline, the generation of silicates from glassware could have an impact on the results of the catalytic experiments [159]. Second, in addition to the resulting generation of impurities from surroundings, the solution $\mathrm{pH}$ is considered as a key role in controlling protonated states of reactants; as a result, the $\mathrm{pH}$ parameters should be disclosed when writing articles. For example, the formation of bicarbonate when catalytic carbon dioxide $\left(\mathrm{CO}_{2}\right)$

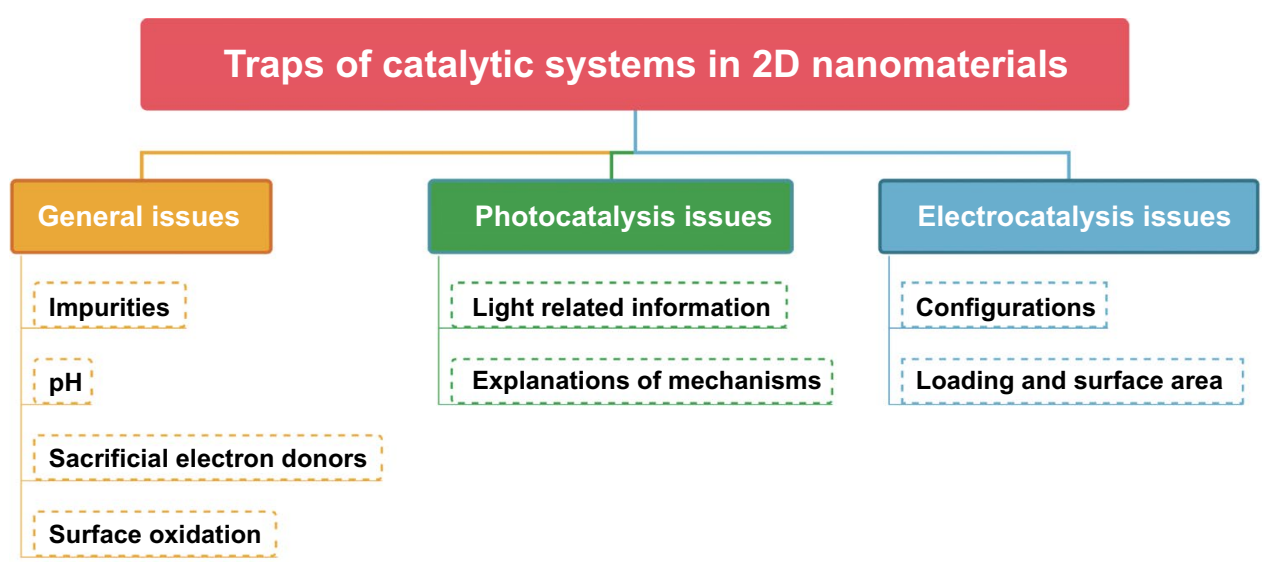

Fig. 3 A brief summary for general and special traps of different catalysis systems in 2D nanomaterials. General issues include impurities, pH, sacrificial electron donors and surface oxidation. Specific photocatalysis issues include light-related information and explanations of mechanisms. Specific electrocatalysis issues include configurations and loading and surface area 
reduction reactions can decrease the $\mathrm{pH}$ of the solutions [160]. Third, during catalytic $\mathrm{CO}_{2}$ reduction activities and hydrogen evolution, sacrificial electron donors are always added for the promotion of reductive reactions [161], but the product yields may impact when introducing sacrificial electron donors. For example, when catalytic $\mathrm{CO}_{2}$ reduction reactions processing, organic sacrificial electron donors, such as ethylene diamine tetraacetic acid (EDTA) and triethanolamine (TEOA), may be oxidized, which will further generate $\mathrm{C}_{1}$ [162]. Similarly, the oxidation of alcohols and $\mathrm{SH}^{-}$can evolute $\mathrm{H}_{2}$ during catalytic hydrogen evolution reactions [163]. Fourth, surface oxidation of 2D nanocatalysts for photocatalysis and electrocatalysis is common phenomenon, where the formation of hydroxide or metal oxide surface layer could protect the catalyst for stable catalytic activities [164]. Herein, it is of great significance to make the real substance clear through surface-sensitive techniques before and after the catalytic reactions.

For photocatalysis, the photocatalytic performance-related performances, covering the parameters of quantum yields, energy efficiencies and reaction rates, depend largely on photon flux, wavelength and scattering [165]. Therefore, these light-related information as well as nanocatalyst's light absorption are key factors for photocatalysis that must be considered and mentioned in the manuscript, but till now less photocatalytic-related papers involve the information. This undoubtedly makes it difficult for other researchers to repeat and improve the experiments. In addition, the explanations of mechanism for photocatalytic systems are limited in hydroxyl radical-mediated reactions and interfacial electron transfer processes. However, simple assumption according to previous work is far from enough to prove the photocatalytic mechanisms, due to every photocatalytic reaction system is unique [166]. As a consequence, more analysis is needed to rule out the irrelevant explanations for specific photocatalytic reaction systems.

When conducting electrocatalytic experiments, specific electrochemical configurations are of great importance, such as three-electrode or two-electrode configurations. For three-electrode configurations, there will be large deviation for electrocatalytic products of counter electrode compartment, if the counter electrode's potential does not keep a set level. For electrocatalytic reaction systems possessing the separating membrane between different compartments, the drop of voltage for membrane structure should be considered rigorously [167]. Similarly, when reporting overpotential, it is important to carefully consider relevant configuration issues [168]. In addition to configuration issues, the loading and surface area of 2D nanocatalysts also exhibit impact on the specific electrocatalytic overpotential; as a result, the information of 2D nanocatalyst loading and morphologicalbased surface area must be provided in the manuscript, but a great deal of literatures are not available [169].

In this section, we have discussed the general and special traps of different catalysis systems in 2D nanomaterials. General issues include impurities, $\mathrm{pH}$, sacrificial electron donors and surface oxidation. Specific photocatalysis issues include light-related information and explanations of mechanisms. Specific electrocatalysis issues include configurations and loading and surface area.

\section{D Nanocatalysts}

In Sect. 4, we will briefly introduce typical 2D nanocatalysts that have long been considered research hotspots for general catalytic applications, through discussing their classification, structures, synthesis approaches and characterizations in turn as the following subsections.

\subsection{Classification of 2D Nanocatalysts}

The development of atomically thin 2D graphene nanomaterials has propelled progress in related ultrathin 2D nanomaterials [12]. In general, most 2D nanomaterials can be sketchily classified into layered materials [44], where van der Waals interactions between layers make layer stacking, and continuous atom layers within layers are typically strong chemical bonded [170]. Typical 2D layered nanomaterials for catalysis include graphene, graphitic carbon nitride, a family of mono-elemental compounds, TMDs, COFs, metal carbides and nitrides (MXenes), LDHs, bismuth-based layered compounds, hexagonal boron nitride (h-BN) MOFs and 2D metal nanomaterials, etc.

The structure of graphite was determined with the advent of single-crystal X-ray crystallography, where graphene is generally considered as an atomically thin single-layer graphite crystal [171]. Atomically thin graphene nanomaterials are promising materials with a performance that can exceed conventional semiconductors for catalytic applications $[12,37]$. However, graphene nanomaterials are a form 
of zero band gap semi-metal [172]; therefore, they are usually considered as a co-catalyst or an effective catalyst support, rather than a catalyst directly [173].

One of the most well-known structural graphene-like 2D nanomaterials is $\mathrm{g}_{-} \mathrm{C}_{3} \mathrm{~N}_{4}$ [107]. Generally, g- $\mathrm{C}_{3} \mathrm{~N}_{4}$ nanomaterials are able to act as a potential catalyst in numerous redox reactions due to their chemical inertness under strong acid or alkali environments [64]. However, challenges such as high electric carrier recombination rate, low specific area and poor mass transfer can restrict the efficiency of catalysis, where approaches such as heterojunction coupling, surface defect engineering and element doping have been considered to address this deficiency [124].

Pnictogens are mono-elementals of group VA that exhibit a high energy and power density and can be produced by stacking layered materials of various characteristics to create a heterostructure to combine the superior aspects of each material [174]. As a typical family of mono-elemental compounds, an ultrathin 2D structure of BP in the orthorhombic phase was first synthesized in 1914 [175]. Compared to graphite, black phosphorus can enhance the specific capacity from 372 to $4200 \mathrm{mAh} \mathrm{g}^{-1}$, with a reversible reaction with $\mathrm{Li}$ and $\mathrm{Na}$ [176-179]. Moreover, their thermodynamically stable properties for electronic applications enable operation at extreme temperatures and at humidity in air, which results in efficient and stable catalytic reactions [180-184].

TMDs generally consist of chalcogen atom layers, with a transition metal atom interlayer [185, 186]. Changing the number of layers in the crystal provides an opportunity to regulate the band gap of TMDs. $\mathrm{MoS}_{2}$-based nanomaterials, as a typical TMD material, exhibit unique lattice vibration properties, high catalytic activity, low cost and natural abundance [63]. To date, 2D layered $\mathrm{MoS}_{2}$ nanomaterials have demonstrated significant potential to replace graphene nanomaterials in a variety of applications due to their unique characteristics. Zhang et al. utilized $\mathrm{MoS}_{2}$ to achieve outstanding catalytic properties for the $\mathrm{N}_{2}$ reduction, compared to other catalysts reported under the same circumstances, where the Faradaic efficiency and the $\mathrm{NH}_{3}$ yield rate reached high levels of $1.17 \%$ and $8.08 \times 10^{-11} \mathrm{~mol} \mathrm{~s}^{-1} \mathrm{~cm}^{-2}$, respectively [187].

COFs are formed from organic ligands through reversible covalent bonds and are considered as advanced crystalline porous materials. In 2005, Yaghi and co-workers provided the first demonstration of connecting small symmetric organic structural units to a covalent organic skeleton of a porous crystal using the principle of dynamic covalent chemistry [188]. Tan's group explored a green and facile approach for creating a 2D heterogeneous P6-Au-COF hybrid nanomaterial that was formed using a $\mathrm{COF}$ and pillar[6]arene reduced $\mathrm{Au}$ nanoparticles (P6-Au), which showed high catalytic performance for the reduction in nitrophenol isomers [189].

Graphene-like MXenes have been synthesized from stacks of scrolls and sheets, including mono-transitionmetal MXenes and double-transition-metal MXenes [190-198]. Monolayered MXenes show metallic properties due to their high electron state concentration near the Fermi level [192, 199-202]. A high electron state concentration near the Fermi level indicates that MXenes are potential layered materials for catalytic applications [191]. MXenes possess excellent electronic conductivity, high elastic moduli and good hydrophilic properties that have been exploited in a variety of applications such as hybrid electrochemical supercapacitors and Li-ion battery anodes [194, 200, 203-212].

LDHs are constructed using brucite-like host layers and interlayered structural water with positive charges and negatively charged anions, respectively [213, 214]. LDHs, especially those that contain transition metals, are widely reported to be a promising catalyst with a high catalytic activity for applications related to the generation of oxygen and hydrogen [215]. Zhang's group reported on ultrathin $\mathrm{NiFe}-\mathrm{LDH}$ nanosheets with a $0.6 \mathrm{~nm}$ thickness and achieved an overpotential of $254 \mathrm{mV}$ for the electrocatalytic water splitting reaction, and demonstrated superior charge transfer properties [216].

Bismuth, as an environmentally friendly metal, possess a wide range of interesting features for a range of applications, including catalysis [217-221]. Bismuth-based 2D layered nanomaterials have been reported to demonstrate high performance for energy conversion and storage devices [222-227]. The band gap can be manipulated from $0.3-3.6 \mathrm{eV}$, by introducing a variety of cations and anions into the intrinsic structure, whose corresponding light response spans the ultraviolet to near infrared $[65,66]$. Besides, the effective mass and mobility of photoexcited electric carriers are restricted and improved, which is beneficial to applications such as optoelectronic energy conversion, photodetection and photochemical catalysis [228, 229].

The h-BN is a hexagonal crystal system with a graphitelike hierarchical structure. It has been utilized as a catalyst 
carrier or catalyst as a result of its high temperature resistance, high thermal conductivity of $\sim 390 \mathrm{~W} \mathrm{~m}^{-1} \mathrm{~K}^{-1}$, extremely stable chemical properties, strong acid corrosion resistance, and high electrical insulation. Nevertheless, due to its low electrical conductivity, attempts have been made to functionalize the h-BN monolayer by combination with electrically conductive materials such as reduced graphene oxide (rGO) [230, 231] and carbon nanotubes (CNTs) [232], which can expand its range of applications [233].

Yaghi et al. reported a coordination compound which was synthesized from rigid organic ligands and a transition metal with a 2D structure, as a MOF [234]. MOFs exhibit a single-layered lamellar structure which is one-atom thick, resulting in high aspect ratio and the possibility of post-synthetic modification that can help realize tailor-made pores for selective adsorption and catalysis and the incorporation functional groups into the MOFs $[235,236]$. Since transition metals represent a large proportion of the MOF, it is beneficial to provide large pore dimensions, large surface area and a versatility of the type of MOF structure formed.

2D metal nanomaterials, especially noble metals [237], are synthesized as the forms of nanosheets [238-240], nanodisks [241], nanoplates [242, 243], nanoribbons [244], nanorings [245] and nanobelts [246], etc. Due to the interesting electronic and structural properties, 2D metal nanomaterials have been applied in a variety of catalysis [71, 247, 248]. Huang et al. reported 2D Pd nanosheets performed large enhancement in electrocatalytic formic acid oxidation reaction (FOR) in comparison with commercial Pd black [238], attributed to abundant active sites on the catalyst surface [247].

\subsection{Structures of 2D Nanocatalysts}

In the last subsection, we have discussed the classification of 2D nanomaterials. These 2D nanomaterials possess distinct crystal phases related to atomic coordination, atomic arrangement and layered stacking [97, 249, 250], which can largely regulate the properties and catalytic activities [105, 251-253]. Here, we will discuss the structures of the above-mentioned 2D nanocatalysts, where a range of $2 \mathrm{D}$ catalytic nanomaterials with distinct structures is highlighted in Fig. 4.

Graphene comprises hexagonal or honeycomb-like geometry carbon atoms in $s p^{2}$-hybridized form, where every carbon atom connects with the adjacent three atoms via $\sigma$-bond covalently bonding [254].

The planar structure of $\mathrm{g}-\mathrm{C}_{3} \mathrm{~N}_{4}$ is specifically different from that of graphene, where carbon and nitrogen atoms constitute $\mathrm{N}$-substituted graphite frameworks in $s p^{2}$-hybridized form [255-257]. In general, $\mathrm{g}-\mathrm{C}_{3} \mathrm{~N}_{4}$ possesses two typical structures that are formed by tri-s-triazine units and $s$-triazine units $[255,258,259]$. For $g-\mathrm{C}_{3} \mathrm{~N}_{4}$ with tri-s-triazine units, at a temperature of $900 \mathrm{~K}$ in vacuum, the structure of the $\mathrm{g}_{-} \mathrm{C}_{3} \mathrm{~N}_{4}$ monolayer becomes disordered, where the hydrogen bonds within $\mathrm{NH} / \mathrm{NH}_{2}$ groups are broken, resulting in $\mathrm{NH} / \mathrm{NH}_{2}$ groups twisting outward, as seen in Fig. $4 \mathrm{a}$ [260]. Such amorphous $\mathrm{g}^{-} \mathrm{C}_{3} \mathrm{~N}_{4}$ nanomaterials can achieve enhanced photocatalysis for hydrogen generation in contrast to the crystalline form of $\mathrm{g}-\mathrm{C}_{3} \mathrm{~N}_{4}$ when illuminated by visible light [260].

The layered puckered honeycomb structural BP exhibits an orthorhombic crystal and a space group of Cmca. Each $\mathrm{P}$ atom connects with the adjacent three $\mathrm{P}$ atoms, in which three locate at the same plane, but the rest one atom locates at another plane [261,262]. Figure 4b illustrates the typical structure of BP from a top view [263].

Monolayered TMDs stack together to form layered TMDs via van der Waals interactions in general. Individual monolayered TMD is made of one sandwiched transition metal atomic layer and two chalcogen atomic layers [24]. The structure of a TMD is shown in Fig. $4 \mathrm{c}$ which indicates both the top and side views, where the chalcogen atoms and the metal atoms are bonded covalently with trigonal prismatic coordination [264].

Periodic porous COFs are orderly formed by organic building block units with covalent connection [265, 266]. A typical structure of a COF is illustrated in Fig. 4d [189], which has the advantages of a high level of inherent porosity, adjustable aperture, good conjugation structure, large surface area, crystallizability, no secondary pollution and wide visible light response range.

Layered MXenes $\left(M_{n+1} X_{n} T_{x}\right)$ can be gained via selective etching treatment for A-group elements from parent layered ternary carbides $\left(M_{n+1} A X_{n}, M A X\right)$ [194-198], where MAX possesses hexagonal structure with a space group of $\mathrm{PG}_{3} / \mathrm{mmc}$ [267]. Geng et al. achieved notable catalytic hydrogen evolution reactions using a $\mathrm{Mo}_{2} \mathrm{C}$-on-graphene MXene heterostructure, where the crystal structure of $\mathrm{Mo}_{2} \mathrm{C}$ can be seen in Fig. 4e [191]. 


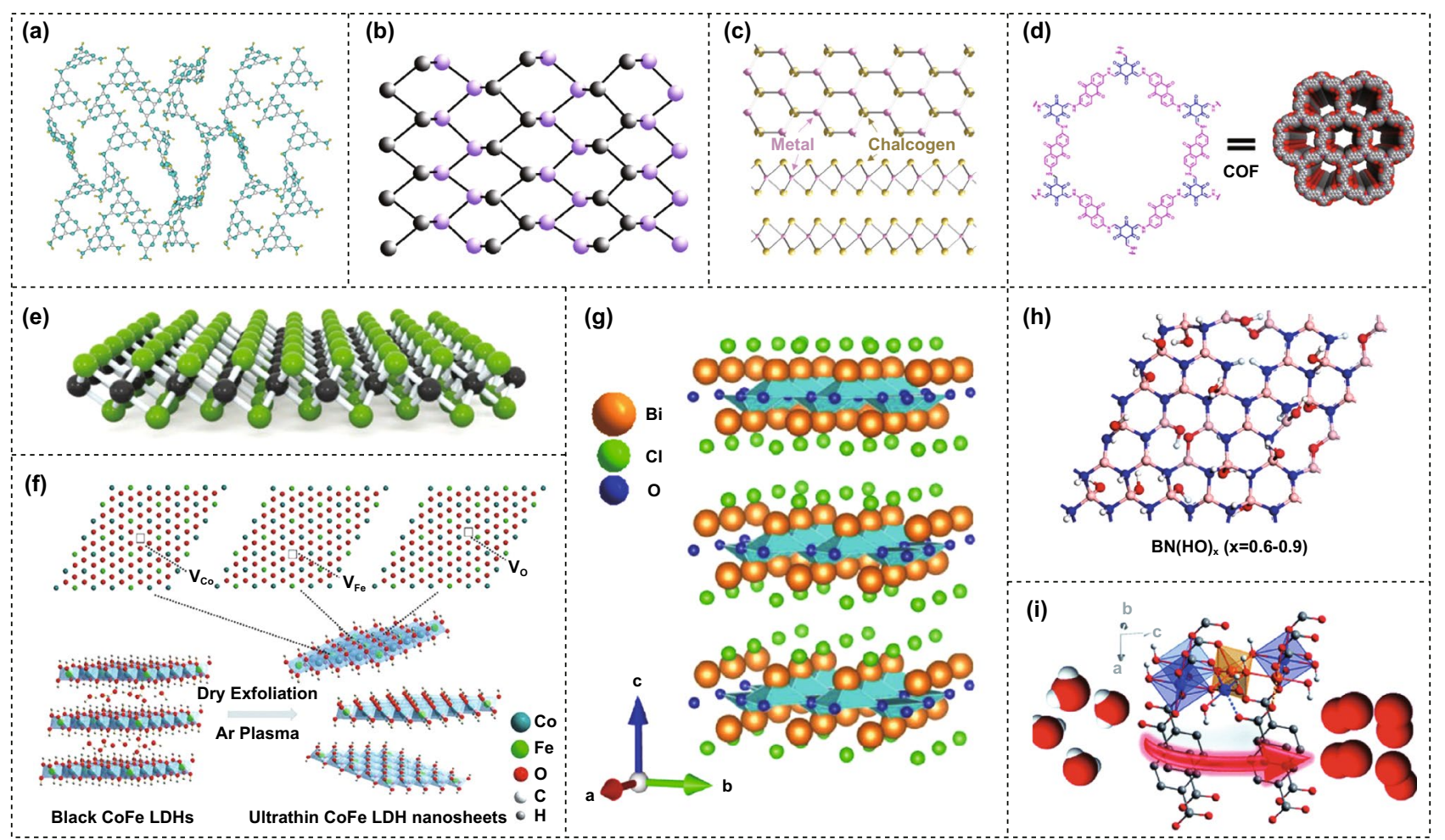

Fig. 4 Example 2D nanomaterial structures. a Graphene and graphitic carbon nitride. Reproduced with permission [260]. Copyright 2015, Wiley-VCH. b Family of mono-elemental compounds. Reproduced with permission [263]. Copyright 2018, Springer Nature. c TMD. Reproduced with permission [264]. Copyright 2017, American Chemical Society. d COF. Reproduced with permission [189]. Copyright 2020, IOP Publishing Ltd. e MXene. Reproduced with permission [191]. Copyright 2017, Wiley-VCH. f LDHs. Reproduced with permission [271]. Copyright 2017, Wiley-VCH. g BiOX. Reproduced with permission [275]. Copyright 2020, American Chemical Society. h h-BN. Reproduced with permission [276]. Copyright 2014, American Chemical Society. i MOF. Reproduced with permission [277]. Copyright 2020, Royal Society of Chemistry

The general formula of LDHs is $\left[\mathrm{M}_{1-\mathrm{x}}{ }^{2+} \mathrm{M}_{\mathrm{x}}{ }^{3+}(\mathrm{OH})_{2}\right]$ $\left(\mathrm{A}^{\mathrm{n}-}\right)_{\mathrm{z} / \mathrm{n}} \cdot \mathrm{yH}_{2} \mathrm{O}$, where the marks of $\mathrm{M}^{2+}, \mathrm{M}^{3+}$ and $\mathrm{A}^{\mathrm{n}-}$ represent divalent metal cation, trivalent metal cation and interlayer anion, respectively. $\mathrm{A}^{\mathrm{n}-}$ locates in the hydrated interlayer gap, while $\mathrm{M}^{2+}$ and $\mathrm{M}^{3+}$ locate octahedral holes in the brucite-like layer [268-270]. Wang et al. synthesized ultrathin $\mathrm{CoFe} \mathrm{LDH}$ nanosheets for use as a highly efficient oxygen evolution electrocatalyst, where the Ar plasma exfoliation fabrication process and the variety of structures formed can be seen in Fig. 4f [271].

Bismuth-based 2D layered nanomaterials achieve highdispersion band due to $s$ - $p$ hybridization and anisotropic $p$, and thus, the photogenerated carriers exhibit low effective mass and electron-hole pairs with high mobility [272-274]. In particular, bismuth oxychloride $(\mathrm{BiOCl})$ is a typical bismuth-based layered nanomaterial with a tetragonal structure and a $\mathrm{P} 4 / \mathrm{nmm}$ space group. The electronic properties and lattice dynamics of this material have been reported, and the layered crystal structure is illustrated in Fig. $4 \mathrm{~g}$, where the $\mathrm{Bi}$ and $\mathrm{O}$ atom layers are sandwiched between the $\mathrm{Cl}$ atom layers [275].

$\mathrm{H}-\mathrm{BN}$ is a hexagonal crystal system with a graphite-like hierarchical structure, whose crystal structure can be seen in Fig. 4h [276]. Normally, h-BN nanosheets are formed from $s p^{2}$ hybridized $\mathrm{B}$ atoms, along with $\mathrm{N}$ atoms that are regularly arranged in a hexagonal ring network between the individual layers [276]. In a similar manner to most layered nanomaterials, the $\mathrm{B}$ and $\mathrm{N}$ atoms are tightly covalently bonded within the layer planes. Moreover, the weak van der Waals interactions of the interlayer bonding are beneficial for material exfoliation to create ultrathin nanosheets [12].

In general, MOFs can be considered as the metal-organic skeleton materials that are self-assembled via coordination bonds between metal ions/clusters and organic ligands. The organic ligands in MOFs are called the linkers, and the metal ions or clusters are called the nodes, which are then 
self-assembled into coordination compounds with periodic structures. Li and the co-workers constructed 2D layered MOFs from LDHs via a facile ligand-assisted procedure, where the material exhibited a superior performance for water oxidation, which are illustrated in Fig. 4i [277]. However, the electrical conductivity of 2D MOFs is relatively poor, below $10^{-14} \mathrm{~S} \mathrm{~cm}^{-1}$ [278, 279], as a result of the internal porosity due the stacking of several atomic layers [280]. In addition, 2D MOFs suffer from a high sensitivity to humidity and structural instability; this is due to the weak coordination bonds that are located between the metal nodes and linkers [280, 281].

\subsection{Synthesis of 2D Nanocatalysts}

The top-down and bottom-up methods are regarded as the two primary procedures for the synthesis of 2D layered nanomaterials synthesis, and the general approaches are summarized in Fig. 5. These approaches include a variety of common methods which are based on top-down and bottomup methods.

Top-down synthesis is generally considered as a demixing processes of layered bulk materials by the external forces [282]. The key to the approach is the breaking of weak interlayered van der Waals interactions and achieve the cleavage of bonds along the layer plane to obtain 2D ultrathin nanosheets [280]. There are a number of approaches for synthesizing 2D nanomaterials via top-down methodologies, these include liquid/gas exfoliation [283], mechanical cleavage [284, 285], shaking treatment [286], wet ball milling [287, 288], sonication [216, 289] and chemical etching
[287]. In the following discussion, we will discuss common approaches for top-down 2D layered nanomaterial synthesis.

Liquid exfoliation methods have been considered as one of the most popular ways for the preparation of multiple 2D layered nanomaterials, whose synthesis mechanism is based on weakening interlayer interactions by introducing guest molecules to enlarge the spacing of interlayers, and interfacial debonding for the formation of a steady sol via ultrasonic processing [290]. Zhang's group utilized N-methyl2-pyrrolidone solutions for exfoliation of few-layer black phosphorus nanosheets to synthesize 2D layered electrocatalysts and achieved high electrocatalytic performance for oxygen generation applications [24, 226].

The mechanical cleavage method is also a common procedure for the preparation of multiple 2D layered nanomaterials. Subbiah and Jayasena utilized an ultrasharp diamond wedge that was assisted by ultrasonic oscillations to exfoliate 2D graphene layers with an approximate area of $300 \times 900 \mu \mathrm{m}^{2}$ [285]. The well-known Scotch tape method, regarded as one of most common mechanical exfoliation methods [24], led to the discovery of graphene [291] and is able to prepare high-quality 2D monolayered nanomaterials with desirable performance. For example, Fuhrer's group demonstrated a large single crystal of diindium triselenide $\left(\mathrm{In}_{2} \mathrm{Se}_{3}\right)$ exhibits strong optical properties, which was formed by exfoliation in a similar way to graphite using sticky tape (3 M Scotch) with a thickness of $100 \mathrm{~nm}$ [284].

The wet ball milling approach is a method of grinding materials, solvents and zirconia spheres into nanometer slurry at a certain ratio. Yang's group reported on the synthesis of ultrathin layered MOFs with high crystallinity,

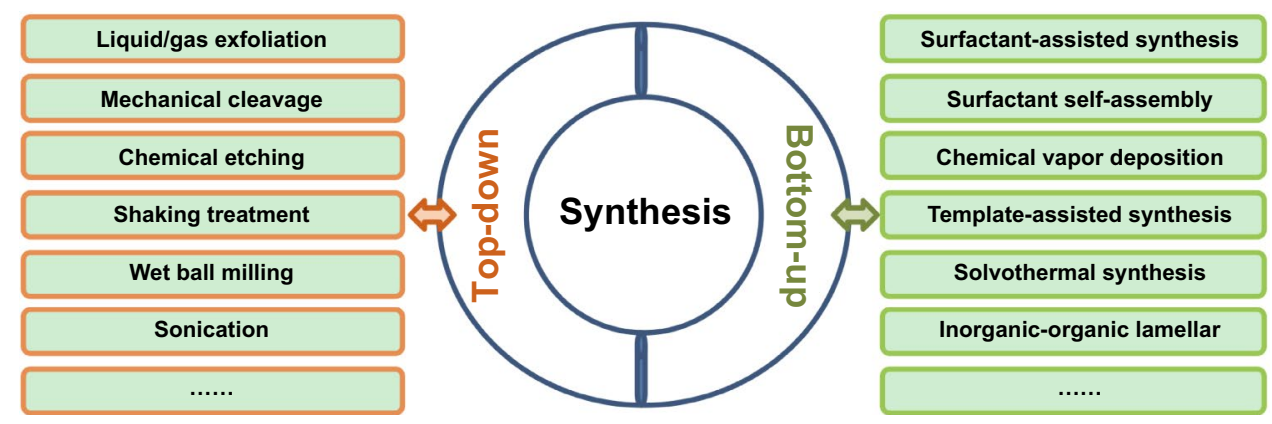

Fig. 5 Common synthesis procedures for 2D nanomaterial based on top-down and bottom-up methods. Top-down methods include liquid/ gas exfoliation, mechanical cleavage, chemical etching, shaking treatment, wet ball milling, and sonication. Bottom-up approaches include surfactant-assisted synthesis, surfactant self-assembly, chemical vapor deposition, template-assisted synthesis, solvothermal synthesis and inorganic-organic lamellar 
large lateral area and $1 \mathrm{~nm}$ thickness, via a wet ball milling and ultrasonic exfoliation procedure, where the rotation speed was at a low level of $60 \mathrm{rpm}$, and propanol and methanol were used for ultrasonication exfoliation [288].

Although top-down methods benefit the preparation of 2D nanomaterials with exceptional properties using a straightforward approach to provide a low cost product [286], these methods continue to exhibit limitations which limit the use of 2D nanomaterials for practical applications. These include the formation of unstable nanosheets, uncontrollable layer numbers, poor homogeneity, being limited to layered materials, low product yield and the stripped nanosheets often break up and restack. Hence, we believe basic research of top-down 2D nanomaterial synthesis in needed, where progress on strategies and development will expectedly improve more effort [292], such as intercalation-assisted expansion and exfoliation [293-296], and exfoliation of layer materials containing ions or molecules between the layers [192, 293].

The bottom-up synthesis for 2D layered nanomaterials can be generally considered on the basis of the anisotropic assembly of small molecules with growth limitations in vertical directions [297]. The bottom-up wet chemical synthesis approaches are easier to realize for large-scale production and provides a more controllable synthesis of $2 \mathrm{D}$ nanomaterials. These include surfactant-assisted synthesis [298], surfactant self-assembly [299], chemical vapor deposition [300-303], template-assisted synthesis [304], inorganic-organic lamellar [305] and solvothermal synthesis [306, 307]. The following discussion will discuss typical methods for 2D nanomaterials synthesized using bottom-up methods.

Lang and co-workers synthesized atomic layered binary MOF nanosheets via a bottom-up solvothermal method and achieved excellent electrocatalysis for oxygen generation with a solvent based on a N,N-dimethylacetamide solution [307]. Chemical vapor deposition is a popular approach for large-scale $2 \mathrm{D}$ material production with promising superiority in a controllable size and thickness of material for practical applications [308]. Ji's group present a chemical vapor deposition-based approach for the synthesis of 2D black phosphorus, with average areas generally over 3 $\mu \mathrm{m}^{2}$. Song's work demonstrated chemical vapor deposition growth of high-quality h-BN nanomaterials with a thickness that typically ranged from two to five atomic layers. In this work, ammonia borane was used as the precursor for the BN, followed by a gas flow of $\mathrm{Ar} / \mathrm{H}_{2}$ at a temperature of around $1000{ }^{\circ} \mathrm{Cs}$, with a typical growth time of 30-60 $\mathrm{min}$ [300].

However, traditional bottom-up synthesis strategies often require the aid of substrate materials and surfactants. It is therefore difficult to prepare dispersed 2D nanomaterials, and the residual surfactants in the products are difficult to remove, which can limit their applications.

\subsection{Characterization of 2D Nanocatalysts}

In the above subsections, we have discussed the classification, structure, synthesis of 2D nanocatalysts. In addition to these topics, the advances in characterization technologies propel the rapid development of 2D nanomaterials for related application in catalysis as well [44, 309]. So far, there have been a series of sophisticated characterization technologies for 2D nanomaterials, including multiple optical, electron and probe microscopies and various spectroscopies, that can uncover material information of morphologies, defects, crystal phase, electron density of states and so on [310-313]. In this subsection, we will briefly introduce several typical characterization technologies for distinguishing 2D nanomaterials.

The optical microscopy (OM) can rapidly provide the location and morphology information of materials [314]. By the utilization of OM, Chiu et al. demonstrated that the stacking layers of $\mathrm{MoS}_{2} / \mathrm{WSe}_{2}$ heterostructures exhibited a clear distinction with color contrast [315]. However, more accurate value of material morphology information needs much preciser characterization such as electron and probe microscopies. Scanning electron microscopy (SEM) is the most common technique for structure, topology and morphology characterization of nanocatalysts, whose resolution generally achieves several nanometers [316]. Transmission electron microscopy (TEM) is another strong technique for morphology, crystallinity and phase characterization of nanocatalysts; besides, it can always be combined with selected area electron diffraction (SAED) patterns to get more crystallinity information [317]. Jung et al. fabricated a $\mathrm{CO}_{2}$ reduction composite photocatalyst $\mathrm{TiO}_{2}$-graphene- $\mathrm{MoS}_{2}$ and observed the composite structure and morphology through SEM, the crystallinity and phase information through TEM, respectively [318]. More accurate thickness information and electronic properties of 2D nanocatalysts can be obtained by scanning probe microscopy 
(SPM) [319], such as atomic force microscopy (AFM) [320], scanning tunneling microscopy (STM) [76], kelvin probe force microscopy (KPFM) [321] and electrostatic force microscopy (EFM) [322]. Heath's group utilized monolayered graphene to visualize water adlayer on mica with the average height of $0.37 \pm 0.02 \mathrm{~nm}$ [323]. Jaramillo et al. controlled different active sites of $\mathrm{MoS}_{2}$ electrocatalysts, and identified these active sites via STM; as a result, the HER electrocatalytic activity showed a linear relation with the amount of catalytic active sites [76]. Additionally, EFM was used to analyze the electrostatic screening effects of $\mathrm{MoS}_{2}$ atomically thin layers [322].

Apart from advanced microscopies, various sophisticated nondestructive spectroscopies are also used for characterization of 2D nanomaterials, including Raman spectroscopy [324], X-ray photoelectron spectroscopy (XPS) [72] and X-ray absorption spectroscopy (XAS) [325]. Raman spectroscopy provides spatial resolution and high spectral in the electronic and structural information of 2D nanomaterials [326]. XPS is able to distinguish different crystal phases within 2D nanocatalysts [327]. Voiry et al. calculated quantitatively each phase concentration of $\mathrm{WS}_{2}$ nanosheets with the help of XPS [72]. XAS can characterize the atomic-scale structural information, including species of the atoms, coordination chemistry and oxidation states [328]. Sun et al. proposed a pits-confined $\mathrm{CeO}_{2}$ nanosheet platform for catalytic $\mathrm{CO}$ oxidation evaluation at a variety of active catalytic centers, where it indicated by XAS that the average coordination number of pit-surrounding cerium sites was 4.6 when the artificial $\mathrm{CeO}_{2}$ nanosheets with $20 \%$ pits occupancy [325].

In Sect. 4, we have overviewed the classification, structure, synthesis and characterization of 2D nanocatalysts. The classification and structure have been discussed in a range of 2D nanocatalysts including graphene, graphitic carbon nitride, a family of mono-elemental compounds, TMDs, COFs, MXenes, LDHs, bismuth-based layered compounds, h-BN, MOFs and 2D metal nanomaterials. The common synthesis procedures for $2 \mathrm{D}$ nanomaterial are based on top-down and bottom-up methods. Topdown methods include liquid/gas exfoliation, mechanical cleavage, chemical etching, shaking treatment, wet ball milling, and sonication. Bottom-up approaches include surfactant-assisted synthesis, surfactant self-assembly, chemical vapor deposition, template-assisted synthesis, solvothermal synthesis and inorganic-organic lamellar.
In addition, we have introduced a series of typical characterization technologies for 2D nanomaterials covering the microscopies of OM, SEM, TEM, SPM and the spectroscopies of Raman, XPS, XAS.

\section{Catalytic Applications of 2D Nanomaterials}

In this section, we provide a discussion on the photocatalytic and electrocatalytic applications of 2D nanomaterials based on recent publications, which are mainly focused on environmental treatment and biochemical technologies including dye degradation, elimination of toxicant, HER, oxygen evolution reaction (OER), carbon dioxide reduction reaction $\left(\mathrm{CO}_{2} \mathrm{RR}\right)$ and cancer therapy. In addition, Table 2 summarizes the variety of $2 \mathrm{D}$ nanomaterialbased electrocatalysts applied for specific electrocatalytic reactions for practical applications, along with synthesis methods, applied conditions, detailed electrocatalytic performance and basic catalysis mechanisms.

Today, we all use a wide variety of medicines and personal care products, leading to irreversible damage as they enter the ecosystem [329]. Antibiotics and their metabolites have potential toxicological risks with regard to nonresistant microorganisms, phytoplankton, fish and other aquatic organisms that may disrupt the aquatic food chain [330]. They are difficult to fully degrade by traditional biological processes, and their toxicity may be further amplified as they accumulate in our aquatic ecosystems [331]. Therefore, there is a need to explore advanced technologies that are able to destroy organic compounds in aquatic environments. Here, we will discuss the recently reported catalytic related applications in environmental treatment and biochemical technologies using 2D nanomaterials.

\subsection{Dye Degradation}

With regard to dye degradation, it should be emphasized that 2D nanomaterials are suited for oxidative dye photodegradation reactions since their small kinetic barriers and optimal thickness provide a high surface area [39]. For example, Zhang and co-workers synthesized highly crystalline $\mathrm{BiOCl}$ single-crystalline nanosheets through a hydrothermal method [332]. Figure 6a demonstrates 
Table 2 Overview of range of 2D nanomaterials for electrocatalytic applications

\begin{tabular}{|c|c|c|c|c|c|c|}
\hline 2D nanomaterials & Synthesis & Application & Conditions & Performance or parameter & $\begin{array}{l}\text { Activity origin or } \\
\text { mechanism }\end{array}$ & Refs \\
\hline Double-gyroid $\mathrm{MoS}_{2}$ & $\begin{array}{l}\text { Electrodeposition, } \\
\text { followed by sulfidiza- } \\
\text { tion }\end{array}$ & HER & Acidic medium & $\begin{array}{l}\text { Overpotential = 150- } \\
200 \mathrm{mV} \\
\text { Tafel slope }=50 \mathrm{mV} \\
\text { decade }^{-1}\end{array}$ & $\begin{array}{l}\text { Surface embellish- } \\
\text { ment for edge site } \\
\text { exposure }\end{array}$ & {$[35]$} \\
\hline $\mathrm{Co}_{3} \mathrm{~S}_{4}$ & Physical etching & HER & Alkaline medium & $\begin{array}{l}\eta_{10}=63 \mathrm{mV} \\
\text { Tafel slope }=58 \mathrm{mV} \\
\text { decade }^{-1}\end{array}$ & $\begin{array}{l}\text { Abundant sulfur vacan- } \\
\text { cies }\end{array}$ & {$[340]$} \\
\hline $\mathrm{Co}-\mathrm{N}-\mathrm{GA}$ & Solvothermal & HER & Acidic medium & $\begin{array}{l}\text { Onset }=0 \mathrm{~V}, \\
\eta_{10}=46 \mathrm{mV} \\
\text { Tafel slope }=33 \mathrm{mV} \\
\text { decade }^{-1}\end{array}$ & $\begin{array}{l}\text { Synergetic effect of } \\
\text { N-doped carbon and } \\
\text { inner metal Co }\end{array}$ & [306] \\
\hline $\mathrm{MoS}_{2}$ & Hydrothermal & HER & Acidic medium & $\begin{array}{l}\prod_{-200}=198 \mathrm{mV} \\
\text { Tafel slope }=36 \mathrm{mV} \\
\text { decade }^{-1}\end{array}$ & $\begin{array}{l}\text { Facilitated ion dif- } \\
\text { fusion by channel } \\
\text { engineering }\end{array}$ & [36] \\
\hline $\mathrm{WSe}_{2}$ & Hydrothermal & HER & Acidic medium & $\begin{array}{l}\text { Onset }=150 \mathrm{mV} \\
\text { Tafel slope }=78 \mathrm{mV} \\
\text { decade }^{-1}\end{array}$ & $\begin{array}{l}\text { Many exposed edge } \\
\text { sites can provide } \\
\text { abundant active reac- } \\
\text { tion sites }\end{array}$ & [186] \\
\hline $\mathrm{Mo}_{2} \mathrm{CT}_{\mathrm{x}}$ & $\begin{array}{l}\text { Ball milling, HF etch- } \\
\text { ing }\end{array}$ & HER & Acidic medium & $\begin{array}{l}\eta_{10}=189 \mathrm{mV} \\
\text { Tafel slope }=70 \mathrm{mV} \\
\text { decade }^{-1}\end{array}$ & $\begin{array}{l}T_{x} \text { as surface func- } \\
\text { tional groups }\end{array}$ & [287] \\
\hline NiFe-LDH & Ultrasonication & OER & Alkaline medium & $\begin{array}{l}\eta_{10}=254 \mathrm{mV} \\
\text { Tafel slope: } 32 \mathrm{mV} \\
\text { decade }^{-1}\end{array}$ & $\begin{array}{l}\text { Metal and oxygen } \\
\text { vacancies }\end{array}$ & [216] \\
\hline $\mathrm{Ni}-\mathrm{Fe}-\mathrm{MOF}$ & Solvothermal & OER & Alkaline medium & $\begin{array}{l}\eta_{10}=221 \mathrm{mV} \\
\text { Tafel slope }=56 \mathrm{mV} \\
\text { decade }^{-1}\end{array}$ & $\begin{array}{l}\text { Fe constitutes the } \\
\text { active site }\end{array}$ & [307] \\
\hline CoFe LDH & $\begin{array}{l}\text { Hydrothermal and } \\
\text { Water-plasma-ena- } \\
\text { bled exfoliation }\end{array}$ & OER & Alkaline medium & $\begin{array}{l}\eta_{10}=232 \mathrm{mV} \\
\text { Tafel slope }=36 \mathrm{mV} \\
\text { decade }^{-1}\end{array}$ & $\begin{array}{l}\text { As-exfoliated increased } \\
\text { active sites and } \\
\text { multi-vacancies }\end{array}$ & [215] \\
\hline CoCo-LDH & $\begin{array}{l}\text { Soft template } \\
\text { method }\end{array}$ & OER & Alkaline medium & $\begin{array}{l}\eta_{10}=319 \mathrm{mV} \\
\text { Tafel slope }=42 \mathrm{mV} \\
\text { decade }^{-1}\end{array}$ & $\begin{array}{l}\text { More highly active } \\
\text { edge sites with lower } \\
\text { coordination number } \\
\text { and mass diffusion } \\
\text { promotion }\end{array}$ & [346] \\
\hline $\mathrm{Ni}(\mathrm{OH})_{2}$ & Chemical etching & OER & Alkaline medium & $\begin{array}{l}\eta_{10}=335 \mathrm{mV} \\
\text { Tafel slope }=65 \mathrm{mV} \\
\text { decade }^{-1}\end{array}$ & $\begin{array}{l}\text { Holes developed inside } \\
\text { the sheet structure } \\
\text { supply tremendous } \\
\text { permeable channels } \\
\text { for ions adsorption } \\
\text { and transportation }\end{array}$ & [344] \\
\hline BP & $\begin{array}{l}\text { Liquid phase exfolia- } \\
\text { tion }\end{array}$ & OER & Alkaline medium & $\begin{array}{l}\text { Onset }=1.45 \mathrm{~V}, \\
\eta_{10}=300 \mathrm{mV} \\
\text { Tafel slope }=88 \mathrm{mV} \\
\text { decade }^{-1}\end{array}$ & $\begin{array}{l}\text { Reduction in thickness } \\
\text { generates active sites } \\
\text { and improves specific } \\
\text { surface area }\end{array}$ & [283] \\
\hline Ni-MOF@Fe-MOF & Ultrasonication & OER & Alkaline medium & $\begin{array}{l}\eta_{10}=265 \mathrm{mV} \\
\text { Tafel slope }=82 \mathrm{mV} \\
\text { decade }^{-1}\end{array}$ & $\begin{array}{l}\text { Hybridization and } \\
\text { cooperativity } \\
\text { between } \mathrm{Ni} \text { and } \mathrm{Fe}\end{array}$ & [289] \\
\hline $\mathrm{Co}-\mathrm{C}_{3} \mathrm{~N}_{4} / \mathrm{CNT}$ & $\begin{array}{l}\text { Polycondensation } \\
\text { reactions, and acid } \\
\text { leaching process }\end{array}$ & ORR and OER & Alkaline medium & $\begin{aligned} \text { HER onset } & =0.9 \mathrm{~V}, \\
\text { OER onset } & =1.5 \mathrm{~V} \\
\text { Tafel slope } & =68.4 \mathrm{mV} \\
\text { decade }^{-1} & \end{aligned}$ & $\mathrm{M}-\mathrm{N}_{2}$ coordination & [171] \\
\hline $\mathrm{Fe}-\mathrm{Co} / \mathrm{N}-\mathrm{rGO}-\mathrm{Al}$ & Solvothermal & ORR & Alkaline medium & $\begin{array}{c}\text { Onset }=0.98 \mathrm{~V} \\
\text { half-wave poten- } \\
\text { tial }=0.84 \mathrm{~V}\end{array}$ & $\begin{array}{l}\text { Four electron transfer } \\
\text { mechanism and a } \\
\text { lower } \mathrm{HO}^{2-} \text { yield }\end{array}$ & [347] \\
\hline
\end{tabular}


Table 2 (continued)

\begin{tabular}{|c|c|c|c|c|c|c|}
\hline 2D nanomaterials & Synthesis & Application & Conditions & Performance or parameter & $\begin{array}{l}\text { Activity origin or } \\
\text { mechanism }\end{array}$ & Refs \\
\hline $\mathrm{Pd}$ & Thermal treatment & ORR & Alkaline medium & $\begin{array}{l}\text { Mass activity } \\
(0.85 \mathrm{~V})=21.1 \mathrm{~mA} \mathrm{mg}^{-1} \\
\text { electron transfer num- } \\
\text { ber }=3.73-3.85\end{array}$ & $\begin{array}{l}\text { Unique structural } \\
\text { features }\end{array}$ & [237] \\
\hline $\mathrm{WSe}_{2}$ & $\begin{array}{l}\text { Chemical vapor trans- } \\
\text { portation }\end{array}$ & $\mathrm{CO}_{2} \mathrm{RR}$ & Acidic media & $\begin{array}{l}\text { Current den- } \\
\text { sity }=18.95 \mathrm{~mA} \mathrm{~cm}^{-2} \\
\mathrm{CO} \text { formation turnover } \\
\text { frequency }=0.28 \mathrm{~s}^{-1} \\
\text { Overpotential }=54 \mathrm{mV}\end{array}$ & $\begin{array}{l}\text { Presence of ionic liq- } \\
\text { uids and high density } \\
\text { of edges }\end{array}$ & [349] \\
\hline $\mathrm{Ru} / \mathrm{MgAl}$ & Wet impregnation & $\mathrm{CO}_{2} \mathrm{RR}$ & $\begin{array}{l}\text { Gas phase } \\
\text { reaction }\end{array}$ & $\begin{array}{l}\mathrm{CO}_{2} \text { conversion }=85 \% \\
\mathrm{CH}_{4} \text { yield }=84 \%\end{array}$ & $\begin{array}{l}\text { Non-thermal plasma } \\
\text { (NTP) activated } \mathrm{CO}_{2} \\
\text { hydrogenation }\end{array}$ & [348] \\
\hline
\end{tabular}

photoexcitation degradation in $\mathrm{BiOCl}$ nanomaterials, and the direct semiconductor degradation efficiencies were 99\% and 59\% in $\mathrm{BiOCl}-001$ and $\mathrm{BiOCl}-010$, respectively. An indirect semiconductor exhibited a lower photocatalytic activity compared to direct semiconductor, and the $\mathrm{BiOCl}$ nanomaterials demonstrated higher photoexcitation performance under UV light than under visible light. In addition, Yang et al. constructed $\mathrm{BiOCl}$ and $\mathrm{BiOCl}-\mathrm{OH}$ photocatalysts for efficient photocatalysis and degradation of Rhodamine B dye in wastewater illuminated by UV light at a wavelength of $365 \mathrm{~nm}$, as illustrated in Fig. 6b, c [333]. The BiOCl-OH exhibited an enhanced photocatalysis compared to pure $\mathrm{BiOCl}$ since the UV light induced increased the number of oxygen vacancies, and the peaks in the FT-IR spectra correspond to hydroxyl groups and indicate the significant role of hydroxyl groups in the photocatalytic activity for Rhodamine B dye degradation
[333]. Zheng et al. developed a layered hetero-structured black phosphorous/graphitic carbon nitride (BP/CN) nanomaterial to obtain efficient photocatalysis for dye degradation and produce the highly reactive oxygen species of $\mathrm{H}_{2} \mathrm{O}_{2}$, as shown in Fig. 7 [334].

\subsection{Elimination of Toxicants}

Phenolic compounds are highly toxic organic pollutants that pollute water. These compounds are derived from pharmaceutical, printing, dyeing, pesticide and oil refining industries. The presence of phenols in industrial sewage makes surface water extremely vulnerable to pollution. The search for efficient and safe degradation technologies is therefore worthy of worldwide attention [37]. For example, Liu and co-workers found that a hetero-junctioned photocatalysts based on $\mathrm{g}-\mathrm{C}_{3} \mathrm{~N}_{4} / \mathrm{Bi}_{2} \mathrm{WO}_{6} / \mathrm{rGO}$ (incorporated with $3 \mathrm{wt} \%$ of
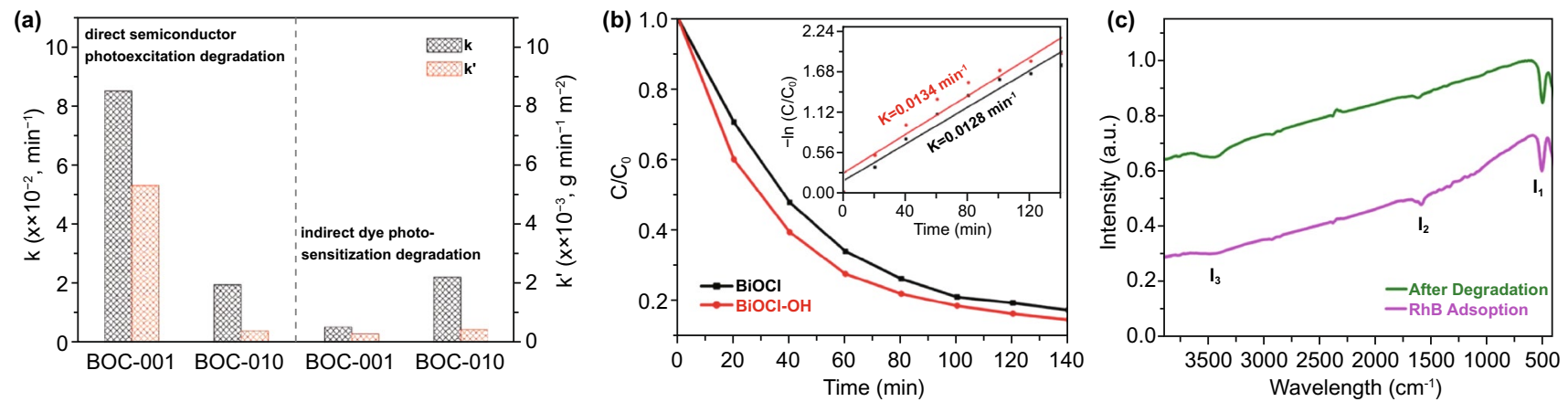

Fig. 6 Catalytic degradation applications of 2D nanomaterials. a Direct semiconductor photoexcitation pollutant degradation in single-crystalline BiOCl nanosheets under UV light. Reproduced with permission [332]. Copyright 2012, American Chemical Society. b, c BiOCl and BiOCl-OH photocatalyst for UV light driven photocatalytic dye degradation. Reproduced with permission [333]. Copyright 2017, American Chemical Society 

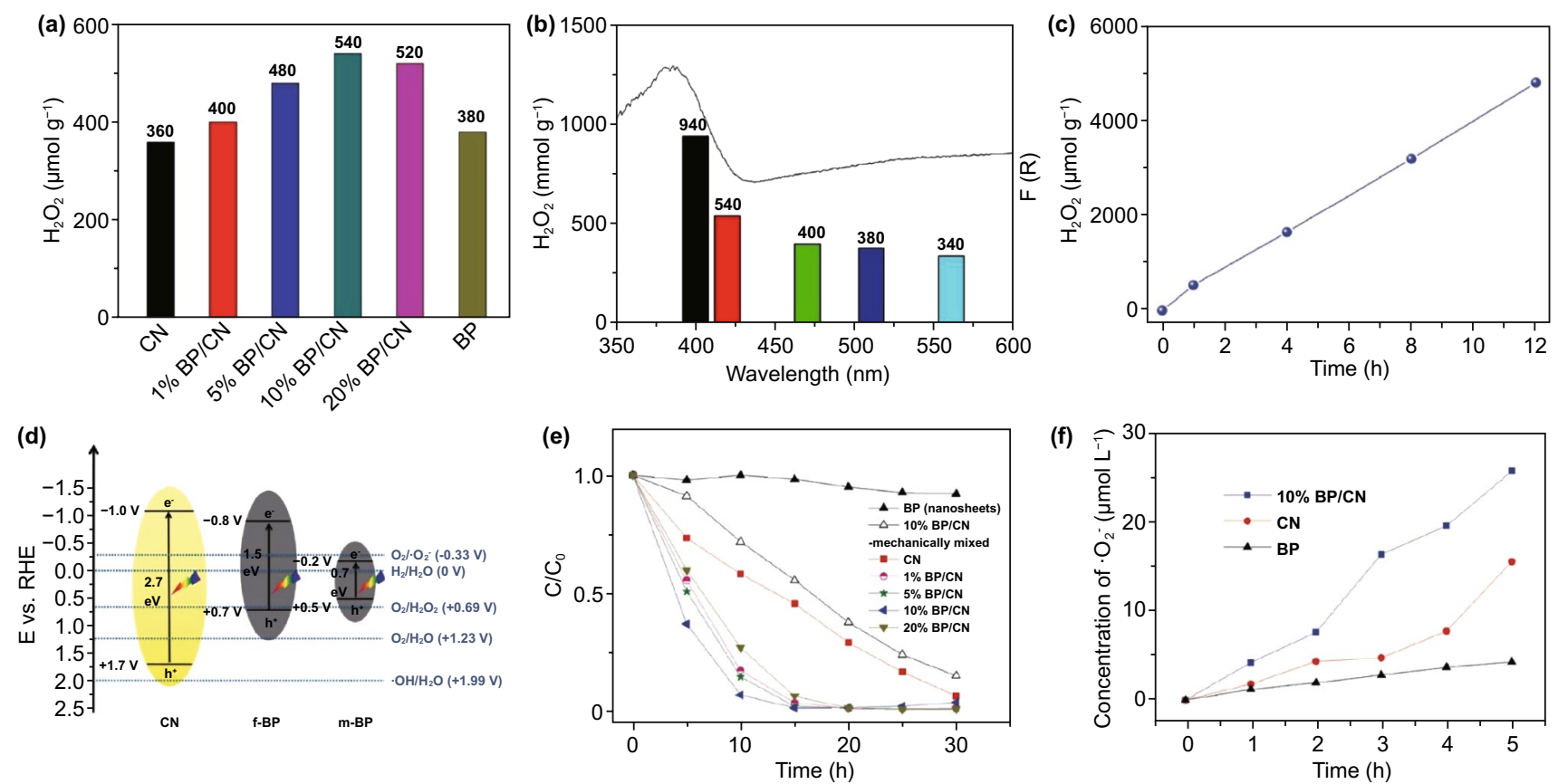

Fig. 7 Layered heterostructure structured black phosphorous/graphitic carbon nitride (BP/CN) nanomaterials for efficient photocatalysis. Reproduced with permission [334]. Copyright 2018, Wiley-VCH

rGO) exhibited a $86 \%$ and $98 \%$ reduction in ibuprofen by photocatalytic degradation under optical light and solar light irradiation, respectively [335].

Antibiotics are able to cause irreversible damage due to their easy accumulation in the human body, and photocatalytic oxidation has been applied as an efficient approach to antibiotics removal for wastewater treatment. Norvill and coworkers demonstrated that the antibiotic tetracycline could be reduced by $93 \%$ and $99 \%$ with a biomass concentration and chemical oxygen demand (COD) at hydraulic retention times of 4 and 7 days under summer-like conditions, although the lower photodegradation during the winter can lead to a reduced overall removal efficiency [336]. These results are the first to provide an effective demonstration of tetracycline removal in an outdoor wastewater environment and demonstrate that algal wastewater treatment provides a higher removal capacity compared to conventional biological wastewater treatment.

\subsection{Hydrogen Evolution Reaction (HER)}

Today, there is a need to develop new energy under the current worldwide circumstances of increasing environmental pollution and the energy crisis [337-339]. Hydrogen energy, as one of new and clean energy resources, possesses not only no secondary pollution, but also has a high energy density and has emerged as a low-carbon and zerocarbon energy. The HER is the cathodic reaction that can be described as $2 \mathrm{H}^{+}+2 \mathrm{e}^{-} \rightarrow \mathrm{H}_{2}$, known as the half part of water splitting [340]. For more detailed processes of the HER in acidic solutions, it can be divided into two main procedures including proton adsorption and hydrogen desorption, whose basic mechanisms are on basis of the Volmer mechanism, the Heyrovsky mechanism and the Tafel mechanism, where the adsorption sites of electrocatalysts play a significant role for the HER [12]. Numerous research on developing 2D material catalysts is exploring their attractive physicochemical properties as a potential catalyst for efficient HER activity with high efficiency [341]. For example, Ma and co-workers prepared ice-assisted exfoliated $\mathrm{BP} / \mathrm{g}-\mathrm{C}_{3} \mathrm{~N}_{4}$ nanosheets from bulk black phosphorous, which exhibited the properties of high product quality, a low density of anomalous structural defects and large lateral size [183]. Figure 8 illustrates the corresponding properties of the synthesized $\mathrm{BP} / \mathrm{g}-\mathrm{C}_{3} \mathrm{~N}_{4}$ nanosheets. The absorption spectra for individual BP, $\mathrm{g}-\mathrm{C}_{3} \mathrm{~N}_{4}$ and $\mathrm{BP} / \mathrm{g}$ $\mathrm{C}_{3} \mathrm{~N}_{4}$ nanosheets indicate that the absorption band of the $\mathrm{BP}$ nanosheets ranges broadly in the UV, visible and NIR 

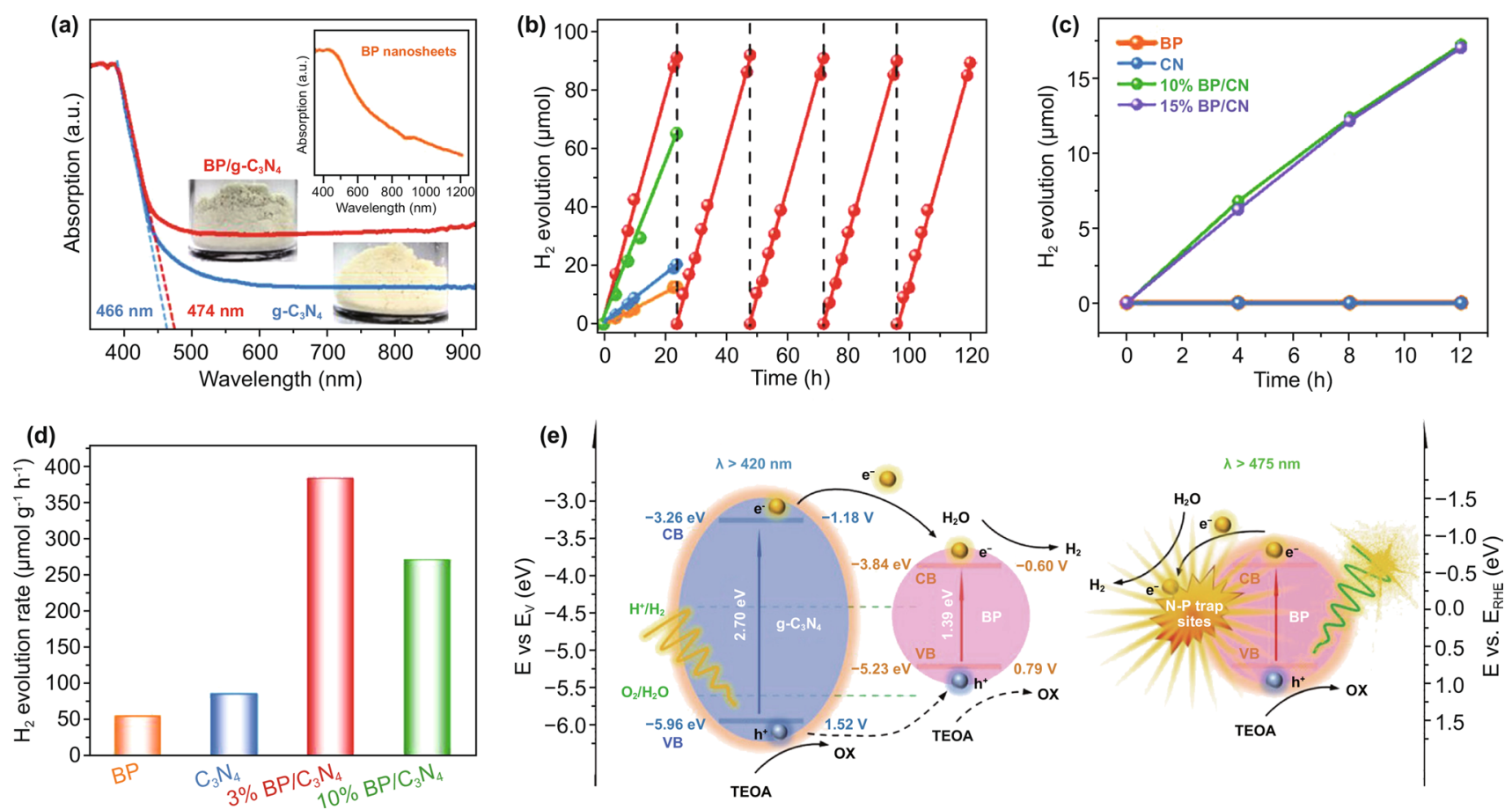

Fig. $8 \mathrm{BP} / \mathrm{g}-\mathrm{C}_{3} \mathrm{~N}_{4}$ nanosheets with properties of high-quality, large lateral size and lower anomalous structural defects for high efficiency catalytic $\mathrm{H}_{2}$ production. Reproduced with permission [183]. Copyright 2019, Wiley-VCH

regions, while the absorption edges of $\mathrm{g}_{-} \mathrm{C}_{3} \mathrm{~N}_{4}$ and $\mathrm{BP} / \mathrm{g}-$ $\mathrm{C}_{3} \mathrm{~N}_{4}$ nanosheets are $\sim 466$ and $\sim 474 \mathrm{~nm}$, respectively. In addition, the differing component ratios for $\mathrm{BP} / \mathrm{g}-\mathrm{C}_{3} \mathrm{~N}_{4}$ nanosheets have been analyzed, where the $\mathrm{BP} / \mathrm{g}-\mathrm{C}_{3} \mathrm{~N}_{4}$ nanosheets provide a clear photocatalytic enhancement for the HER compared to individual $\mathrm{BP}$ and $\mathrm{g}_{-} \mathrm{C}_{3} \mathrm{~N}_{4}$ catalysts, in terms of both photocatalytic hydrogen generation rate and total amount of product. The existence of $\mathrm{BP}$ enlarges the absorption band of the $\mathrm{BP} / \mathrm{g}-\mathrm{C}_{3} \mathrm{~N}_{4}$ and the addition of g- $\mathrm{C}_{3} \mathrm{~N}_{4}$ not only preserves the BP against oxidization, but it also provides a shallow interface of trapped charge sites for promotion of electric carrier separation in the composite photocatalysts, which reduces the limitations of fast carrier recombination in the $\mathrm{BP}$ or $\mathrm{g}-\mathrm{C}_{3} \mathrm{~N}_{4}$ nanosheets.

\subsection{Oxygen Evolution Reaction (OER)}

As the other half part of water splitting, the OER can be regarded as an oxidative reaction that demands four electrons and proton transfer, resulting in an overpotential requirement and a kinetically sluggish response [342-344]. On account of its atomic level thickness, large specific area and large amount of surface atoms, 2D LDH nanosheets are able to realize a significant improvement in catalytic performance [343]. Song et al. presented an orthogonal approach for catalytic OER enhancement using layered LDH nanosheets which were processed via liquid phase exfoliation [345]. As can be seen in Fig. 9, the bulk-layered LDHs exhibit a lower OER performance compared to exfoliated singlelayer LDH nanosheets. In addition, Qin et al. synthesized a 2D CoCo-LDH nanomesh as an OER electrocatalyst, where there were abundant high activity atoms with low ligancy, and the mesoporous structure of the CoCo-LDH nanomesh improved the diffusion of reactants and products, as illustrated in Fig. 10 [346]. The onset overpotential and the overpotential $\left(\eta_{10}\right)$ of the CoCo-LDH nanomesh were decreased to $220 \mathrm{mV}$ and $319 \mathrm{mV}$, respectively.

\subsection{Carbon Dioxide Reduction Reaction $\left(\mathrm{CO}_{2} \mathrm{RR}\right)$}

Currently, scientists have found that the $\mathrm{CO}_{2}$ concentration in the atmosphere surpasses the previous level of 23 million years and is increasing at an unprecedented rate [347]. $\mathrm{CO}_{2}$ is considered to be one of the most potent greenhouse 

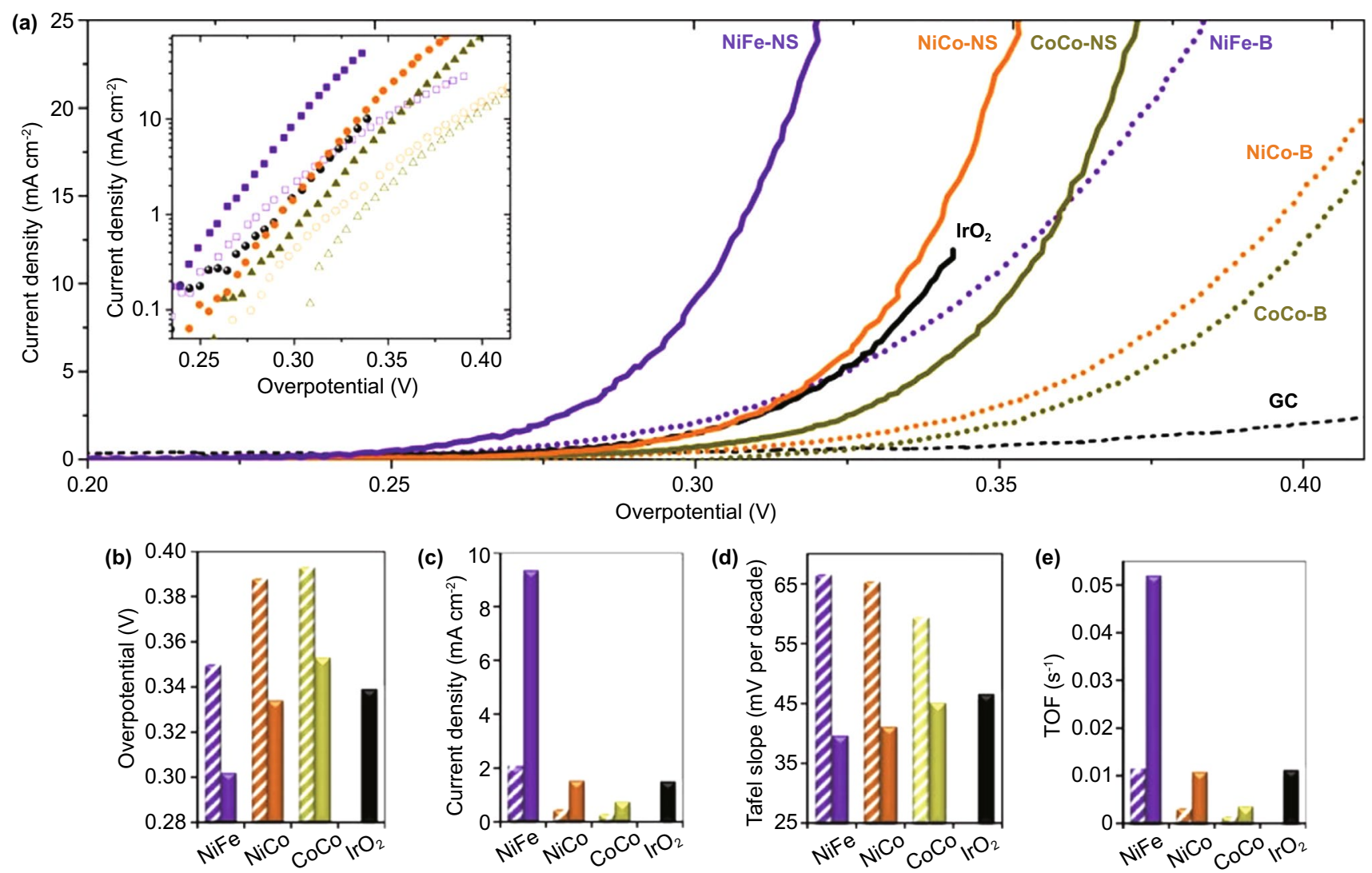

Fig. 9 Layered double hydroxide (LDH) nanosheets for catalytic oxygen evolution reaction (OER) enhancement. Reproduced with permission [345]. Copyright 2014, Springer Nature

pollutants, and the increase in $\mathrm{CO}_{2}$ concentration is closely related to climate change [348]. The capture and efficient use of $\mathrm{CO}_{2}$ is an urgent global problem [349]. As a result, 2D layered nanomaterials have attracted effort in terms of photocatalysis and electrocatalysis for $\mathrm{CO}_{2} \mathrm{RR}$ applications to transform $\mathrm{CO}_{2}$ into nontoxic organics [350]. Ye and coworkers realized an efficient $\mathrm{CO}_{2} \mathrm{RR}$ with a $\mathrm{CO}_{2}$ adsorption capacity of $103.8 \mathrm{~cm}^{3} \mathrm{~g}^{-1}$ for homogeneous $\mathrm{Zn}-\mathrm{MOF}$ nanomaterials with a $4.7 \mathrm{~nm}$ layer thickness, as shown in Fig. 11a, b [350]. In comparison with bulk MOFs of low efficiency, the synergistic effect of prolonged lifetime of photogenerated electric carriers offers the possibility of using $2 \mathrm{D}$ layered MOF nanosheets with desirable catalytic $\mathrm{CO}_{2} \mathrm{RR}$ activity. Zhao's group exploited $2 \mathrm{D} \mathrm{ZnO}$ for photocatalytic $\mathrm{CO}_{2} \mathrm{RR}$, as shown in Fig. 11c-e [351]. Compared with their bulk counterpart, the $2 \mathrm{D} \mathrm{ZnO}$ nanosheets have the advantages of desirable bandgap, optical absorbance and large surface catalytic active sites for $\mathrm{CO}_{2} \mathrm{RR}$.

\subsection{Cancer Therapy}

During recent decades, cancer is one of great threats to humankind and societal health, which inspires the development of functional nanomaterials with desirable characteristics and suitability for cancer therapy [352-361]. Du's group demonstrated cancer therapy by using $2 \mathrm{D}$ nanomaterials based on rare-earth metals [362, 363]. Dai et al. realized noble ablation of tumors that was attributed to the combination of physiochemical properties and photocatalytic effect in hydrothermally synthesized 2D O-BiOCl-PVP nanosheets, where the oxygen vacancies were constructed on material surfaces via UV light irradiation, as illustrated in Fig. 12 [364]. This recent work provides a new direction for defect engineering strategy of nanomaterials and enlarges the biomedical applications of 2D layered nanomaterials.

Here, various catalytic applications of 2D nanomaterials have been discussed in this section that contain 
(a)

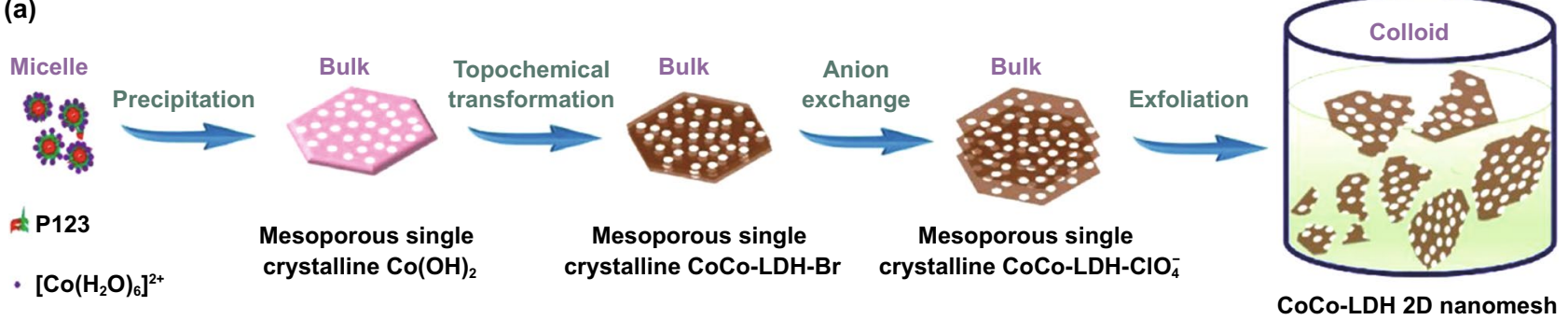

(b)

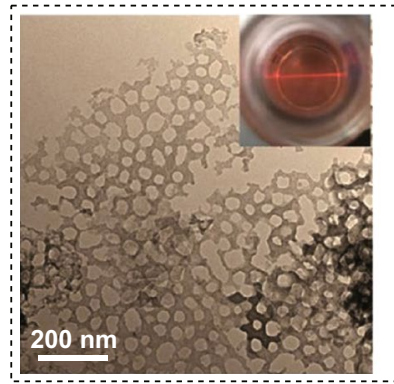

(d)
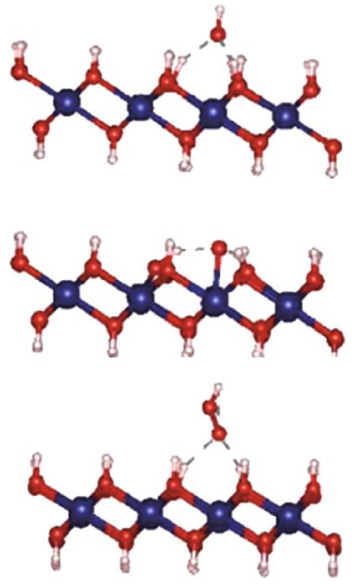
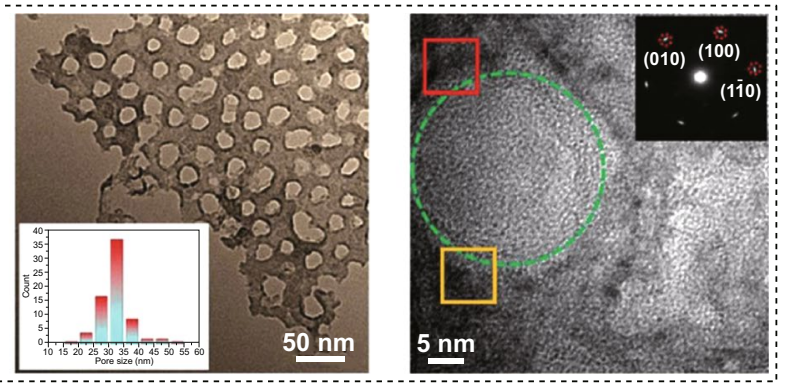

(c)

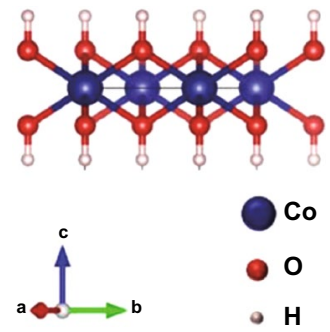

Fig. 10 CoCo-LDH 2D nanomesh for enhanced oxygen evolution. Reproduced with permission [346]. Copyright 2019, Wiley-VCH

environmental treatment and biochemical technologies including dye degradation, elimination of toxicant, HER, $\mathrm{OER}, \mathrm{CO}_{2} \mathrm{RR}$, as well as cancer therapy.

\section{Future Perspective and Challenges}

We now provide an overall perspective on recent progress in 2D nanomaterials for photocatalytic and electrocatalytic applications. This begins with the range of structures and synthesis approaches. We then focus on the emerging strategies for improving catalytic properties by enhancing light

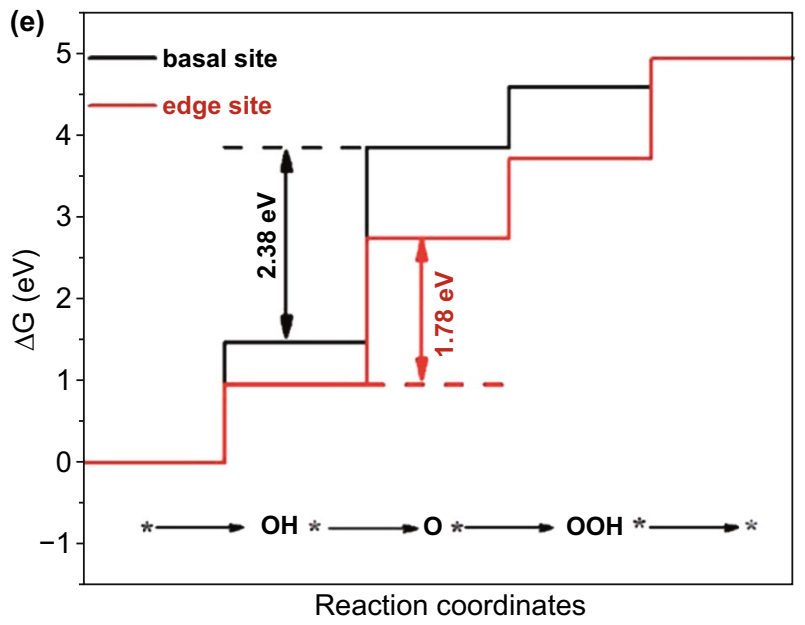

absorption ability, increasing reactive sites, accelerating carrier separation and charge migration, and improving surface reaction. Their applications in the realm of environmental treatment and biochemical technologies are highlighted.

Compared with traditional bulk nanoscale catalysts, 2D layered nanomaterials in the fields of photocatalysis and electrocatalysis exhibit specific advantages such as an exposure to more active sites, being more conducive to reactant diffusion and a larger specific surface area. In addition, much effort in optimizing these materials have been devoted to enhancement of stability, electrical and mechanical properties through changing layer thickness, surface modification 

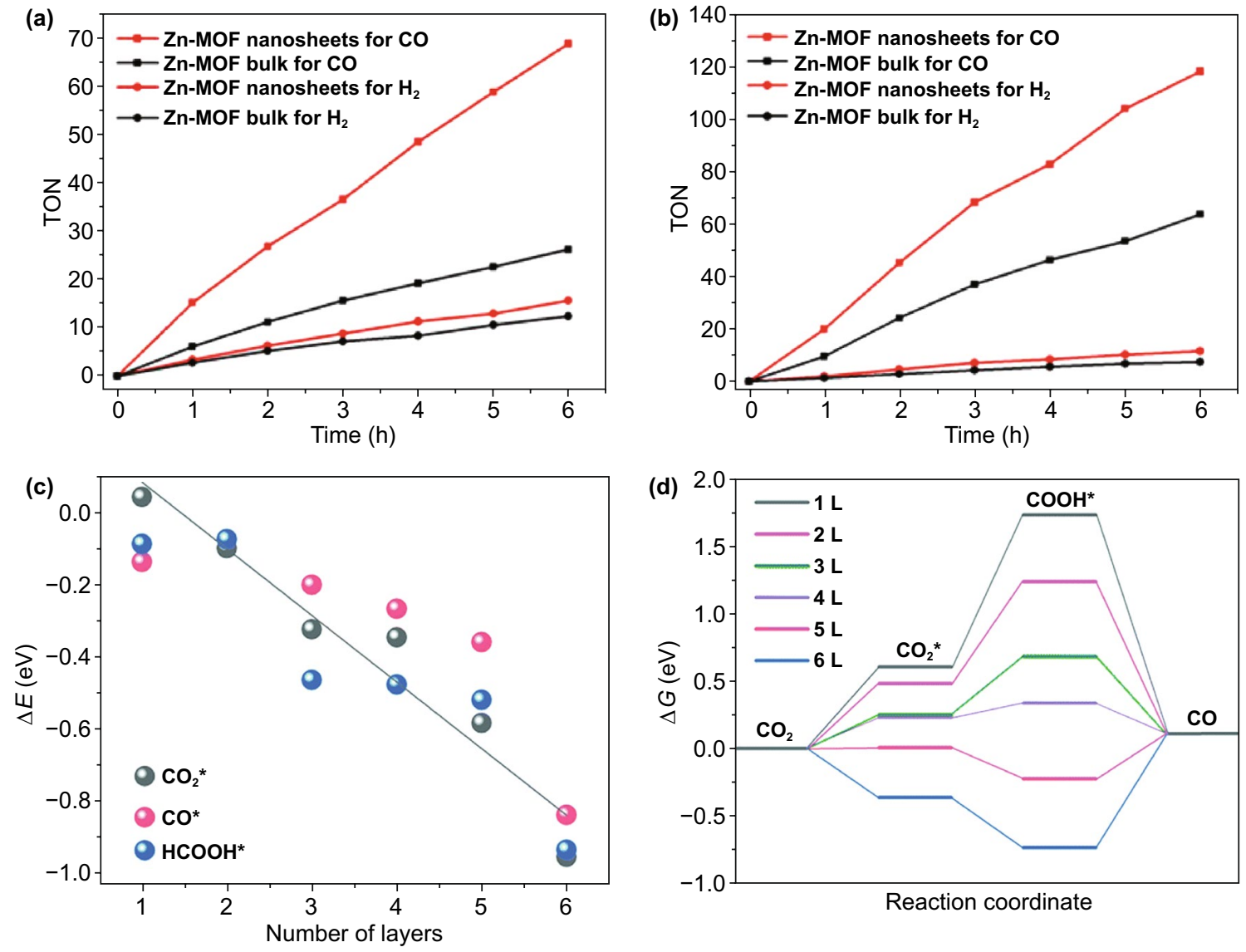

(e)
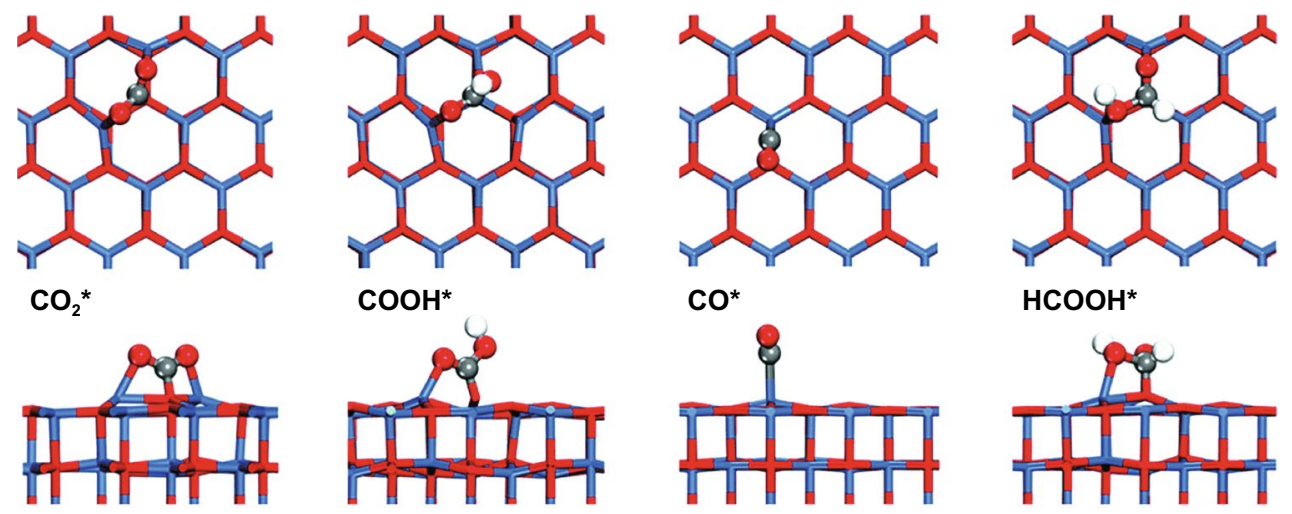

Fig. 11 2D nanomaterials for efficient photocatalytic $\mathrm{CO}_{2}$ conversion systems. a, b Ultrathin 2D Zn-MOF nanosheets for photoreduction of $\mathrm{CO}_{2}$ to CO. Reproduced with permission [350]. Copyright 2018, Elsevier. c-e 2D ZnO nanomaterials for selective photoreduction of $\mathrm{CO}_{2}$. Reproduced with permission [351]. Copyright 2019, Royal Society of Chemistry

and external stimulation. Research progress and future directions in 2D nanocatalysts are aiming to tackle a range of issues and challenges, which are summarized below:

(1) The macroscopic and controlled production of 2D nanomaterials is key to the practical application of catalysis. Chemical vapor deposition and liquid phase dissection are potential synthesis approaches for the production of 2D layered nanomaterials. Recently, in situ characterization equipment has been developed that is able to detect thermodynamic and kinetic reactions during material synthesis, which can be highly beneficial for developing a detailed understanding of the growth mechanisms of 2D nanomaterials. In addi- 
(a)
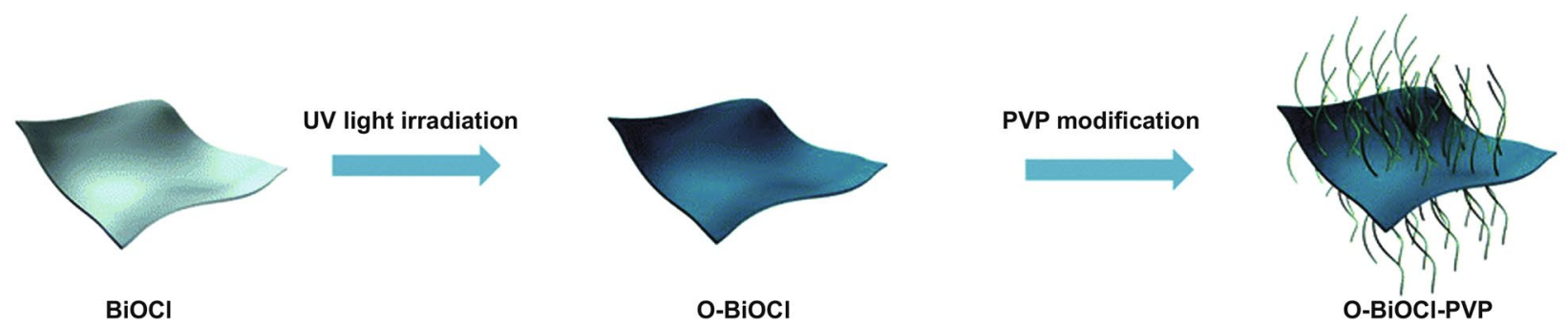

(b)
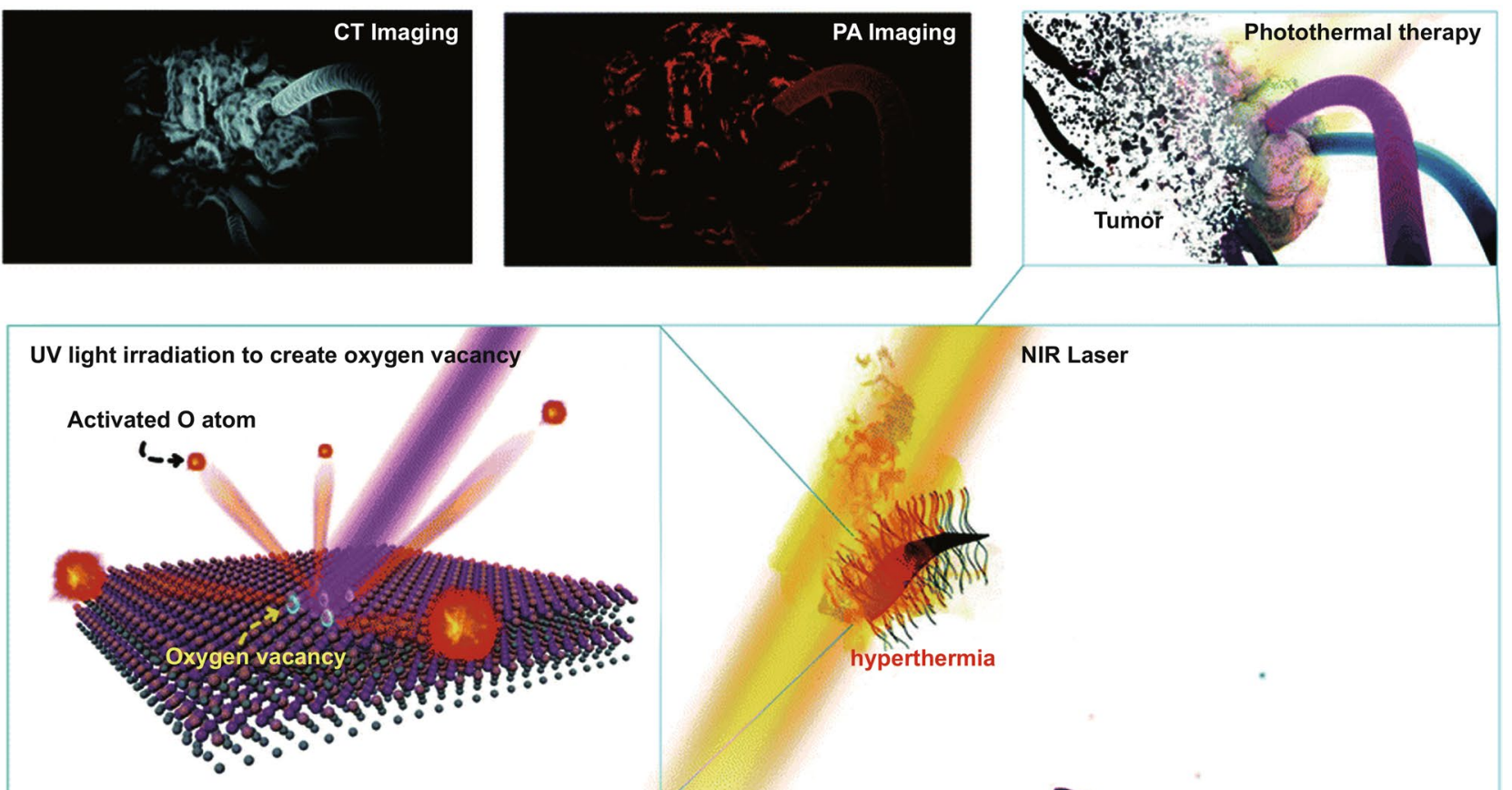

NIR Laser

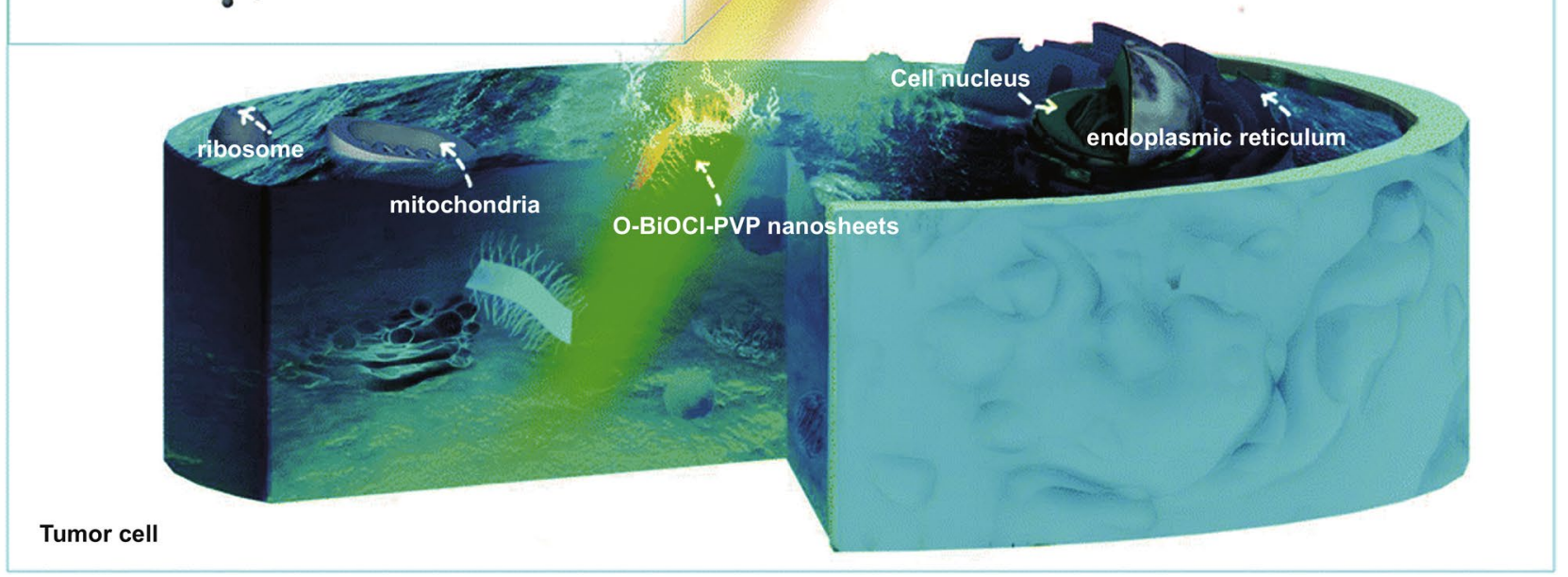

Fig. 12 2D BiOCl nanosheets for photonic tumor ablation with the combination of physiochemical properties and photocatalytic effect. Reproduced with permission [364]. Copyright 2020, Royal Society of Chemistry

tion to existing progress in the manufacture of $2 \mathrm{D}$ nanocatalysts within a controlled microenvironment, the use of confined synthesis could be a significant growth approach that is able to operate at a molecule level to offer precise control for the synthesis for $2 \mathrm{D}$ nanomaterials for catalytic applications. In the longer term, the large-scale preparation of controllable structures of non-layered atomic thickness nanosheets with intrinsic catalytic activity remains to be developed, which faces significant challenges, especially in achiev- 
ing precise control for the production of high-quality and homogenous 2D nanomaterials.

(2) In general, catalytic activity is closely related to the structure and surface characteristics of a 2D nanocatalyst, which can adjust the electronic properties and electron transfer. The structure and surface characteristics of 2D nanomaterials is related to its dimensions (interlayer spacing, thickness and transverse dimensions), exposed surface atom density, the existence of surface impurities, additional functional groups and surface energy states. It is worth highlighting that the abundant edges of 2D nanomaterials lead to a high electron transfer activity, compared to basal planes. Thus, the design of 2D catalytic nanomaterials can benefit from the regulation of defects, heteroatom-doping and the tuning of edges and planes.

(3) The creation of 2D nanomaterials with hybrid composite structures has become topic of intense research interest, where two or more compounds with a different degree of anisotropy and characteristics create new possibilities in the design of 2D nanocomposites with multi-functional and tailored properties for catalysis applications. In addition, heterostructures that are built by combining individual materials are showing promising potential in providing control of structure and electronic properties. Future directions for catalytic-related applications in 2D nanomaterials could therefore involve the creation of sandwich structures, confined space structures, and strong electron interactions. In addition, the concept of coupling multiple systems provides new opportunities to enable multiple mechanisms to operative cooperatively. For example, piezoelectric semiconductors possess piezoelectricity and photovoltaic effects simultaneously. By combining these two effects, the photocatalytic activity can be enhanced by an internal piezoelectric field that couples both piezoelectric and photovoltaic effects.

(4) With regard to developing a greater theoretical understanding of the mechanisms of catalysis in 2D nanomaterials, there have been anastomotic models that link experimental results and theoretical analysis. However, current catalytic studies of 2D nanomaterials have placed an emphasis on catalytic activity enhancement, rather than the underlying science of the catalytic mechanisms, and much of research on 2D nanocatalystbased catalysis involves trial and error. In addition, the use of 2D layered nanocatalysts makes the reaction system more complex compared to 3D bulk materials; thus, new developments in the underlying science of 2D catalytic nanomaterials are beneficial for mate- rial design and discovery. For example, with regard to photocatalysis, the bandgap of a semiconductor for catalysts using light excitation is a dominant factor. The fundamental mechanisms and specific impact of the dimensionality of 2D nanomaterials and 3D bulk materials to control the energy bandgap positions remain unclear. To date, the theoretical catalytic mechanisms of 2D nanomaterials are still not easily applicable to real complex reaction systems, thus more theoretical and fundamental studies on catalytic mechanisms are worthy of study.

(5) In addition to technology development for mass production, the ability for catalyst shaping is of interest for end-use applications. When 2D nanomaterials are used in the form of loose powders they can agglomerate which can restrict their application. The potential of fixing 2D nanomaterials to a substrate provides a promising approach for improving their ease of use. For example, 2D nanomaterials could be epitaxially grown on the surface of other materials, be assembled into a foam, or be supported on carbon fiber paper or nickel foam for catalytic activity enhancement.

2D nanomaterials have become one of the most promising forms of catalysts applied to both photocatalysis and electrocatalysis, but it is merely on the threshold for comprehensive analysis both in terms of experimental data and theoretical understanding. By furthering our understanding in 2D nanocatalysts in terms of production, material design, hybridization, catalytic mechanisms, and applications, the potential of 2D nanomaterials for practical catalysis in the future will be more clearly understood. The intention of the review is therefore to inspire new efforts to accelerate the development of 2D layered materials for catalysis-related applications.

Acknowledgements This work was supported by the National Key R\&D Project from Minister of Science and Technology in China (No. 2016YFA0202701, No. 2018YFB2200500), the National Natural Science Foundation of China (No. 52072041, No. 61604012, No. 61974170), and the University of Chinese Academy of Sciences (Grant No. Y8540XX2D2).

Open Access This article is licensed under a Creative Commons Attribution 4.0 International License, which permits use, sharing, adaptation, distribution and reproduction in any medium or format, as long as you give appropriate credit to the original author(s) and the source, provide a link to the Creative Commons licence, and indicate if changes were made. The images or other third party material in this article are included in the article's Creative Commons licence, unless indicated otherwise in a credit line to the 
material. If material is not included in the article's Creative Commons licence and your intended use is not permitted by statutory regulation or exceeds the permitted use, you will need to obtain permission directly from the copyright holder. To view a copy of this licence, visit http://creativecommons.org/licenses/by/4.0/.

\section{References}

1. Q. Wang, K. Domen, Particulate photocatalysts for lightdriven water splitting: Mechanisms, challenges, and design strategies. Chem. Rev. 120, 919-985 (2020). https://doi.org/ 10.1021/acs.chemrev.9b00201

2. S.S. Chen, T. Takata, K. Domen, Particulate photocatalysts for overall water splitting. Nat. Rev. Mater. 2, 17050 (2017). https://doi.org/10.1038/natrevmats.2017.50

3. S.C. Sun, G.Q. Shen, J.W. Jiang, W.B. Mi, X.L. Liu et al., Boosting oxygen evolution kinetics by Mn-N-C motifs with tunable spin state for highly efficient solar-driven water splitting. Adv. Energy Mater. 9, 1901505 (2019). https://doi.org/ 10.1002/aenm.201901505

4. L. Pan, M.H. Ai, C.Y. Huang, L. Yin, X. Liu et al., Manipulating spin polarization of titanium dioxide for efficient photocatalysis. Nat. Commun. 11, 418 (2020). https://doi.org/10. 1038/s41467-020-14333-w

5. X.D. Wang, J.H. Song, J. Liu, Z.L. Wang, Direct-current nanogenerator driven by ultrasonic waves. Science 316, 102-105 (2007). https://doi.org/10.1126/science.1139366

6. A. Fujishima, K. Honda, Electrochemical photolysis of water at a semiconductor electrode. Nature 238, 37-38 (1972). https://doi.org/10.1038/238037a0

7. X.B. Chen, S.H. Shen, L.J. Guo, S.S. Mao, Semiconductorbased photocatalytic hydrogen generation. Chem. Rev. 110, 6503-6570 (2010). https://doi.org/10.1021/cr1001645

8. M.D. Karkas, O. Verho, E.V. Johnston, B. Akermark, Artificial photosynthesis: Molecular systems for catalytic water oxidation. Chem. Rev. 114, 11863-12001 (2014). https://doi. org/10.1021/cr400572f

9. H.L. Wang, L.S. Zhang, Z.G. Chen, J.Q. Hu, S.J. Li et al., Semiconductor heterojunction photocatalysts: Design, construction, and photocatalytic performances. Chem. Soc. Rev. 43, 5234-5244 (2014). https://doi.org/10.1039/c4cs00126e

10. H. Jin, C. Guo, X. Liu, J. Liu, A. Vasileff et al., Emerging two-dimensional nanomaterials for electrocatalysis. Chem. Rev. 118, 6337-6408 (2018). https://doi.org/10.1021/acs. chemrev.7b00689

11. M.K. Debe, Electrocatalyst approaches and challenges for automotive fuel cells. Nature 486, 43-51 (2012). https://doi. org/10.1038/nature11115

12. X. Chia, M. Pumera, Characteristics and performance of two-dimensional materials for electrocatalysis. Nat. Catal. 1, 909-921 (2018). https://doi.org/10.1038/s41929-018-0181-7

13. Y. Jiao, Y. Zheng, M.T. Jaroniec, S.Z. Qiao, Design of electrocatalysts for oxygen- and hydrogen-involving energy conversion reactions. Chem. Soc. Rev. 44, 2060-2086 (2015). https://doi.org/10.1039/C4CS00470A

14. E. Roduner, Selected fundamentals of catalysis and electrocatalysis in energy conversion reactions-a tutorial. Catal. Today 309, 263-268 (2018). https://doi.org/10.1016/j.cattod.2017. 05.091

15. S. Linic, U. Aslam, C. Boerigter, M. Morabito, Photochemical transformations on plasmonic metal nanoparticles. Nat. Mater. 14, 567-576 (2015). https://doi.org/10.1038/nmat4281

16. H. Tada, T. Kiyonaga, S. Naya, Rational design and applications of highly efficient reaction systems photocatalyzed by noble metal nanoparticle-loaded titanium(IV) dioxide. Chem. Soc. Rev. 38, 1849-1858 (2009). https://doi.org/10. $1039 / \mathrm{b} 822385 \mathrm{~h}$

17. S. Linic, P. Christopher, D.B. Ingram, Plasmonic-metal nanostructures for efficient conversion of solar to chemical energy. Nat. Mater. 10, 911-921 (2011). https://doi.org/10. 1038/Nmat3151

18. N. Tian, Z.Y. Zhou, S.G. Sun, Y. Ding, Z.L. Wang, Synthesis of tetrahexahedral platinum nanocrystals with high-index facets and high electro-oxidation activity. Science 316, 732-735 (2007). https://doi.org/10.1126/science.1140484

19. B.H. Wu, N.F. Zheng, Surface and interface control of noble metal nanocrystals for catalytic and electrocatalytic applications. Nano Today 8, 168-197 (2013). https://doi.org/10. 1016/j.nantod.2013.02.006

20. S. Koh, P. Strasser, Electrocatalysis on bimetallic surfaces: Modifying catalytic reactivity for oxygen reduction by voltammetric surface dealloying. J. Am. Chem. Soc. 129, 12624-12625 (2007). https://doi.org/10.1021/ja0742784

21. B.H. Wu, Y.J. Kuang, X.H. Zhang, J.H. Chen, Noble metal nanoparticles/carbon nanotubes nanohybrids: Synthesis and applications. Nano Today 6, 75-90 (2011). https://doi.org/10. 1016/j.nantod.2010.12.008

22. X.F. Yang, A.Q. Wang, B.T. Qiao, J. Li, J.Y. Liu et al., Single-atom catalysts: A new frontier in heterogeneous catalysis. Acc. Chem. Res. 46, 1740-1748 (2013). https://doi.org/10. 1021/ar300361m

23. X.Y. Chia, A.Y.S. Eng, A. Ambrosi, S.M. Tan, M. Pumera, Electrochemistry of nanostructured layered transition-metal dichalcogenides. Chem. Rev. 115, 11941-11966 (2015). https://doi.org/10.1021/acs.chemrev.5b00287

24. M. Chhowalla, H.S. Shin, G. Eda, L.J. Li, K.P. Loh et al., The chemistry of two-dimensional layered transition metal dichalcogenide nanosheets. Nat. Chem. 5, 263-275 (2013). https://doi.org/10.1038/nchem.1589

25. D. Jariwala, T.J. Marks, M.C. Hersam, Mixed-dimensional van der Waals heterostructures. Nat. Mater. 16, 170-181 (2017). https://doi.org/10.1038/Nmat4703

26. S.Z. Butler, S.M. Hollen, L.Y. Cao, Y. Cui, J.A. Gupta et al., Progress, challenges, and opportunities in two-dimensional materials beyond graphene. ACS Nano 7, 2898-2926 (2013). https://doi.org/10.1021/nn400280c

27. G.R. Bhimanapati, Z. Lin, V. Meunier, Y. Jung, J. Cha et al., Recent advances in two-dimensional materials beyond 
graphene. ACS Nano 9, 11509-11539 (2015). https://doi. org/10.1021/acsnano.5b05556

28. M.M. Wu, T. Wu, M.Z. Sun, L. Lu, N. Li et al., General synthesis of large-area flexible bi-atomic subnano thin lanthanide oxide nanoscrolls. Nano Energy 78, 105318 (2020). https://doi.org/10.1016/j.nanoen.2020.105318

29. J. Xu, X.Y. Chen, Y.S. Xu, Y.P. Du, C.H. Yan, Ultrathin 2D rare-earth nanomaterials: Compositions, syntheses, and applications. Adv. Mater. 32, 1806461 (2020). https://doi. org/10.1002/adma.201806461

30. A.K. Geim, K.S. Novoselov, The rise of graphene. Nat. Mater. 6, 183-191 (2007). https://doi.org/10.1038/nmat1 849

31. H. Zhang, Ultrathin two-dimensional nanomaterials. ACS Nano 9, 9451-9469 (2015). https://doi.org/10.1021/acsna no. 5 b05040

32. M.S. Xu, T. Liang, M.M. Shi, H.Z. Chen, Graphene-like two-dimensional materials. Chem. Rev. 113, 3766-3798 (2013). https://doi.org/10.1021/cr300263a

33. J.Y. Tang, X.Y. Kong, B.J. Ng, Y.H. Chew, A.R. Mohamed et al., Midgap-state-mediated two-step photoexcitation in nitrogen defect-modified g- $\mathrm{C}_{3} \mathrm{~N}_{4}$ atomic layers for superior photocatalytic $\mathrm{CO}_{2}$ reduction. Catal. Sci. Technol. 9, 2335-2343 (2019). https://doi.org/10.1039/c9cy00449a

34. B. Luo, G. Liu, L. Wang, Recent advances in 2D materials for photocatalysis. Nanoscale 8, 6904-6920 (2016). https:// doi.org/10.1039/c6nr00546b

35. J. Kibsgaard, Z. Chen, B.N. Reinecke, T.F. Jaramillo, Engineering the surface structure of $\mathrm{MoS}_{2}$ to preferentially expose active edge sites for electrocatalysis. Nat. Mater. 11, 963-969 (2012). https://doi.org/10.1038/nmat3439

36. G. Wang, J. Tao, Y. Zhang, S. Wang, X. Yan et al., Engineering two-dimensional mass-transport channels of the $\mathrm{MoS}_{2}$ nanocatalyst toward improved hydrogen evolution performance. ACS Appl. Mater. Interfaces 10, 2540925414 (2018). https://doi.org/10.1021/acsami.8b07163

37. S. Zhang, B. Li, X. Wang, G. Zhao, B. Hu et al., Recent developments of two-dimensional graphene-based composites in visible-light photocatalysis for eliminating persistent organic pollutants from wastewater. Chem. Eng. J. 390, 124642 (2020). https://doi.org/10.1016/j.cej.2020.124642

38. H.L. You, Y.M. Jia, Z. Wu, X.L. Xu, W.Q. Qian et al., Strong piezo-electrochemical effect of multiferroic $\mathrm{BiFeO}_{3}$ square micro-sheets for mechanocatalysis. Electrochem. Commun. 79, 55-58 (2017). https://doi.org/10.1016/j.elecom.2017.04.017

39. W.Q. Qian, W.Y. Yang, Y. Zhang, C.R. Bowen, Y. Yang, Piezoelectric materials for controlling electro-chemical processes. Nano-Micro Lett. 12, 149 (2020). https://doi. org/10.1007/s40820-020-00489-Z

40. J. Wu, Z. Wu, W.Q. Qian, Y.M. Jia, Y. Wang et al., Electricfield-treatment-induced enhancement of photoluminescence in Er3+-doped $\left(\mathrm{Ba}_{0.95} \mathrm{Sr}_{0.05}\right)\left(\mathrm{Zr}_{0.1} \mathrm{Ti}_{0.9}\right) \mathrm{O} 3$ piezoelectric ceramic. Mater. Lett. 184, 131-133 (2016). https://doi.org/ 10.1016/j.matlet.2016.07.061
41. H. Wang, X. Liu, P. Niu, S. Wang, J. Shi et al., Porous twodimensional materials for photocatalytic and electrocatalytic applications. Matter 2, 1377-1413 (2020). https://doi.org/10. 1016/j.matt.2020.04.002

42. D. Qin, Y. Zhou, W. Wang, C. Zhang, G. Zeng et al., Recent advances in two-dimensional nanomaterials for photocatalytic reduction of $\mathrm{CO}_{2}$ : Insights into performance, theories and perspective. J. Mater. Chem. A 8, 19156-19195 (2020). https://doi.org/10.1039/d0ta07460h

43. K. Khan, A.K. Tareen, M. Aslam, R.U.R. Sagar, B. Zhang et al., Recent progress, challenges, and prospects in twodimensional photo-catalyst materials and environmental remediation. Nano-Micro Lett. 12, 167 (2020). https://doi. org/10.1007/s40820-020-00504-3

44. C. Tan, X. Cao, X.J. Wu, Q. He, J. Yang et al., Recent advances in ultrathin two-dimensional nanomaterials. Chem. Rev. 117, 6225-6331 (2017). https://doi.org/10.1021/acs. chemrev.6b00558

45. L. Pan, S. Sun, Y. Chen, P. Wang, J. Wang et al., Advances in piezo-phototronic effect enhanced photocatalysis and photoelectrocatalysis. Adv. Energy Mater. 10, 2000214 (2020). https://doi.org/10.1002/aenm.202000214

46. W. Qian, K. Zhao, D. Zhang, C.R. Bowen, Y. Wang et al., Piezoelectric material-polymer composite porous foam for efficient dye degradation via the piezo-catalytic effect. ACS Appl. Mater. Interfaces 11, 27862-27869 (2019). https://doi. org/10.1021/acsami.9b07857

47. S. Xu, W. Qian, D. Zhang, X. Zhao, X. Zhang et al., A coupled photo-piezo-catalytic effect in a bst-pdms porous foam for enhanced dye wastewater degradation. Nano Energy 77, 105305 (2020). https://doi.org/10.1016/j.nanoen.2020. 105305

48. X.N. Zang, W.S. Chen, X.L. Zou, J.N. Hohman, L.J. Yang et al., Self-assembly of large-area 2D polycrystalline transition metal carbides for hydrogen electrocatalysis. Adv. Mater. 30, 1805188 (2018). https://doi.org/10.1002/adma.20180 5188

49. M. Zhou, Z.L. Zhang, K.K. Huang, Z. Shi, R.G. Xie et al., Colloidal preparation and electrocatalytic hydrogen production of $\mathrm{MoS}_{2}$ and $\mathrm{WS}_{2}$ nanosheets with controllable lateral sizes and layer numbers. Nanoscale 8, 15262-15272 (2016). https://doi.org/10.1039/c6nr04775k

50. T.Y. Wang, L. Liu, Z.W. Zhu, P. Papakonstantinou, J.B. Hu et al., Enhanced electrocatalytic activity for hydrogen evolution reaction from self-assembled monodispersed molybdenum sulfide nanoparticles on an au electrode. Energy Environ. Sci. 6, 625-633 (2013). https://doi.org/10.1039/c2ee2 $3513 \mathrm{~g}$

51. G. Fiori, F. Bonaccorso, G. Iannaccone, T. Palacios, D. Neumaier et al., Electronics based on two-dimensional materials. Nat. Nanotechnol. 9, 768-779 (2014). https://doi.org/10. 1038/Nnano.2014.207

52. Y.F. Hao, M.S. Bharathi, L. Wang, Y.Y. Liu, H. Chen et al., The role of surface oxygen in the growth of large singlecrystal graphene on copper. Science 342, 720-723 (2013). https://doi.org/10.1126/science.1243879 
53. Y.Q. Wu, P.D. Ye, M.A. Capano, Y. Xuan, Y. Sui et al., Topgated graphene field-effect-transistors formed by decomposition of sic. Appl. Phys. Lett. 92, 092102 (2008). https://doi. org/10.1063/1.2889959

54. F. Torrisi, T. Hasan, W.P. Wu, Z.P. Sun, A. Lombardo et al., Inkjet-printed graphene electronics. ACS Nano 6, 2992-3006 (2012). https://doi.org/10.1021/nn2044609

55. B. Fallahazad, S. Kim, L. Colombo, E. Tutuc, Dielectric thickness dependence of carrier mobility in graphene with $\mathrm{HfO}_{2}$ top dielectric. Appl. Phys. Lett. 97, 123105 (2010). https://doi.org/10.1063/1.3492843

56. G. Nandamuri, S. Roumimov, R. Solanki, Remote plasma assisted growth of graphene films. Appl. Phys. Lett. 96, 154101 (2010). https://doi.org/10.1063/1.3387812

57. J. Zheng, H. Zhang, S.H. Dong, Y.P. Liu, C.T. Nai et al., High yield exfoliation of two-dimensional chalcogenides using sodium naphthalenide. Nat. Commun. 5, 2995 (2014). https://doi.org/10.1038/ncomms3995

58. B. Radisavljevic, A. Kis, Mobility engineering and a metalinsulator transition in monolayer $\mathrm{MoS}_{2}$. Nat. Mater. 12, 815820 (2013). https://doi.org/10.1038/Nmat3687

59. B. Radisavljevic, A. Kis, Measurement of mobility in dualgated $\mathrm{MoS}_{2}$ transistors. Nat. Nanotechnol. 8, 146-147 (2013). https://doi.org/10.1038/nnano.2013.30

60. H. Wang, L.L. Yu, Y.H. Lee, Y.M. Shi, A. Hsu et al., Integrated circuits based on bilayer $\mathrm{MoS}_{2}$ transistors. Nano Lett. 12, 4674-4680 (2012). https://doi.org/10.1021/nl302015v

61. G. Fiori, B.N. Szafranek, G. Iannaccone, D. Neumaier, Velocity saturation in few-layer $\mathrm{MoS}_{2}$ transistor. Appl. Phys. Lett. 103, 233509 (2013). https://doi.org/10.1063/1.4840175

62. Y.F. Yu, S.Y. Huang, Y.P. Li, S.N. Steinmann, W.T. Yang et al., Layer-dependent electrocatalysis of $\mathrm{MoS}_{2}$ for hydrogen evolution. Nano Lett. 14, 553-558 (2014). https://doi.org/10. 1021/nl403620g

63. M. Javaid, D.W. Drumm, S.P. Russo, A.D. Greentree, A study of size-dependent properties of $\mathrm{MoS}_{2}$ monolayer nanoflakes using density-functional theory. Sci. Rep. 7, 9775 (2017). https://doi.org/10.1038/s41598-017-09305-y

64. Y.L. Li, P.P. Li, J.S. Wang, Y.L. Yang, W.Q. Yao et al., Water soluble graphitic carbon nitride with tunable fluorescence for boosting broad-response photocatalysis. Appl. Catal. B-Environ. 225, 519-529 (2018). https://doi.org/10.1016/j.apcatb. 2017.12.017

65. X.W. Li, Y.J. Sun, T. Xiong, G.M. Jiang, Y.X. Zhang et al., Activation of amorphous bismuth oxide via plasmonic bi metal for efficient visible-light photocatalysis. J. Catal. 352, 102-112 (2017). https://doi.org/10.1016/j.jcat.2017.04.025

66. R.A. He, D.F. Xu, B. Cheng, J.G. Yu, W.K. Ho, Review on nanoscale bi-based photocatalysts. Nanoscale Horiz. 3, 464504 (2018). https://doi.org/10.1039/c8nh00062j

67. X.C. Du, J.W. Huang, J.J. Zhang, Y.C. Yan, C.Y. Wu et al., Modulating electronic structures of inorganic nanomaterials for efficient electrocatalytic water splitting. Angew. Chem. Int. Ed. 58, 4484-4502 (2019). https://doi.org/10.1002/anie. 201810104
68. Y.Q. Guo, K. Xu, C.Z. Wu, J.Y. Zhao, Y. Xie, Surface chemical-modification for engineering the intrinsic physical properties of inorganic two-dimensional nanomaterials. Chem. Soc. Rev. 44, 637-646 (2015). https://doi.org/10.1039/c4cs00302k

69. X. Long, G.X. Li, Z.L. Wang, H.Y. Zhu, T. Zhang et al., Metallic iron-nickel sulfide ultrathin nanosheets as a highly active electrocatalyst for hydrogen evolution reaction in acidic media. J. Am. Chem. Soc. 137, 11900-11903 (2015). https://doi.org/10.1021/jacs.5b07728

70. Y. Yusran, H. Li, X. Guan, D. Li, L. Tang et al., Exfoliated mesoporous 2D covalent organic frameworks for high-rate electrochemical double-layer capacitors. Adv. Mater. 32, 1907289 (2020). https://doi.org/10.1002/adma.201907289

71. D. Deng, K.S. Novoselov, Q. Fu, N. Zheng, Z. Tian et al., Catalysis with two-dimensional materials and their heterostructures. Nat. Nanotechnol. 11, 218-230 (2016). https:// doi.org/10.1038/nnano.2015.340

72. D. Voiry, H. Yamaguchi, J.W. Li, R. Silva, D.C.B. Alves et al., Enhanced catalytic activity in strained chemically exfoliated $\mathrm{WS}_{2}$ nanosheets for hydrogen evolution. Nat. Mater. 12, 850-855 (2013). https://doi.org/10.1038/Nmat3700

73. C. Lee, X.D. Wei, J.W. Kysar, J. Hone, Measurement of the elastic properties and intrinsic strength of monolayer graphene. Science 321, 385-388 (2008). https://doi.org/10.1126/ science. 1157996

74. A. Castellanos-Gomez, M. Poot, G.A. Steele, H.S.J. van der Zant, N. Agrait et al., Elastic properties of freely suspended $\mathrm{MoS}_{2}$ nanosheets. Adv. Mater. 24, 772-775 (2012). https:// doi.org/10.1002/adma.201103965

75. J. Pu, Y. Yomogida, K.K. Liu, L.J. Li, Y. Iwasa et al., Highly flexible $\mathrm{MoS}_{2}$ thin-film transistors with ion gel dielectrics. Nano Lett. 12, 4013-4017 (2012). https://doi.org/10.1021/ $\mathrm{nl} 301335 \mathrm{q}$

76. T.F. Jaramillo, K.P. Jorgensen, J. Bonde, J.H. Nielsen, S. Horch et al., Identification of active edge sites for electrochemical $\mathrm{H}_{2}$ evolution from $\mathrm{MoS}_{2}$ nanocatalysts. Science 317, 100-102 (2007). https://doi.org/10.1126/science.11414 83

77. Y. Zheng, Y. Jiao, Y. Zhu, L.H. Li, Y. Han et al., Hydrogen evolution by a metal-free electrocatalyst. Nat. Commun. 5, 3783 (2014). https://doi.org/10.1038/ncomms4783

78. Y.P. Zhu, C.X. Guo, Y. Zheng, S.Z. Qiao, Surface and interface engineering of noble-metal-free electrocatalysts for efficient energy conversion processes. Acc. Chem. Res. 50, 915-923 (2017). https://doi.org/10.1021/acs.accounts.6b006 35

79. E. Parzinger, B. Miller, B. Blaschke, J.A. Garrido, J.W. Ager et al., Photocatalytic stability of single- and few-layer $\mathrm{MoS}_{2}$. ACS Nano 9, 11302-11309 (2015). https://doi.org/10.1021/ acsnano.5b04979

80. Y. Zheng, Y. Jiao, L. Ge, M. Jaroniec, S.Z. Qiao, Two-step boron and nitrogen doping in graphene for enhanced synergistic catalysis. Angew. Chem. Int. Ed. 52, 3110-3116 (2013). https://doi.org/10.1002/anie.201209548

81. Y. Jiao, Y. Zheng, M. Jaroniec, S.Z. Qiao, Origin of the electrocatalytic oxygen reduction activity of graphene-based 
catalysts: A roadnnap to achieve the best performance. J. Am. Chem. Soc. 136, 4394-4403 (2014). https://doi.org/10.1021/ ja500432h

82. D.H. Deng, X.L. Pan, L.A. Yu, Y. Cui, Y.P. Jiang et al., Toward N-doped graphene via solvothermal synthesis. Chem. Mater. 23, 1188-1193 (2011). https://doi.org/10.1021/cm102 $666 \mathrm{r}$

83. Z.Z. Lin, X.C. Wang, Nanostructure engineering and doping of conjugated carbon nitride semiconductors for hydrogen photosynthesis. Angew. Chem. Int. Ed. 52, 1735-1738 (2013). https://doi.org/10.1002/anie.201209017

84. J.R. Ran, T.Y. Ma, G.P. Gao, X.W. Du, S.Z. Qiao, Porous P-doped graphitic carbon nitride nanosheets for synergistically enhanced visible-light photocatalytic $\mathrm{H}_{2}$ production. Energy Environ. Sci. 8, 3708-3717 (2015). https://doi.org/ 10.1039/c5ee02650d

85. Y.P. Zhu, T.Z. Ren, Z.Y. Yuan, Mesoporous phosphorusdoped $g-\mathrm{C}_{3} \mathrm{~N}_{4}$ nanostructured flowers with superior photocatalytic hydrogen evolution performance. ACS Appl. Mater. Interfaces 7, 16850-16856 (2015). https://doi.org/10.1021/ acsami.5b04947

86. F. Besenbacher, M. Brorson, B.S. Clausen, S. Helveg, B. Hinnemann et al., Recent STM, DFT and HAADF-STEM studies of sulfide-based hydrotreating catalysts: Insight into mechanistic, structural and particle size effects. Catal. Today 130, 86-96 (2008). https://doi.org/10.1016/j.cattod.2007.08. 009

87. J. Bonde, P.G. Moses, T.F. Jaramillo, J.K. Norskov, I. Chorkendorff, Hydrogen evolution on nano-particulate transition metal sulfides. Faraday Discuss 140, 219-231 (2008). https:// doi.org/10.1039/b803857k

88. L.S. Byskov, J.K. Norskov, B.S. Clausen, H. Topsoe, Dft calculations of unpromoted and promoted $\mathrm{MoS}_{2}$-based hydrodesulfurization catalysts. J. Catal. 187, 109-122 (1999). https://doi.org/10.1006/jcat.1999.2598

89. J.V. Lauritsen, J. Kibsgaard, G.H. Olesen, P.G. Moses, B. Hinnemann et al., Location and coordination of promoter atoms in $\mathrm{Co}$ - and $\mathrm{Ni}$-promoted $\mathrm{MoS}_{2}$-based hydrotreating catalysts. J. Catal. 249, 220-233 (2007). https://doi.org/10. 1016/j.jcat.2007.04.013

90. M. Gong, W. Zhou, M.C. Tsai, J.G. Zhou, M.Y. Guan et al., Nanoscale nickel oxide/nickel heterostructures for active hydrogen evolution electrocatalysis. Nat. Commun. 5, 4695 (2014). https://doi.org/10.1038/ncomms5695

91. K.S. Novoselov, A. Mishchenko, A. Carvalho, A.H.C. Neto, 2D materials and van der waals heterostructures. Science 353, aac9439 (2016). https://doi.org/10.1126/science.aac9439

92. W.G. Tu, Y. Zhou, Q. Liu, S.C. Yan, S.S. Bao et al., An in situ simultaneous reduction-hydrolysis technique for fabrication of $\mathrm{TiO}_{2}$-graphene 2D sandwich-like hybrid nanosheets: Graphene-promoted selectivity of photocatalytic-driven hydrogenation and coupling of $\mathrm{CO}_{2}$ into methane and ethane. Adv. Funct. Mater. 23, 1743-1749 (2013). https://doi.org/10.1002/ adfm.201202349

93. J.F. Xie, J.J. Zhang, S. Li, F. Grote, X.D. Zhang et al., Controllable disorder engineering in oxygen-incorporated $\mathrm{MoS}_{2}$ ultrathin nanosheets for efficient hydrogen evolution. J. Am. Chem. Soc. 135, 17881-17888 (2013). https://doi.org/10. 1021/ja408329q

94. D.H. Deng, L. Yu, X.L. Pan, S. Wang, X.Q. Chen et al., Size effect of graphene on electrocatalytic activation of oxygen. Chem. Commun. 47, 10016-10018 (2011). https://doi.org/ 10.1039/c1cc13033a

95. Y. Jia, L.Z. Zhang, A.J. Du, G.P. Gao, J. Chen et al., Defect graphene as a trifunctional catalyst for electrochemical reactions. Adv. Mater. 28, 9532-9538 (2016). https://doi.org/10. 1002/adma.201602912

96. L. Tao, Q. Wang, S. Dou, Z.L. Ma, J. Huo et al., Edge-rich and dopant-free graphene as a highly efficient metal-free electrocatalyst for the oxygen reduction reaction. Chem. Commun. 52, 2764-2767 (2016). https://doi.org/10.1039/c5cc0 $9173 \mathrm{j}$

97. D. Voiry, A. Mohite, M. Chhowalla, Phase engineering of transition metal dichalcogenides. Chem. Soc. Rev. 44, 2702 2712 (2015). https://doi.org/10.1039/c5cs00151j

98. H. Li, C. Tsai, A.L. Koh, L.L. Cai, A.W. Contryman et al., Activating and optimizing $\mathrm{MoS}_{2}$ basal planes for hydrogen evolution through the formation of strained sulphur vacancies. Nat. Mater. 15, 48-53 (2016). https://doi.org/10.1038/ Nmat4465

99. D. Voiry, J. Yang, M. Chhowalla, Recent strategies for improving the catalytic activity of 2D TMD nanosheets toward the hydrogen evolution reaction. Adv. Mater. 28, 6197-6206 (2016). https://doi.org/10.1002/adma.201505597

100. M. Xiao, Z.L. Wang, M.Q. Lyu, B. Luo, S.C. Wang et al., Hollow nanostructures for photocatalysis: Advantages and challenges. Adv. Mater. 31, 1801369 (2019). https://doi.org/ 10.1002/adma.201801369

101. W. Li, N. Jiang, B. Hu, X. Liu, F.Z. Song et al., Electrolyzer design for flexible decoupled water splitting and organic upgrading with electron reservoirs. Chem 4, 637-649 (2018). https://doi.org/10.1016/j.chempr.2017.12.019

102. S.B. Wang, X.C. Wang, Multifunctional metal-organic frameworks for photocatalysis. Small 11, 3097-3112 (2015). https://doi.org/10.1002/smll.201500084

103. F.X. Xiao, J.W. Miao, H.B. Tao, S.F. Hung, H.Y. Wang et al., One-dimensional hybrid nanostructures for heterogeneous photocatalysis and photoelectrocatalysis. Small 11, 21152131 (2015). https://doi.org/10.1002/smll.201402420

104. H.T. Wang, Z.Y. Lu, S.C. Xu, D.S. Kong, J.J. Cha et al., Electrochemical tuning of vertically aligned $\mathrm{MoS}_{2}$ nanofilms and its application in improving hydrogen evolution reaction. Proc. Natl. Acad. Sci. USA 110, 19701-19706 (2013). https://doi.org/10.1073/pnas.1316792110

105. A. Ambrosi, Z. Sofer, M. Pumera, $2 \mathrm{H} \rightarrow 1 \mathrm{~T}$ phase transition and hydrogen evolution activity of $\mathrm{MoS}_{2}, \mathrm{MoSe}_{2}, \mathrm{WS}_{2}$ and $\mathrm{WSe}_{2}$ strongly depends on the $\mathrm{MX}_{2}$ composition. Chem. Commun. 51, 8450-8453 (2015). https://doi.org/10.1039/ c5cc00803d

106. M.Y. Wang, L.J. Cai, Y. Wang, F.C. Zhou, K. Xu et al., Graphene-draped semiconductors for enhanced photocorrosion resistance and photocatalytic properties. J. Am. Chem. Soc. 
139, 4144-4151 (2017). https://doi.org/10.1021/jacs.7b003 41

107. X. Wang, K. Maeda, A. Thomas, K. Takanabe, G. Xin et al., A metal-free polymeric photocatalyst for hydrogen production from water under visible light. Nat. Mater. 8, 76-80 (2009). https://doi.org/10.1038/nmat2317

108. J. Xu, L. Wang, Y.F. Zhu, Decontamination of bisphenol a from aqueous solution by graphene adsorption. Langmuir 28, 8418-8425 (2012). https://doi.org/10.1021/la301476p

109. J. Liu, Y. Liu, N.Y. Liu, Y.Z. Han, X. Zhang et al., Metal-free efficient photocatalyst for stable visible water splitting via a two-electron pathway. Science 347, 970-974 (2015). https:// doi.org/10.1126/science.aaa3145

110. G.G. Zhang, S.H. Zang, X.C. Wang, Layered $\mathrm{Co}(\mathrm{OH})_{2}$ deposited polymeric carbon nitrides for photocatalytic water oxidation. ACS Catal. 5, 941-947 (2015). https://doi.org/10.1021/ cs502002u

111. R. Kuriki, K. Sekizawa, O. Ishitani, K. Maeda, Visible-lightdriven $\mathrm{CO}_{2}$ reduction with carbon nitride: Enhancing the activity of ruthenium catalysts. Angew. Chem. Int. Ed. 54, 2406-2409 (2015). https://doi.org/10.1002/anie.201411170

112. Y. Zheng, L.H. Lin, B. Wang, X.C. Wang, Graphitic carbon nitride polymers toward sustainable photoredox catalysis. Angew. Chem. Int. Ed. 54, 12868-12884 (2015). https://doi. org/10.1002/anie.201501788

113. M. Zhang, W.J. Luo, Z. Wei, W.J. Jiang, D. Liu et al., Separation free $\mathrm{C}_{3} \mathrm{~N}_{4} / \mathrm{SiO}_{2}$ hybrid hydrogels as high active photocatalysts for TOC removal. Appl. Catal. B-Environ. 194, 105-110 (2016). https://doi.org/10.1016/j.apcatb.2016.04. 049

114. M. Zhang, W.Q. Yao, Y.H. Lv, X.J. Bai, Y.F. Liu et al., Enhancement of mineralization ability of $\mathrm{C}_{3} \mathrm{~N}_{4}$ via a lower valence position by a tetracyanoquinodimethane organic semiconductor. J. Mater. Chem. A 2, 11432-11438 (2014). https://doi.org/10.1039/c4ta01471e

115. F. Dong, Z.Y. Wang, Y.J. Sun, W.K. Ho, H.D. Zhang, Engineering the nanoarchitecture and texture of polymeric carbon nitride semiconductor for enhanced visible light photocatalytic activity. J. Colloid Interface Sci. 401, 70-79 (2013). https://doi.org/10.1016/j.jcis.2013.03.034

116. F. Dong, M.Y. Ou, Y.K. Jiang, S. Guo, Z.B. Wu, Efficient and durable visible light photocatalytic performance of porous carbon nitride nanosheets for air purification. Ind. Eng. Chem. Res. 53, 2318-2330 (2014). https://doi.org/10.1021/ ie4038104

117. Y.J. Wang, X.J. Bai, C.S. Pan, J. He, Y.F. Zhu, Enhancement of photocatalytic activity of $\mathrm{Bi}_{2} \mathrm{WO}_{6}$ hybridized with graphite-like $\mathrm{C}_{3} \mathrm{~N}_{4}$. J. Mater. Chem. 22, 11568-11573 (2012). https://doi.org/10.1039/c2jm16873a

118. C.S. Pan, J. Xu, Y.J. Wang, D. Li, Y.F. Zhu, Dramatic activity of $\mathrm{C}_{3} \mathrm{~N}_{4} / \mathrm{BiPO}_{4}$ photocatalyst with core/shell structure formed by self-assembly. Adv. Funct. Mater. 22, 1518-1524 (2012). https://doi.org/10.1002/adfm.201102306

119. D.M. Chen, K.W. Wang, D.G. Xiang, R.L. Zong, W.Q. Yao et al., Significantly enhancement of photocatalytic performances via core-shell structure of $\mathrm{ZnO} @ m p g-\mathrm{C}_{3} \mathrm{~N}_{4}$. Appl.
Catal. B-Environ. 147, 554-561 (2014). https://doi.org/10. 1016/j.apcatb.2013.09.039

120. X.J. Bai, R.L. Zong, C.X. Li, D. Liu, Y.F. Liu et al., Enhancement of visible photocatalytic activity via $\mathrm{Ag} @$ $\mathrm{C}_{3} \mathrm{~N}_{4}$ core-shell plasmonic composite. Appl. Catal. B-Environ. 147, 82-91 (2014). https://doi.org/10.1016/j.apcatb. 2013.08.007

121. Y. Wang, W. Yang, X. Chen, J. Wang, Y. Zhu, Photocatalytic activity enhancement of core-shell structure g- $\mathrm{C}_{3} \mathrm{~N}_{4} @ \mathrm{TiO}_{2}$ via controlled ultrathin $\mathrm{g}-\mathrm{C}_{3} \mathrm{~N}_{4}$ layer. Appl. Catal. B-Environ. 220, 337-347 (2018). https://doi.org/10.1016/j.apcatb.2017. 08.004

122. J.G. Yu, S.H. Wang, J.X. Low, W. Xiao, Enhanced photocatalytic performance of direct $\mathrm{Z}$-scheme $\mathrm{g}-\mathrm{C}_{3} \mathrm{~N}_{4}-\mathrm{TiO}_{2}$ photocatalysts for the decomposition of formaldehyde in air. Phys. Chem. Chem. Phys. 15, 16883-16890 (2013). https://doi.org/ $10.1039 / \mathrm{c} 3 \mathrm{cp} 53131 \mathrm{~g}$

123. Y.P. Yuan, S.W. Cao, Y.S. Liao, L.S. Yin, C. Xue, Red phosphor/g- $\mathrm{C}_{3} \mathrm{~N}_{4}$ heterojunction with enhanced photocatalytic activities for solar fuels production. Appl. Catal. B-Environ. 140, 164-168 (2013). https://doi.org/10.1016/j.apcatb. 2013.04.006

124. W.J. Ong, L.L. Tan, Y.H. Ng, S.T. Yong, S.P. Chai, Graphitic carbon nitride $\left(\mathrm{g}-\mathrm{C}_{3} \mathrm{~N}_{4}\right)$-based photocatalysts for artificial photosynthesis and environmental remediation: Are we a step closer to achieving sustainability? Chem. Rev. 116, 71597329 (2016). https://doi.org/10.1021/acs.chemrev.6b00075

125. H.L. Gao, S.C. Yan, J.J. Wang, Z.G. Zou, Ion coordination significantly enhances the photocatalytic activity of graphiticphase carbon nitride. Dalton T. 43, 8178-8183 (2014). https://doi.org/10.1039/c3dt53224k

126. P. Wu, J. Wang, J. Zhao, L. Guo, F.E. Osterloh, High alkalinity boosts visible light driven $\mathrm{H}_{2}$ evolution activity of $\mathrm{g}-\mathrm{C}_{3} \mathrm{~N}_{4}$ in aqueous methanol. Chem. Commun. 50, 15521-15524 (2014). https://doi.org/10.1039/c4cc08063g

127. S.B. Yang, Y.J. Gong, J.S. Zhang, L. Zhan, L.L. Ma et al., Exfoliated graphitic carbon nitride nanosheets as efficient catalysts for hydrogen evolution under visible light. Adv. Mater. 25, 2452-2456 (2013). https://doi.org/10.1002/adma.20120 4453

128. G.G. Zhang, M.W. Zhang, X.X. Ye, X.Q. Qiu, S. Lin et al., Iodine modified carbon nitride semiconductors as visible light photocatalysts for hydrogen evolution. Adv. Mater. 26, 805-809 (2014). https://doi.org/10.1002/adma.201303611

129. G. Liu, P. Niu, C.H. Sun, S.C. Smith, Z.G. Chen et al., Unique electronic structure induced high photoreactivity of sulfurdoped graphitic $\mathrm{C}_{3} \mathrm{~N}_{4}$. J. Am. Chem. Soc. 132, 11642-11648 (2010). https://doi.org/10.1021/ja103798k

130. Y. Wang, Y. Di, M. Antonietti, H.R. Li, X.F. Chen et al., Excellent visible-light photocatalysis of fluorinated polymeric carbon nitride solids. Chem. Mater. 22, 5119-5121 (2010). https://doi.org/10.1021/cm1019102

131. G.H. Dong, K. Zhao, L.Z. Zhang, Carbon self-doping induced high electronic conductivity and photoreactivity of $\mathrm{g}-\mathrm{C}_{3} \mathrm{~N}_{4}$. Chem. Commun. 48, 6178-6180 (2012). https://doi.org/10. 1039/C2CC32181E 
132. S.C. Yan, Z.S. Li, Z.G. Zou, Photodegradation of rhodamine $\mathrm{b}$ and methyl orange over boron-doped $\mathrm{g}-\mathrm{C}_{3} \mathrm{~N}_{4}$ under visible light irradiation. Langmuir 26, 3894-3901 (2010). https://doi. org/10.1021/la904023j

133. X.F. Chen, J.S. Zhang, X.Z. Fu, M. Antonietti, X.C. Wang, $\mathrm{Fe}-\mathrm{g}-\mathrm{C}_{3} \mathrm{~N}_{4}$-catalyzed oxidation of benzene to phenol using hydrogen peroxide and visible light. J. Am. Chem. Soc. 131, 11658-11659 (2009). https://doi.org/10.1021/ja903923s

134. G.G. Zhang, C.J. Huang, X.C. Wang, Dispersing molecular cobalt in graphitic carbon nitride frameworks for photocatalytic water oxidation. Small 11, 1215-1221 (2015). https:// doi.org/10.1002/smll.201402636

135. Z.X. Ding, X.F. Chen, M. Antonietti, X.C. Wang, Synthesis of transition metal-modified carbon nitride polymers for selective hydrocarbon oxidation. Chemsuschem 4, 274-281 (2011). https://doi.org/10.1002/cssc.201000149

136. T. Xiong, W. Cen, Y. Zhang, F. Dong, Bridging the $g-\mathrm{C}_{3} \mathrm{~N}_{4}$ interlayers for enhanced photocatalysis. ACS Catal. 6, 24622472 (2016). https://doi.org/10.1021/acscatal.5b02922

137. J. Thote, H.B. Aiyappa, A. Deshpande, D. Diaz Diaz, S. Kurungot et al., A covalent organic framework-cadmium sulfide hybrid as a prototype photocatalyst for visible-lightdriven hydrogen production. Chemistry 20, 15961-15965 (2014). https://doi.org/10.1002/chem.201403800

138. B. Zhu, B. Lin, Y. Zhou, P. Sun, Q. Yao et al., Enhanced photocatalytic $\mathrm{H}_{2}$ evolution on $\mathrm{ZnS}$ loaded with graphene and $\mathrm{MoS}_{2}$ nanosheets as cocatalysts. J. Mater. Chem. A 2, 3819-3827 (2014). https://doi.org/10.1039/c3ta14819j

139. U. Maitra, U. Gupta, M. De, R. Datta, A. Govindaraj et al., Highly effective visible-light-induced $\mathrm{H}_{2}$ generation by single-layer $1 \mathrm{~T}-\mathrm{MoS}_{2}$ and a nanocomposite of few-layer $2 \mathrm{H}-\mathrm{MoS}_{2}$ with heavily nitrogenated graphene. Angew. Chem. Int. Ed. 52, 13057-13061 (2013). https://doi.org/10.1002/ anie. 201306918

140. H.N. Kim, T.W. Kim, I.Y. Kim, S.-J. Hwang, Cocatalyst-free photocatalysts for efficient visible-light-induced $\mathrm{H}_{2}$ production: Porous assemblies of CdS quantum dots and layered titanate nanosheets. Adv. Funct. Mater. 21, 3111-3118 (2011). https://doi.org/10.1002/adfm.201100453

141. D. Mullangi, D. Chakraborty, A. Pradeep, V. Koshti, C.P. Vinod et al., Highly stable COF-supported $\mathrm{Co} / \mathrm{Co}(\mathrm{OH})_{2}$ nanoparticles heterogeneous catalyst for reduction of nitrile/ nitro compounds under mild conditions. Small 14, e1801233 (2018). https://doi.org/10.1002/smll.201801233

142. B.J. Yao, J.T. Li, N. Huang, J.L. Kan, L. Qiao et al., Pd NPloaded and covalently cross-linked COF membrane microreactor for aqueous $\mathrm{CBs}$ dechlorination at room temperature. ACS Appl. Mater. Interfaces 10, 20448-20457 (2018). https://doi.org/10.1021/acsami.8b04022

143. T. Banerjee, F. Haase, G. Savasci, K. Gottschling, C. Ochsenfeld et al., Single-site photocatalytic $\mathrm{H}_{2}$ evolution from covalent organic frameworks with molecular cobaloxime co-catalysts. J. Am. Chem. Soc. 139, 16228-16234 (2017). https:// doi.org/10.1021/jacs.7b07489

144. H.B. Aiyappa, J. Thote, D.B. Shinde, R. Banerjee, S. Kurungot, Cobalt-modified covalent organic framework as a robust water oxidation electrocatalyst. Chem. Mater. 28, 4375-4379 (2016). https://doi.org/10.1021/acs.chemmater.6b01370

145. W. Zhong, R. Sa, L. Li, Y. He, L. Li et al., A covalent organic framework bearing single ni sites as a synergistic photocatalyst for selective photoreduction of $\mathrm{CO}_{2}$ to CO. J. Am. Chem. Soc. 141, 7615-7621 (2019). https://doi.org/10.1021/jacs. 9b02997

146. L. Qin, H. Yi, G. Zeng, C. Lai, D. Huang et al., Hierarchical porous carbon material restricted au catalyst for highly catalytic reduction of nitroaromatics. J. Hazard Mater. 380, 120864 (2019). https://doi.org/10.1016/j.jhazmat.2019. 120864

147. I. Roger, M.A. Shipman, M.D. Symes, Earth-abundant catalysts for electrochemical and photoelectrochemical water splitting. Nat. Rev. Chem. 1, 0003 (2017). https://doi.org/10. 1038/s41570-016-0003

148. Z.W. Seh, J. Kibsgaard, C.F. Dickens, I.B. Chorkendorff, J.K. Norskov et al., Combining theory and experiment in electrocatalysis: Insights into materials design. Science 355, aad4998 (2017). https://doi.org/10.1126/science.aad4998

149. N.T. Suen, S.F. Hung, Q. Quan, N. Zhang, Y.J. Xu et al., Electrocatalysis for the oxygen evolution reaction: Recent development and future perspectives. Chem. Soc. Rev. 46, 337-365 (2017). https://doi.org/10.1039/C6CS00328A

150. M. Gong, H.J. Dai, A mini review of NiFe-based materials as highly active oxygen evolution reaction electrocatalysts. Nano Res. 8, 23-39 (2015). https://doi.org/10.1007/ s12274-014-0591-z

151. L.N. Dang, H.F. Liang, J.Q. Zhuo, B.K. Lamb, H.Y. Sheng et al., Direct synthesis and anion exchange of noncarbonate-intercalated $\mathrm{NiFe}$-layered double hydroxides and the influence on electrocatalysis. Chem. Mater. 30, 4321-4330 (2018). https://doi.org/10.1021/acs.chemmater.8b01334

152. C.X. Guo, Y. Zheng, J.R. Ran, F.X. Xie, M. Jaroniec et al., Engineering high-energy interfacial structures for high-performance oxygen-involving electrocatalysis. Angew. Chem. Int. Ed. 56, 8539-8543 (2017). https://doi.org/10.1002/anie. 201701531

153. R. Chen, S.F. Hung, D.J. Zhou, J.J. Gao, C.J. Yang et al., Layered structure causes bulk NiFe layered double hydroxide unstable in alkaline oxygen evolution reaction. Adv. Mater. 31, 1903909 (2019). https://doi.org/10.1002/adma.20190 3909

154. Q. Wang, L. Shang, R. Shi, X. Zhang, Y.F. Zhao et al., NiFe layered double hydroxide nanoparticles on $\mathrm{Co}, \mathrm{N}$-codoped carbon nanoframes as efficient bifunctional catalysts for rechargeable zinc-air batteries. Adv. Energy Mater. 7, 1700467 (2017). https://doi.org/10.1002/aenm.201700467

155. J.T. Zhang, L. Yu, Y. Chen, X.F. Lu, S.Y. Gao et al., Designed formation of double-shelled Ni-Fe layered-double-hydroxide nanocages for efficient oxygen evolution reaction. Adv. Mater. 32, 1906432 (2020). https://doi.org/10.1002/adma. 201906432

156. B. Nagendra, K. Mohan, E.B. Gowd, Polypropylene/layered double hydroxide (LDH) nanocomposites: Influence of LDH particle size on the crystallization behavior of polypropylene. 
ACS Appl. Mater. Interfaces 7, 12399-12410 (2015). https:// doi.org/10.1021/am5075826

157. J. Kosco, I. McCulloch, Residual Pd enables photocatalytic $\mathrm{H}_{2}$ evolution from conjugated polymers. ACS Energy Lett. 3, 2846-2850 (2018). https://doi.org/10.1021/acsenergylett. $8 \mathrm{~b} 01853$

158. R. Chen, C.J. Yang, W.Z. Cai, H.Y. Wang, J.W. Miao et al., Use of platinum as the counter electrode to study the activity of nonprecious metal catalysts for the hydrogen evolution reaction. ACS Energy Lett. 2, 1070-1075 (2017). https://doi. org/10.1021/acsenergylett.7b00219

159. A. Tiwari, T. Maagaard, I. Chorkendorff, S. Horch, Effect of dissolved glassware on the structure-sensitive part of the $\mathrm{Cu}(111)$ voltammogram in $\mathrm{KOH}$. ACS Energy Lett. 4, 16451649 (2019). https://doi.org/10.1021/acsenergylett.9b01064

160. Z.S. Zhang, L. Melo, R.P. Jansonius, F. Habibzadeh, E.R. Grant et al., $\mathrm{Ph}$ matters when reducing $\mathrm{CO}_{2}$ in an electrochemical flow cell. ACS Energy Lett. 5, 3101-3107 (2020). https://doi.org/10.1021/acsenergylett.0c01606

161. H.L. You, Z. Wu, L.H. Zhang, Y.R. Ying, Y. Liu et al., Harvesting the vibration energy of $\mathrm{BiFeO}_{3}$ nanosheets for hydrogen evolution. Angew. Chem. Int. Ed. 58, 11779-11784 (2019). https://doi.org/10.1002/anie.201906181

162. P.V. Kamat, S. Jin, Semiconductor photocatalysis: "Tell us the complete story!" ACS Energy Lett. 3, 622-623 (2018). https://doi.org/10.1021/acsenergylett.8b00196

163. A.S. Hainer, J.S. Hodgins, V. Sandre, M. Vallieres, A.E. Lanterna et al., Photocatalytic hydrogen generation using metal-decorated $\mathrm{TiO}_{2}$ : Sacrificial donors vs true water splitting. ACS Energy Lett. 3, 542-545 (2018). https://doi.org/10. 1021/acsenergylett.8b00152

164. S.F. Hung, Y.P. Zhu, G.Q. Tzeng, H.C. Chen, C.S. Hsu et al., In situ spatially coherent identification of phosphide-based catalysts: Crystallographic latching for highly efficient overall water electrolysis. ACS Energy Lett. 4, 2813-2820 (2019). https://doi.org/10.1021/acsenergylett.9b02075

165. J. Wong, S.T. Omelchenko, H.A. Atwater, Impact of semiconductor band tails and band filling on photovoltaic efficiency limits. ACS Energy Lett. 6, 52-57 (2021). https://doi.org/10. 1021/acsenergylett.0c02362

166. Y. Nosaka, A. Nosaka, Understanding hydroxyl radical $(\bullet \mathrm{OH})$ generation processes in photocatalysis. ACS Energy Lett. 1, 356-359 (2016). https://doi.org/10.1021/acsenergylett.6b001 74

167. D. Salvatore, C.P. Berlinguette, Voltage matters when reducing $\mathrm{CO}_{2}$ in an electrochemical flow cell. ACS Energy Lett. 5, 215-220 (2020). https://doi.org/10.1021/acsenergylett.9b023 56

168. S.Q. Niu, S.W. Li, Y.C. Du, X.J. Han, P. Xu, How to reliably report the overpotential of an electrocatalyst. ACS Energy Lett. 5, 1083-1087 (2020). https://doi.org/10.1021/acsenergyl ett.0c00321

169. S. Anantharaj, S. Kundu, Do the evaluation parameters reflect intrinsic activity of electrocatalysts in electrochemical water splitting? ACS Energy Lett. 4, 1260-1264 (2019). https://doi. org/10.1021/acsenergylett.9b00686
170. V. Nicolosi, M. Chhowalla, M.G. Kanatzidis, M.S. Strano, J.N. Coleman, Liquid exfoliation of layered materials. Science 340, 1226419 (2013). https://doi.org/10.1126/science. 1226419

171. Y. Zheng, Y. Jiao, Y. Zhu, Q. Cai, A. Vasileff et al., Moleculelevel $\mathrm{g}-\mathrm{C}_{3} \mathrm{~N}_{4}$ coordinated transition metals as a new class of electrocatalysts for oxygen electrode reactions. J. Am. Chem. Soc. 139, 3336-3339 (2017). https://doi.org/10.1021/jacs. $6 \mathrm{~b} 13100$

172. T. Su, Q. Shao, Z. Qin, Z. Guo, Z. Wu, Role of interfaces in two-dimensional photocatalyst for water splitting. ACS Catal. 8, 2253-2276 (2018). https://doi.org/10.1021/acscatal.7b034 37

173. J. Sturala, J. Luxa, M. Pumera, Z. Sofer, Chemistry of graphene derivatives: Synthesis, applications, and perspectives. Chem-Eur J. 24, 5992-6006 (2018). https://doi.org/10.1002/ chem. 201704192

174. M.N. Obrovac, V.L. Chevrier, Alloy negative electrodes for Li-ion batteries. Chem. Rev. 114, 11444-11502 (2014). https://doi.org/10.1021/cr500207g

175. P.W. Bridgman, Two new modifications of phosphorus. J. Am. Chem. Soc. 36, 1344-1363 (1914). https://doi.org/10. 1021/ja02184a002

176. C.K. Chan, X.F. Zhang, Y. Cui, High capacity Li ion battery anodes using Ge nanowires. Nano Lett. 8, 307-309 (2008). https://doi.org/10.1021/n10727157

177. H.J. Ying, W.Q. Han, Metallic Sn-based anode materials: Application in high-performance lithium-ion and sodiumion batteries. Adv. Sci. 4, 1700298 (2017). https://doi.org/ 10.1002/advs.201700298

178. X.X. Zuo, J. Zhu, P. Muller-Buschbaum, Y.J. Cheng, Silicon based lithium-ion battery anodes: A chronicle perspective review. Nano Energy 31, 113-143 (2017). https://doi.org/10. 1016/j.nanoen.2016.11.013

179. N. Nitta, G. Yushin, High-capacity anode materials for lithium-ion batteries: Choice of elements and structures for active particles. Part Part Syst. Char. 31, 317-336 (2014). https://doi.org/10.1002/ppsc.201300231

180. G. Abellan, S. Wild, V. Lloret, N. Scheuschner, R. Gillen et al., Fundamental insights into the degradation and stabilization of thin layer black phosphorus. J. Am. Chem. Soc. 139, 10432-10440 (2017). https://doi.org/10.1021/jacs.7b04971

181. O.I. Malyi, K.V. Sopiha, C. Draxl, C. Persson, Stability and electronic properties of phosphorene oxides: From 0-dimensional to amorphous 2-dimensional structures. Nanoscale 9, 2428-2435 (2017). https://doi.org/10.1039/c6nr08810d

182. D. Hanlon, C. Backes, E. Doherty, C.S. Cucinotta, N.C. Berner et al., Liquid exfoliation of solvent-stabilized fewlayer black phosphorus for applications beyond electronics. Nat. Commun. 6, 8563 (2015). https://doi.org/10.1038/ ncomms9563

183. Q. Zhang, S. Huang, J. Deng, D.T. Gangadharan, F. Yang et al., Ice-assisted synthesis of black phosphorus nanosheets as a metal-free photocatalyst: 2D/2D heterostructure for broadband $\mathrm{H}_{2}$ evolution. Adv. Funct. Mater. 29, 1902486 (2019). https://doi.org/10.1002/adfm.201902486 
184. L. Li, Y. Yu, G.J. Ye, Q. Ge, X. Ou et al., Black phosphorus field-effect transistors. Nat. Nanotechnol. 9, 372-377 (2014). https://doi.org/10.1038/nnano.2014.35

185. C. Ashworth, 2D materials: The thick and the thin. Nat. Rev. Mater. 3, 18019 (2018). https://doi.org/10.1038/natrevmats. 2018.19

186. X.Q. Wang, Y.F. Chen, B.J. Zheng, F. Qi, J.R. He et al., Graphene-like $\mathrm{WSe}_{2}$ nanosheets for efficient and stable hydrogen evolution. J. Alloy. Compd. 691, 698-704 (2017). https://doi. org/10.1016/j.jallcom.2016.08.305

187. L. Zhang, X.Q. Ji, X. Ren, Y.J. Ma, X.F. Shi et al., Electrochemical ammonia synthesis via nitrogen reduction reaction on a $\mathrm{MoS}_{2}$ catalyst: Theoretical and experimental studies. Adv. Mater. 30, 1800191 (2018). https://doi.org/10.1002/ adma.201800191

188. A.P. Cote, A.I. Benin, N.W. Ockwig, M. O'Keeffe, A.J. Matzger et al., Porous, crystalline, covalent organic frameworks. Science 310, 1166-1170 (2005). https://doi.org/10. 1126/science. 1120411

189. X. Tan, W. Zeng, Y. Fan, J. Yan, G. Zhao, Covalent organic frameworks bearing pillar[6]arene-reduced Au nanoparticles for the catalytic reduction of nitroaromatics. Nanotechnology 31, 135705 (2020). https://doi.org/10.1088/1361-6528/ab5ff5

190. O. Mashtalir, M. Naguib, V.N. Mochalin, Y. Dall'Agnese, $\mathrm{M}$. Heon et al., Intercalation and delamination of layered carbides and carbonitrides. Nat. Commun. 4, 1716 (2013). https://doi.org/10.1038/ncomms2664

191. D. Geng, X. Zhao, Z. Chen, W. Sun, W. Fu et al., Direct synthesis of large-area $2 \mathrm{D} \mathrm{Mo}_{2} \mathrm{C}$ on in situ grown graphene. Adv. Mater. 29, 1700072 (2017). https://doi.org/10.1002/ adma.201700072

192. M. Naguib, M. Kurtoglu, V. Presser, J. Lu, J.J. Niu et al., Two-dimensional nanocrystals produced by exfoliation of $\mathrm{Ti}_{3} \mathrm{AlC}_{2}$. Adv. Mater. 23, 4248-4253 (2011). https://doi.org/ 10.1002/adma.201102306

193. M. Naguib, O. Mashtalir, J. Carle, V. Presser, J. Lu et al., Two-dimensional transition metal carbides. ACS Nano 6, 1322-1331 (2012). https://doi.org/10.1021/nn204153h

194. M. Ghidiu, M.R. Lukatskaya, M.Q. Zhao, Y. Gogotsi, M.W. Barsoum, Conductive two-dimensional titanium carbide "clay" with high volumetric capacitance. Nature 516, 78-81 (2014). https://doi.org/10.1038/nature13970

195. M. Naguib, Y. Gogotsi, Synthesis of two-dimensional materials by selective extraction. Acc. Chem. Res. 48, 128-135 (2015). https://doi.org/10.1021/ar500346b

196. B. Anasori, Y. Xie, M. Beidaghi, J. Lu, B.C. Hosler et al., Two-dimensional, ordered, double transition metals carbides (MXenes). ACS Nano 9, 9507-9516 (2015). https://doi.org/ 10.1021/acsnano.5b03591

197. J. Halim, M.R. Lukatskaya, K.M. Cook, J. Lu, C.R. Smith et al., Transparent conductive two-dimensional titanium carbide epitaxial thin films. Chem. Mater. 26, 2374-2381 (2014). https://doi.org/10.1021/cm500641a

198. M.W. Barsoum, The $M_{n+1} A_{X}$ phases: A new class of solids; thermodynamically stable nanolaminates. Prog. Solid State
Chem. 28, 201-281 (2000). https://doi.org/10.1016/S00796786(00)00006-6

199. A.N. Enyashin, A.L. Ivanoyskii, Structural and electronic properties and stability of MXenes $\mathrm{Ti}_{2} \mathrm{C}$ and $\mathrm{Ti}_{3} \mathrm{C}_{2}$ functionalized by methoxy groups. J. Phys. Chem. C 117, 1363713643 (2013). https://doi.org/10.1021/jp401820b

200. Q. Tang, Z. Zhou, P.W. Shen, Are MXenes promising anode materials for Li ion batteries? Computational studies on electronic properties and Li storage capability of $\mathrm{Ti}_{3} \mathrm{C}_{2}$ and $\mathrm{Ti}_{3} \mathrm{C}_{2} \mathrm{X}_{2}(\mathrm{X}=\mathrm{F}, \mathrm{OH})$ monolayer. J. Am. Chem. Soc. 134, 16909-16916 (2012). https://doi.org/10.1021/ja308463r

201. Y. Xie, P.R.C. Kent, Hybrid density functional study of structural and electronic properties of functionalized $\mathrm{Ti}_{\mathrm{n}+1} \mathrm{X}_{\mathrm{n}}(\mathrm{X}$ $=$ C, N) monolayers. Phys. Rev. B 87, 235441 (2013). https:// doi.org/10.1103/PhysRevB.87.235441

202. M. Khazaei, M. Arai, T. Sasaki, C.Y. Chung, N.S. Venkataramanan et al., Novel electronic and magnetic properties of two-dimensional transition metal carbides and nitrides. Adv. Funct. Mater. 23, 2185-2192 (2013). https://doi.org/10.1002/ adfm.201202502

203. L.M. Viculis, J.J. Mack, R.B. Kaner, A chemical route to carbon nanoscrolls. Science 299, 1361 (2003). https://doi. org/10.1126/science.1078842

204. M.V. Savoskin, V.N. Mochalin, A.P. Yaroshenko, N.I. Lazareva, T.E. Konstantinova et al., Carbon nanoscrolls produced from acceptor-type graphite intercalation compounds. Carbon 45, 2797-2800 (2007). https://doi.org/10.1016/j.carbon.2007. 09.031

205. A.N. Enyashin, A.L. Ivanovskii, Atomic structure, comparative stability and electronic properties of hydroxylated $\mathrm{Ti}_{2} \mathrm{C}$ and $\mathrm{Ti}_{3} \mathrm{C}_{2}$ nanotubes. Comput. Theor. Chem. 989, 27-32 (2012). https://doi.org/10.1016/j.comptc.2012.02.034

206. M. Kurtoglu, M. Naguib, Y. Gogotsi, M.W. Barsoum, First principles study of two-dimensional early transition metal carbides. MRS Commun. 2, 133-137 (2012). https://doi.org/ 10.1557/mrc.2012.25

207. M. Naguib, J. Come, B. Dyatkin, V. Presser, P.L. Taberna et al., MXene: A promising transition metal carbide anode for lithium-ion batteries. ElectroChem. Commun. 16, 61-64 (2012). https://doi.org/10.1016/j.elecom.2012.01.002

208. J. Come, M. Naguib, P. Rozier, M.W. Barsoum, Y. Gogotsi et al., A non-aqueous asymmetric cell with a $\mathrm{Ti}_{2} \mathrm{C}$-based twodimensional negative electrode. J. Electrochem. Soc. 159, A1368-A1373 (2012). https://doi.org/10.1149/2.003208jes

209. M.R. Lukatskaya, O. Mashtalir, C.E. Ren, Y. Dall'Agnese, P. Rozier et al., Cation intercalation and high volumetric capacitance of two-dimensional titanium carbide. Science 341, 1502-1505 (2013). https://doi.org/10.1126/science. 1241488

210. D.Q. Er, J.W. Li, M. Naguib, Y. Gogotsi, V.B. Shenoy, $\mathrm{Ti}_{3} \mathrm{C}_{2}$ MXene as a high capacity electrode material for metal $(\mathrm{Li}$, $\mathrm{Na}, \mathrm{K}, \mathrm{Ca}$ ) ion batteries. ACS Appl. Mater. Interfaces 6, 11173-11179 (2014). https://doi.org/10.1021/am501144q

211. T.Y. Ma, J.L. Cao, M. Jaroniec, S.Z. Qiao, Interacting carbon nitride and titanium carbide nanosheets for high-performance oxygen evolution. Angew. Chem. Int. Ed. 55, 1138-1142 (2016). https://doi.org/10.1002/anie.201509758 
212. C.Y. Ling, L. Shi, Y.X. Ouyang, Q. Chen, J.L. Wang, Transition metal-promoted $\mathrm{V}_{2} \mathrm{CO}_{2}$ (MXenes): A new and highly active catalyst for hydrogen evolution reaction. Adv. Sci. 3, 1600180 (2016). https://doi.org/10.1002/advs.201600180

213. M.F. Shao, R.K. Zhang, Z.H. Li, M. Wei, D.G. Evans et al., Layered double hydroxides toward electrochemical energy storage and conversion: Design, synthesis and applications. Chem. Commun. 51, 15880-15893 (2015). https://doi.org/ 10.1039/C5CC07296D

214. H.S. Yang, Z.L. Li, B. Lu, J. Gao, X.T. Jin et al., Reconstruction of inherent graphene oxide liquid crystals for large-scale fabrication of structure-intact graphene aerogel bulk toward practical applications. ACS Nano 12, 11407-11416 (2018). https://doi.org/10.1021/acsnano.8b06380

215. R. Liu, Y. Wang, D. Liu, Y. Zou, S. Wang, Water-plasmaenabled exfoliation of ultrathin layered double hydroxide nanosheets with multivacancies for water oxidation. Adv. Mater. 29, 1701546 (2017). https://doi.org/10.1002/adma. 201701546

216. Y. Zhao, X. Zhang, X. Jia, G.I.N. Waterhouse, R. Shi et al., Sub-3 nm ultrafine monolayer layered double hydroxide nanosheets for electrochemical water oxidation. Adv. Energy Mater. 8, 1703585 (2018). https://doi.org/10.1002/aenm. 201703585

217. R. Mohan, Green bismuth. Nat. Chem. 2, 336-336 (2010). https://doi.org/10.1038/nchem.609

218. D.D. Zhu, J.L. Liu, S.Z. Qiao, Recent advances in inorganic heterogeneous electrocatalysts for reduction of carbon dioxide. Adv. Mater. 28, 3423-3452 (2016). https://doi.org/10. 1002/adma.201504766

219. Z.Y. Wang, S. Yan, Y.J. Sun, T. Xiong, F. Dong et al., Bi metal sphere/graphene oxide nanohybrids with enhanced direct plasmonic photocatalysis. Appl. Catal. B-Environ. 214, 148-157 (2017). https://doi.org/10.1016/j.apcatb.2017. 05.040

220. S.M. Beladi-Mousavi, M. Pumera, 2D-pnictogens: Alloybased anode battery materials with ultrahigh cycling stability. Chem. Soc. Rev. 47, 6964-6989 (2018). https://doi.org/ $10.1039 / \mathrm{c} 8 \mathrm{cs} 00425 \mathrm{k}$

221. N. Han, Y. Wang, H. Yang, J. Deng, J.H. Wu et al., Ultrathin bismuth nanosheets from in situ topotactic transformation for selective electrocatalytic $\mathrm{CO}_{2}$ reduction to formate. Nat. Commun. 9, 1320 (2018). https://doi.org/10.1038/ s41467-018-03712-z

222. K. Xu, L. Wang, X. Xu, S.X. Dou, W. Hao et al., Two dimensional bismuth-based layered materials for energy-related applications. Energy Storage Mater. 19, 446-463 (2019). https://doi.org/10.1016/j.ensm.2019.03.021

223. Y.F. Sun, H. Cheng, S. Gao, Q.H. Liu, Z.H. Sun et al., Atomically thick bismuth selenide freestanding single layers achieving enhanced thermoelectric energy harvesting. J. Am. Chem. Soc. 134, 20294-20297 (2012). https://doi.org/10.1021/ja310 2049

224. J. Li, Y. Yu, L. Zhang, Bismuth oxyhalide nanomaterials: Layered structures meet photocatalysis. Nanoscale 6, 84738488 (2014). https://doi.org/10.1039/c4nr02553a
225. J. Di, J. Xia, H. Li, S. Guo, S. Dai, Bismuth oxyhalide layered materials for energy and environmental applications. Nano Energy 41, 172-192 (2017). https://doi.org/10.1016/j. nanoen.2017.09.008

226. J. Li, H. Li, G. Zhan, L. Zhang, Solar water splitting and nitrogen fixation with layered bismuth oxyhalides. Acc. Chem. Res. 50, 112-121 (2017). https://doi.org/10.1021/ acs.accounts.6b00523

227. J.F. Ni, X.X. Bi, Y. Jiang, L. Li, J. Lu, Bismuth chalcogenide compounds $\mathrm{Bi}_{2} \mathrm{X}_{3}(\mathrm{X}=\mathrm{O}, \mathrm{S}, \mathrm{Se})$ : Applications in electrochemical energy storage. Nano Energy 34, 356-366 (2017). https://doi.org/10.1016/j.nanoen.2017.02.041

228. Y.Z. Pei, H. Wang, G.J. Snyder, Band engineering of thermoelectric materials. Adv. Mater. 24, 6125-6135 (2012). https://doi.org/10.1002/adma.201202919

229. R.A. Schlitz, F.G. Brunetti, A.M. Glaudell, P.L. Miller, M.A. Brady et al., Solubility-limited extrinsic n-type doping of a high electron mobility polymer for thermoelectric applications. Adv. Mater. 26, 2825-2830 (2014). https:// doi.org/10.1002/adma.201304866

230. S. Saha, M. Jana, P. Khanra, P. Samanta, H. Koo et al., Band gap engineering of boron nitride by graphene and its application as positive electrode material in asymmetric supercapacitor device. ACS Appl. Mater. Interfaces 7, 14211-14222 (2015). https://doi.org/10.1021/acsami.5b035 62

231. S. Saha, M. Jana, P. Samanta, N.C. Murmu, N.H. Kim et al., Investigation of band structure and electrochemical properties of h-BN/rGO composites for asymmetric supercapacitor applications. Mater. Chem. Phys. 190, 153-165 (2017). https://doi.org/10.1016/j.matchemphys.2017.01.025

232. E.P. Gilshteyn, D. Amanbayev, A.S. Anisimov, T. Kallio, A.G. Nasibulin, All-nanotube stretchable supercapacitor with low equivalent series resistance. Sci. Rep. 7, 17449 (2017). https://doi.org/10.1038/s41598-017-17801-4

233. R. Kumar, S. Sahoo, E. Joanni, R.K. Singh, R.M. Yadav et al., A review on synthesis of graphene, h-BN and $\mathrm{MoS}_{2}$ for energy storage applications: Recent progress and perspectives. Nano Res. 12, 2655-2694 (2019). https://doi.org/10. 1007/s12274-019-2467-8

234. O.M. Yaghi, G.M. Li, H.L. Li, Selective binding and removal of guests in a microporous metal-organic framework. Nature 378, 703-706 (1995). https://doi.org/10.1038/378703a0

235. A.D. Burrows, C.G. Frost, M.F. Mahon, C. Richardson, Postsynthetic modification of tagged metal-organic frameworks. Angew. Chem. Int. Ed. 47, 8482-8486 (2008). https://doi.org/ 10.1002/anie. 200802908

236. Y.F. Song, L. Cronin, Postsynthetic covalent modification of metal-organic framework (MOF) materials. Angew. Chem. Int. Ed. 47, 4635-4637 (2008). https://doi.org/10.1002/anie. 200801631

237. Z.Y. Liu, X.Y. Yang, B.Q. Lu, Z.P. Shi, D.M. Sun et al., Delicate topotactic conversion of coordination polymers to Pd porous nanosheets for high-efficiency electrocatalysis. Appl. Catal. B-Environ. 243, 86-93 (2019). https://doi.org/ 10.1016/j.apcatb.2018.10.028 
238. X.Q. Huang, S.H. Tang, X.L. Mu, Y. Dai, G.X. Chen et al., Freestanding palladium nanosheets with plasmonic and catalytic properties. Nat. Nanotechnol. 6, 28-32 (2011). https:// doi.org/10.1038/Nnano.2010.235

239. H.H. Duan, N. Yan, R. Yu, C.R. Chang, G. Zhou et al., Ultrathin rhodium nanosheets. Nat. Commun. 5, 3093 (2014). https://doi.org/10.1038/ncomms4093

240. P. Kumar, J. Singh, A.C. Pandey, Rational low temperature synthesis and structural investigations of ultrathin bismuth nanosheets. RSC Adv. 3, 2313-2317 (2013). https://doi.org/ $10.1039 / \mathrm{c} 2 \mathrm{ra} 21907 \mathrm{~g}$

241. M. Maillard, P.R. Huang, L. Brus, Silver nanodisk growth by surface plasmon enhanced photoreduction of adsorbed [ $\mathrm{Ag}^{+}$]. Nano Lett. 3, 1611-1615 (2003). https://doi.org/10. 1021/n1034666d

242. I. Washio, Y.J. Xiong, Y.D. Yin, Y.N. Xia, Reduction by the end groups of poly (vinyl pyrrolidone): A new and versatile route to the kinetically controlled synthesis of Ag triangular nanoplates. Adv. Mater. 18, 1745-1749 (2006). https://doi. org/10.1002/adma.200600675

243. Z.X. Fan, Y.H. Zhu, X. Huang, Y. Han, Q.X. Wang et al., Synthesis of ultrathin face-centered-cubic Au@Pt and Au@ $\mathrm{Pd}$ core-shell nanoplates from hexagonal-close-packed $\mathrm{Au}$ square sheets. Angew. Chem. Int. Ed. 54, 5672-5676 (2015). https://doi.org/10.1002/anie.201500993

244. Z.X. Fan, M. Bosman, X. Huang, D. Huang, Y. Yu et al., Stabilization of $4 \mathrm{H}$ hexagonal phase in gold nanoribbons. Nat. Commun. 6, 7684 (2015). https://doi.org/10.1038/ncomm s8684

245. Y. Li, W.X. Wang, K.Y. Xia, W.J. Zhang, Y.Y. Jiang et al., Ultrathin two-dimensional Pd-based nanorings as catalysts for hydrogenation with high activity and stability. Small 11, 4745-4752 (2015). https://doi.org/10.1002/smll.201500769

246. J.L. Zhang, J.M. Du, B.X. Han, Z.M. Liu, T. Jiang et al., Sonochemical formation of single-crystalline gold nanobelts. Angew. Chem. Int. Ed. 45, 1116-1119 (2006). https://doi.org/ 10.1002/anie.200503762

247. Z.X. Fan, X. Huang, C.L. Tan, H. Zhang, Thin metal nanostructures: Synthesis, properties and applications. Chem. Sci. 6, 95-111 (2015). https://doi.org/10.1039/c4sc02571g

248. Y. Chen, Z. Fan, Z. Zhang, W. Niu, C. Li et al., Two-dimensional metal nanomaterials: Synthesis, properties, and applications. Chem. Rev. 118, 6409-6455 (2018). https://doi.org/ 10.1021/acs.chemrev.7b00727

249. M. Chhowalla, D. Voiry, J.E. Yang, H.S. Shin, K.P. Loh, Phase-engineered transition-metal dichalcogenides for energy and electronics. MRS Bull. 40, 585-591 (2015). https://doi. org/10.1557/mrs.2015.142

250. Z.X. Fan, H. Zhang, Crystal phase-controlled synthesis, properties and applications of noble metal nanomaterials. Chem. Soc. Rev. 45, 63-82 (2016). https://doi.org/10.1039/c5cs0 $0467 \mathrm{e}$

251. J. Kim, Y. Lee, S.H. Sun, Structurally ordered FePt nanoparticles and their enhanced catalysis for oxygen reduction reaction. J. Am. Chem. Soc. 132, 4996-4997 (2010). https:// doi.org/10.1021/ja1009629
252. K. Chang, X. Hai, H. Pang, H.B. Zhang, L. Shi et al., Targeted synthesis of $2 \mathrm{H}$ - and 1T-phase $\mathrm{MoS}_{2}$ monolayers for catalytic hydrogen evolution. Adv. Mater. 28, 10033-10041 (2016). https://doi.org/10.1002/adma.201603765

253. Y.D. Qu, H. Medina, S.W. Wang, Y.C. Wang, C.W. Chen et al., Wafer scale phase-engineered $1 \mathrm{~T}$ - and $2 \mathrm{H}-\mathrm{MoSe}_{2} / \mathrm{Mo}$ core-shell 3D-hierarchical nanostructures toward efficient electrocatalytic hydrogen evolution reaction. Adv. Mater. 28, 9831-9838 (2016). https://doi.org/10.1002/adma.201602697

254. M.J. Allen, V.C. Tung, R.B. Kaner, Honeycomb carbon: A review of graphene. Chem. Rev. 110, 132-145 (2010). https:// doi.org/10.1021/cr900070d

255. A. Thomas, A. Fischer, F. Goettmann, M. Antonietti, J.-O. Müller et al., Graphitic carbon nitride materials: Variation of structure and morphology and their use as metal-free catalysts. J. Mater. Chem. 18, 4893-4908 (2008). https://doi.org/ $10.1039 / \mathrm{b} 800274 \mathrm{f}$

256. F. Fina, S.K. Callear, G.M. Carins, J.T.S. Irvine, Structural investigation of graphitic carbon nitride via XRD and neutron diffraction. Chem. Mater. 27, 2612-2618 (2015). https://doi. org/10.1021/acs.chemmater.5b00411

257. B.V. Lotsch, M. Doblinger, J. Sehnert, L. Seyfarth, J. Senker et al., Unmasking melon by a complementary approach employing electron diffraction, solid-state NMR spectroscopy, and theoretical calculations-structural characterization of a carbon nitride polymer. Chem-Eur. J. 13, 4969-4980 (2007). https://doi.org/10.1002/chem.200601759

258. T. Sekine, H. Kanda, Y. Bando, M. Yokoyama, K. Hojou, A graphitic carbon nitride. J. Mater. Sci. Lett. 9, 1376-1378 (1990). https://doi.org/10.1007/Bf00721588

259. Y. Zheng, J. Liu, J. Liang, M. Jaroniec, S.Z. Qiao, Graphitic carbon nitride materials: Controllable synthesis and applications in fuel cells and photocatalysis. Energ. Environ. Sci. 5, 6717-6731 (2012). https://doi.org/10.1039/C2EE03479D

260. Y. Kang, Y. Yang, L.C. Yin, X. Kang, G. Liu et al., An amorphous carbon nitride photocatalyst with greatly extended visible-light-responsive range for photocatalytic hydrogen generation. Adv. Mater. 27, 4572-4577 (2015). https://doi. org/10.1002/adma.201501939

261. L. Cartz, S.R. Srinivasa, R.J. Riedner, J.D. Jorgensen, T.G. Worlton, Effect of pressure on bonding in black phosphorus. J. Chem. Phys. 71, 1718-1721 (1979). https://doi.org/10. $1063 / 1.438523$

262. X. Zhang, H.M. Xie, Z.D. Liu, C.L. Tan, Z.M. Luo et al., Black phosphorus quantum dots. Angew. Chem. Int. Ed. 54, 3653-3657 (2015). https://doi.org/10.1002/anie.201409400

263. H.L. You, Y.M. Jia, Z. Wu, F.F. Wang, H.T. Huang et al., Room-temperature pyro-catalytic hydrogen generation of 2D few-layer black phosphorene under cold-hot alternation. Nat. Commun. 9, 2889 (2018). https://doi.org/10.1038/ s41467-018-05343-w

264. Y. Sun, K. Fujisawa, Z. Lin, Y. Lei, J.S. Mondschein et al., Low-temperature solution synthesis of transition metal dichalcogenide alloys with tunable optical properties. J. Am. Chem. Soc. 139, 11096-11105 (2017). https://doi.org/10. 1021/jacs.7b04443 
265. X. Feng, X.S. Ding, D.L. Jiang, Covalent organic frameworks. Chem. Soc. Rev. 41, 6010-6022 (2012). https://doi. org/10.1039/C2CS35157A

266. S.Y. Ding, W. Wang, Covalent organic frameworks (COFs): From design to applications. Chem. Soc. Rev. 42, 548-568 (2013). https://doi.org/10.1039/C2CS35072F

267. M. Naguib, V.N. Mochalin, M.W. Barsoum, Y. Gogotsi, 25th anniversary article: MXenes: A new family of twodimensional materials. Adv. Mater. 26, 992-1005 (2014). https://doi.org/10.1002/adma.201304138

268. V. Rives, M.A. Ulibarri, Layered double hydroxides (LDH) intercalated with metal coordination compounds and oxometalates. Coord. Chem. Rev. 181, 61-120 (1999). https:// doi.org/10.1016/S0010-8545(98)00216-1

269. A.I. Khan, D. O'Hare, Intercalation chemistry of layered double hydroxides: Recent developments and applications. J. Mater. Chem. 12, 3191-3198 (2002). https://doi.org/10. 1039/b204076j

270. R.Z. Ma, Z.P. Liu, L. Li, N. Iyi, T. Sasaki, Exfoliating layered double hydroxides in formamide: A method to obtain positively charged nanosheets. J. Mater. Chem. 16, 38093813 (2006). https://doi.org/10.1039/b605422f

271. Y. Wang, Y. Zhang, Z. Liu, C. Xie, S. Feng et al., Layered double hydroxide nanosheets with multiple vacancies obtained by dry exfoliation as highly efficient oxygen evolution electrocatalysts. Angew. Chem. Int. Ed. 56, 58675871 (2017). https://doi.org/10.1002/anie.201701477

272. H.F. Feng, Y. Du, C. Wang, W.C. Hao, Efficient visiblelight photocatalysts by constructing dispersive energy band with anisotropic $\mathrm{p}$ and $\mathrm{s}-\mathrm{p}$ hybridization states. Curr. Opin. Green Sust. 6, 93-100 (2017). https://doi.org/10.1016/j. cogsc.2017.05.008

273. Z.F. Xu, K. Xu, H.F. Feng, Y. Du, W.C. Hao, S-p orbital hybridization: A strategy for developing efficient photocatalysts with high carrier mobility. Sci. Bull. 63, 465-468 (2018). https://doi.org/10.1016/j.scib.2018.02.020

274. D.D. Cui, L. Wang, Y. Du, W.C. Hao, J. Chen, Photocatalytic reduction on bismuth-based p-block semiconductors. ACS Sustain. Chem. Eng. 6, 15936-15953 (2018). https:// doi.org/10.1021/acssuschemeng.8b04977

275. J. Lu, W. Zhou, X. Zhang, G. Xiang, Electronic structures and lattice dynamics of layered $\mathrm{BiOCl}$ single crystals. J. Phys. Chem. Lett. 11, 1038-1044 (2020). https://doi.org/ 10.1021/acs.jpclett.9b03575

276. Q.H. Weng, B.J. Wang, X.B. Wang, N. Hanagata, X. Li et al., Highly water-soluble, porous, and biocompatible boron nitrides for anticancer drug delivery. ACS Nano 8, 6123-6130 (2014). https://doi.org/10.1021/nn5014808

277. M. Cai, Q. Liu, Z. Xue, Y. Li, Y. Fan et al., Constructing 2D MOFs from 2D LDHs: A highly efficient and durable electrocatalyst for water oxidation. J. Mater. Chem. A 8, 190-195 (2020). https://doi.org/10.1039/c9ta09397d

278. G. Givaja, P. Amo-Ochoa, C.J. Gomez-Garcia, F. Zamora, Electrical conductive coordination polymers. Chem. Soc. Rev. 41, 115-147 (2012). https://doi.org/10.1039/c1cs1 $5092 \mathrm{~h}$
279. C.H. Hendon, D. Tiana, A. Walsh, Conductive metal-organic frameworks and networks: Fact or fantasy? Phys. Chem. Chem. Phys. 14, 13120-13132 (2012). https://doi.org/10. 1039/c2cp41099k

280. A. Dhakshinamoorthy, A.M. Asiri, H. Garcia, 2D metalorganic frameworks as multifunctional materials in heterogeneous catalysis and electro/photocatalysis. Adv. Mater. 31, 1900617 (2019). https://doi.org/10.1002/adma.201900617

281. Z. Wang, J. Huang, J. Mao, Q. Guo, Z. Chen et al., Metalorganic frameworks and their derivatives with graphene composites: Preparation and applications in electrocatalysis and photocatalysis. J. Mater. Chem. A 8, 2934-2961 (2020). https://doi.org/10.1039/c9ta12776c

282. M. Yi, Z.G. Shen, A review on mechanical exfoliation for the scalable production of graphene. J. Mater. Chem. A 3, 11700-11715 (2015). https://doi.org/10.1039/c5ta00252d

283. X. Ren, J. Zhou, X. Qi, Y. Liu, Z. Huang et al., Few-layer black phosphorus nanosheets as electrocatalysts for highly efficient oxygen evolution reaction. Adv. Energy Mater. 7, 1700396 (2017). https://doi.org/10.1002/aenm.201700396

284. C.X. Zheng, L. Yu, L. Zhu, J.L. Collins, D. Kim et al., Room temperature in-plane ferroelectricity in van der waals $\mathrm{In}_{2} \mathrm{Se}_{3}$. Sci. Adv. 4, eaar7720 (2018). https://doi.org/10.1126/sciadv. aar7720

285. B. Jayasena, S. Subbiah, A novel mechanical cleavage method for synthesizing few-layer graphenes. Nanoscale Res. Lett. 6, 95 (2011). https://doi.org/10.1186/1556-276x-6-95

286. A. Kondo, C.C. Tiew, F. Moriguchi, K. Maeda, Fabrication of metal-organic framework nanosheets and nanorolls with $\mathrm{N}$-donor type bridging ligands. Dalton Trans. 42, 1526715270 (2013). https://doi.org/10.1039/c3dt52130c

287. Z.W. Seh, K.D. Fredrickson, B. Anasori, J. Kibsgaard, A.L. Strickler et al., Two-dimensional molybdenum carbide (MXene) as an efficient electrocatalyst for hydrogen evolution. ACS Energy Lett. 1, 589-594 (2016). https://doi.org/10. 1021/acsenergylett.6b00247

288. Y. Peng, Y.S. Li, Y.J. Ban, H. Jin, W.M. Jiao et al., Metalorganic framework nanosheets as building blocks for molecular sieving membranes. Science 346, 1356-1359 (2014). https://doi.org/10.1126/science.1254227

289. K. Rui, G. Zhao, Y. Chen, Y. Lin, Q. Zhou et al., Hybrid 2D dual-metal-organic frameworks for enhanced water oxidation catalysis. Adv. Funct. Mater. 28, 1801554 (2018). https://doi. org/10.1002/adfm.201801554

290. J. Di, J. Xia, H. Li, Z. Liu, Freestanding atomically-thin twodimensional materials beyond graphene meeting photocatalysis: Opportunities and challenges. Nano Energy 35, 79-91 (2017). https://doi.org/10.1016/j.nanoen.2017.03.030

291. K.S. Novoselov, A.K. Geim, S.V. Morozov, D. Jiang, Y. Zhang et al., Electric field effect in atomically thin carbon films. Science 306, 666-669 (2004). https://doi.org/10.1126/ science. 1102896

292. T.A. Shifa, F. Wang, Y. Liu, J. He, Heterostructures based on 2D materials: A versatile platform for efficient catalysis. Adv. Mater. 31, 1804828 (2019). https://doi.org/10.1002/ adma. 201804828 
293. X. Cai, Y. Luo, B. Liu, H.M. Cheng, Preparation of 2D material dispersions and their applications. Chem. Soc. Rev. 47, 6224-6266 (2018). https://doi.org/10.1039/c8cs00254a

294. J.M. Englert, C. Dotzer, G. Yang, M. Schmid, C. Papp et al., Covalent bulk functionalization of graphene. Nat. Chem. 3, 279-286 (2011). https://doi.org/10.1038/nchem.1010

295. D. Voiry, M. Salehi, R. Silva, T. Fujita, M. Chen et al., Conducting $\mathrm{MoS}_{2}$ nanosheets as catalysts for hydrogen evolution reaction. Nano Lett. 13, 6222-6227 (2013). https://doi.org/ $10.1021 / \mathrm{n} 1403661 \mathrm{~s}$

296. P.L. Cullen, K.M. Cox, M.K. Bin Subhan, L. Picco, O.D. Payton et al., Ionic solutions of two-dimensional materials. Nat. Chem. 9, 244-249 (2017). https://doi.org/10.1038/nchem. 2650

297. N. Zhang, M.Q. Yang, S.Q. Liu, Y.G. Sun, Y.J. Xu, Waltzing with the versatile platform of graphene to synthesize composite photocatalysts. Chem. Rev. 115, 10307-10377 (2015). https://doi.org/10.1021/acs.chemrev.5b00267

298. Z. Wang, G. Wang, H. Qi, M. Wang, M. Wang et al., Ultrathin two-dimensional conjugated metal-organic framework single-crystalline nanosheets enabled by surfactant-assisted synthesis. Chem. Sci. 11, 7665-7671 (2020). https://doi.org/ 10.1039/d0sc01408g

299. M.L. Sushko, J. Liu, Surfactant two-dimensional self-assembly under confinement. J. Phys. Chem. B 115, 4322-4328 (2011). https://doi.org/10.1021/jp2003497

300. L. Song, L. Ci, H. Lu, P.B. Sorokin, C. Jin et al., Large scale growth and characterization of atomic hexagonal boron nitride layers. Nano Lett. 10, 3209-3215 (2010). https://doi. org/10.1021/nl1022139

301. D. Geng, G. Yu, Liquid catalysts: An innovative solution to 2D materials in CVD processes. Mater. Horiz. 5, 1021-1034 (2018). https://doi.org/10.1039/c8mh01088a

302. Y. Shi, H. Li, L.J. Li, Recent advances in controlled synthesis of two-dimensional transition metal dichalcogenides via vapour deposition techniques. Chem. Soc. Rev. 44, 27442756 (2015). https://doi.org/10.1039/c4cs00256c

303. G.W. Shim, W. Hong, S.Y. Yang, S.-Y. Choi, Tuning the catalytic functionality of transition metal dichalcogenides grown by chemical vapour deposition. J. Mater. Chem. A 5, 14950-14968 (2017). https://doi.org/10.1039/c7ta03039h

304. X.J. Wu, X. Huang, J. Liu, H. Li, J. Yang et al., Two-dimensional $\mathrm{CuSe}$ nanosheets with microscale lateral size: Synthesis and template-assisted phase transformation. Angew. Chem. Int. Ed. 53, 5083-5087 (2014). https://doi.org/10. 1002/anie.201311309

305. M. Adnan, J.J. Baumberg, G. Vijaya Prakash, Linear and nonlinear optical probing of various excitons in 2D inorganicorganic hybrid structures. Sci. Rep. 10, 2615 (2020). https:// doi.org/10.1038/s41598-020-59457-7

306. Z. Zhu, Y. Yang, Y. Guan, J. Xue, L. Cui, Construction of a cobalt-embedded nitrogen-doped carbon material with the desired porosity derived from the confined growth of MOFs within graphene aerogels as a superior catalyst towards HER and ORR. J. Mater. Chem. A 4, 15536-15545 (2016). https:// doi.org/10.1039/c6ta05196k
307. F.L. Li, P. Wang, X. Huang, D.J. Young, H.F. Wang et al., Large-scale, bottom-up synthesis of binary metal-organic framework nanosheets for efficient water oxidation. Angew. Chem. Int. Ed. 58, 7051-7056 (2019). https://doi.org/10. 1002/anie.201902588

308. W. Han, K. Liu, S. Yang, F. Wang, J. Su et al., Salt-assisted chemical vapor deposition of two-dimensional materials. Sci. China Chem. 62, 1300-1311 (2019). https://doi.org/ 10.1007/s11426-019-9525-y

309. J. Su, G.D. Li, X.H. Li, J.S. Chen, 2D/2D heterojunctions for catalysis. Adv. Sci. 6, 1801702 (2019). https://doi.org/ 10.1002/advs.201801702

310. H. Li, J.M.T. Wu, X. Huang, G. Lu, J. Yang et al., Rapid and reliable thickness identification of two-dimensional nanosheets using optical microscopy. ACS Nano 7, 1034410353 (2013). https://doi.org/10.1021/nn4047474

311. J.D. Wood, S.A. Wells, D. Jariwala, K.S. Chen, E. Cho et al., Effective passivation of exfoliated black phosphorus transistors against ambient degradation. Nano Lett. 14, 6964-6970 (2014). https://doi.org/10.1021/nl5032293

312. Y. Du, J.C. Zhuang, H.S. Liu, X. Xu, S. Eilers et al., Tuning the band gap in silicene by oxidation. ACS Nano 8 , 10019-10025 (2014). https://doi.org/10.1021/nn504451t

313. Y.C. Lin, R.K. Ghosh, R. Addou, N. Lu, S.M. Eichfeld et al., Atomically thin resonant tunnel diodes built from synthetic van der waals heterostructures. Nat. Commun. 6, 7311 (2015). https://doi.org/10.1038/ncomms8311

314. H. Li, G. Lu, Z.Y. Yin, Q.Y. He, H. Li et al., Optical identification of single- and few-layer $\mathrm{MoS}_{2}$ sheets. Small 8 , 682-686 (2012). https://doi.org/10.1002/smll.201101958

315. M.H. Chiu, M.Y. Li, W.J. Zhang, W.T. Hsu, W.H. Chang et al., Spectroscopic signatures for interlayer coupling in $\mathrm{MoS}_{2}-\mathrm{WSe}_{2}$ van der waals stacking. ACS Nano 8, 96499656 (2014). https://doi.org/10.1021/nn504229z

316. C.L. Tan, P. Yu, Y.L. Hu, J.Z. Chen, Y. Huang et al., Highyield exfoliation of ultrathin two-dimensional ternary chalcogenide nanosheets for highly sensitive and selective fluorescence DNA sensors. J. Am. Chem. Soc. 137, 10430-10436 (2015). https://doi.org/10.1021/jacs.5b06982

317. Z.L. Wang, Transmission electron microscopy of shapecontrolled nanocrystals and their assemblies. J. Phys. Chem. B 104, 1153-1175 (2000). https://doi.org/10.1021/ jp993593c

318. H. Jung, K.M. Cho, K.H. Kim, H.W. Yoo, A. Al-Saggaf et al., Highly efficient and stable $\mathrm{CO}_{2}$ reduction photocatalyst with a hierarchical structure of mesoporous $\mathrm{TiO}_{2}$ on $3 \mathrm{D}$ graphene with few-layered $\mathrm{MoS}_{2}$. ACS Sustain. Chem. Eng. 6, 57185724 (2018). https://doi.org/10.1021/acssuschemeng.8b000 02

319. L.A. Bottomley, J.E. Coury, P.N. First, Scanning probe microscopy. Anal. Chem. 68, R185-R230 (1996). https:// doi.org/10.1021/a1960008+

320. X.L. Chen, Y.Y. Wu, Z.F. Wu, Y. Han, S.G. Xu et al., Highquality sandwiched black phosphorus heterostructure and its quantum oscillations. Nat. Commun. (2015). https://doi.org/ $10.1038 /$ ncomms 8315 
321. C.X. Zheng, Z.Q. Xu, Q.H. Zhang, M.T. Edmonds, K. Watanabe et al., Profound effect of substrate hydroxylation and hydration on electronic and optical properties of monolayer $\mathrm{MoS}_{2}$. Nano Lett. 15, 3096-3102 (2015). https:// doi.org/10.1021/acs.nanolett.5b00098

322. A. Castellanos-Gomez, E. Cappelluti, R. Roldan, N. Agrait, F. Guinea et al., Electric-field screening in atomically thin layers of $\mathrm{MoS}_{2}$ : The role of interlayer coupling. Adv. Mater. 25, 899-903 (2013). https://doi.org/10.1002/adma.20120 3731

323. K. Xu, P.G. Cao, J.R. Heath, Graphene visualizes the first water adlayers on mica at ambient conditions. Science 329, 1188-1191 (2010). https://doi.org/10.1126/science.1192907

324. C. Lee, H. Yan, L.E. Brus, T.F. Heinz, J. Hone et al., Anomalous lattice vibrations of single- and few-layer $\mathrm{MoS}_{2}$. ACS Nano 4, 2695-2700 (2010). https://doi.org/10.1021/nn100 3937

325. Y.F. Sun, Q.H. Liu, S. Gao, H. Cheng, F.C. Lei et al., Pits confined in ultrathin cerium(IV) oxide for studying catalytic centers in carbon monoxide oxidation. Nat. Commun. 4, 2899 (2013). https://doi.org/10.1038/ncomms3899

326. X. Zhang, X.F. Qiao, W. Shi, J.B. Wu, D.S. Jiang et al., Phonon and Raman scattering of two-dimensional transition metal dichalcogenides from monolayer, multilayer to bulk material. Chem. Soc. Rev. 44, 2757-2785 (2015). https://doi. org/10.1039/C4CS00282B

327. G. Eda, H. Yamaguchi, D. Voiry, T. Fujita, M.W. Chen et al., Photoluminescence from chemically exfoliated $\mathrm{MoS}_{2}$. Nano Lett. 11, 5111-5116 (2011). https://doi.org/10.1021/nl201 $874 w$

328. Y.F. Sun, Z.H. Sun, S. Gao, H. Cheng, Q.H. Liu et al., Fabrication of flexible and freestanding zinc chalcogenide single layers. Nat. Commun. 3, 1057 (2012). https://doi.org/10. 1038/ncomms2066

329. W.Q. Qian, Z. Wu, Y.M. Jia, Y.T. Hong, X.L. Xu et al., Thermo-electrochemical coupling for room temperature thermocatalysis in pyroelectric $\mathrm{ZnO}$ nanorods. ElectroChem. Commun. 81, 124-127 (2017). https://doi.org/10.1016/j.elecom.2017.06.017

330. Y.T. Xia, Y.M. Jia, W.Q. Qian, X.L. Xu, Z. Wu et al., Pyroelectrically induced pyro-electro-chemical catalytic activity of $\mathrm{BaTiO}_{3}$ nanofibers under room-temperature cold-hot cycle excitations. Metals-Basel. 7, 122 (2017). https://doi.org/10. 3390/met7040122

331. M. Gavrilescu, K. Demnerova, J. Aamand, S. Agathos, F. Fava, Emerging pollutants in the environment: Present and future challenges in biomonitoring, ecological risks and bioremediation. New Biotechnol. 32, 147-156 (2015). https:// doi.org/10.1016/j.nbt.2014.01.001

332. J. Jiang, K. Zhao, X. Xiao, L. Zhang, Synthesis and facetdependent photoreactivity of biocl single-crystalline nanosheets. J. Am. Chem. Soc. 134, 4473-4476 (2012). https://doi.org/10.1021/ja210484t

333. S. Wu, J. Xiong, J. Sun, Z.D. Hood, W. Zeng et al., Hydroxyldependent evolution of oxygen vacancies enables the regeneration of biocl photocatalyst. ACS Appl. Mater. Interfaces 9,
16620-16626 (2017). https://doi.org/10.1021/acsami.7b017 01

334. Y. Zheng, Z. Yu, H. Ou, A.M. Asiri, Y. Chen et al., Black phosphorus and polymeric carbon nitride heterostructure for photoinduced molecular oxygen activation. Adv. Funct. Mater. 28, 1705407 (2018). https://doi.org/10.1002/adfm. 201705407

335. S.-H. Liu, W.-T. Tang, P.-H. Chou, Microwave-assisted synthesis of triple $2 \mathrm{D} \mathrm{g}-\mathrm{C}_{3} \mathrm{~N}_{4} / \mathrm{Bi}_{2} \mathrm{WO}_{6} / \mathrm{rGO}$ composites for ibuprofen photodegradation: Kinetics, mechanism and toxicity evaluation of degradation products. Chem. Eng. J. 387, 124098 (2020). https://doi.org/10.1016/j.cej.2020.124098

336. Z.N. Norvill, A. Toledo-Cervantes, S. Blanco, A. Shilton, B. Guieysse et al., Photodegradation and sorption govern tetracycline removal during wastewater treatment in algal ponds. Bioresour. Technol. 232, 35-43 (2017). https://doi.org/10. 1016/j.biortech.2017.02.011

337. D. Zhang, S. Xu, X. Zhao, W. Qian, C.R. Bowen et al., Wireless monitoring of small strains in intelligent robots via a joule heating effect in stretchable graphene-polymer nanocomposites. Adv. Funct. Mater. 30, 1910809 (2020). https:// doi.org/10.1002/adfm.201910809

338. X. Zhao, D. Zhang, S. Xu, W. Qian, W. Han et al., Stretching-enhanced triboelectric nanogenerator for efficient wind energy scavenging and ultrasensitive strain sensing. Nano Energy 75, 104920 (2020). https://doi.org/10.1016/j.nanoen. 2020.104920

339. X. Zhao, D. Zhang, S. Xu, W. Qian, W. Han et al., Self-powered wireless monitoring of obstacle position and state in gas pipe via flow-driven triboelectric nanogenerators. Adv. Mater. Technol. 5, 2000466 (2020). https://doi.org/10.1002/admt.202000466

340. C. Zhang, Y.M. Shi, Y.F. Yu, Y.H. Du, B. Zhang, Engineering sulfur defects, atomic thickness, and porous structures into cobalt sulfide nanosheets for efficient electrocatalytic alkaline hydrogen evolution. ACS Catal. 8, 8077-8083 (2018). https:// doi.org/10.1021/acscatal.8b02056

341. S. Hu, M. Zhu, Ultrathin two-dimensional semiconductors for photocatalysis in energy and environment applications. ChemCatChem 11, 6147-6165 (2019). https://doi.org/10. 1002/cctc. 201901597

342. F. Song, X.L. Hu, Ultrathin cobalt-manganese layered double hydroxide is an efficient oxygen evolution catalyst. J. Am. Chem. Soc. 136, 16481-16484 (2014). https://doi.org/10. 1021/ja5096733

343. H.J. Yin, Z.Y. Tang, Ultrathin two-dimensional layered metal hydroxides: An emerging platform for advanced catalysis, energy conversion and storage. Chem. Soc. Rev. 45, 48734891 (2016). https://doi.org/10.1039/c6cs00343e

344. X.K. Kong, C.L. Zhang, S.Y. Hwang, Q.W. Chen, Z.M. Peng, Free-standing holey $\mathrm{Ni}(\mathrm{OH})_{2}$ nanosheets with enhanced activity for water oxidation. Small 13, 1700334 (2017). https://doi.org/10.1002/smll.201700334

345. F. Song, X. Hu, Exfoliation of layered double hydroxides for enhanced oxygen evolution catalysis. Nat. Commun. 5, 4477 (2014). https://doi.org/10.1038/ncomms5477 
346. M.L. Qin, S.M. Li, Y.Z. Zhao, C.Y. Lao, Z.L. Zhang et al., Unprecedented synthesis of holey 2D layered double hydroxide nanomesh for enhanced oxygen evolution. Adv. Energy Mater. 9, 1803060 (2019). https://doi.org/10.1002/aenm.201803060

347. Q. Niu, J. Guo, Y. Tang, X. Guo, J. Nie et al., Sandwich-type bimetal-organic frameworks/graphene oxide derived porous nanosheets doped $\mathrm{Fe} / \mathrm{Co}-\mathrm{N}$ active sites for oxygen reduction reaction. Electrochim. Acta 255, 72-82 (2017). https://doi. org/10.1016/j.electacta.2017.09.125

348. S. Xu, S. Chansai, Y. Shao, S. Xu, Y.-C. Wang et al., Mechanistic study of non-thermal plasma assisted $\mathrm{CO}_{2}$ hydrogenation over $\mathrm{Ru}$ supported on $\mathrm{MgAl}$ layered double hydroxide. Appl. Catal. B-Environ. 268, 118752 (2020). https://doi.org/ 10.1016/j.apcatb.2020.118752

349. M. Asadi, K. Kim, C. Liu, A.V. Addepalli, P. Abbasi et al., Nanostructured transition metal dichalcogenide electrocatalysts for $\mathrm{CO}_{2}$ reduction in ionic liquid. Science 353, 467-470 (2016). https://doi.org/10.1126/science.aaf4767

350. L. Ye, Y. Gao, S. Cao, H. Chen, Y. Yao et al., Assembly of highly efficient photocatalytic $\mathrm{CO}_{2}$ conversion systems with ultrathin two-dimensional metal-organic framework nanosheets. Appl. Catal. B-Environ. 227, 54-60 (2018). https://doi.org/10.1016/j.apcatb.2018.01.028

351. Y. Zhao, N. Liu, S. Zhou, J. Zhao, Two-dimensional ZnO for the selective photoreduction of $\mathrm{CO}_{2}$. J. Mater. Chem. A 7, 16294-16303 (2019). https://doi.org/10.1039/c9ta04477a

352. Y. Hao, Z.L. Dong, M.C. Chen, Y. Chao, Z. Liu et al., Near-infrared light and glucose dual-responsive cascading hydroxyl radical generation for in situ gelation and effective breast cancer treatment. Biomaterials 228, 119568 (2020). https://doi.org/10.1016/j.biomaterials.2019.119568

353. Z.L. Dong, Z.J. Yang, Y. Hao, L.Z. Feng, Fabrication of $\mathrm{H}_{2} \mathrm{O}_{2}$-driven nanoreactors for innovative cancer treatments. Nanoscale 11, 16164-16186 (2019). https://doi.org/10.1039/ c9nr04418c

354. G.Y. Chen, H.L. Qju, P.N. Prasad, X.Y. Chen, Upconversion nanoparticles: Design, nanochemistry, and applications in theranostics. Chem. Rev. 114, 5161-5214 (2014). https:// doi.org/10.1021/cr400425h

355. J.A. Barreto, W. O’Malley, M. Kubeil, B. Graham, H. Stephan et al., Nanomaterials: Applications in cancer imaging and therapy. Adv. Mater. 23, H18-H40 (2011). https://doi. org/10.1002/adma.201100140

356. Z.J. Zhou, J.B. Song, L.M. Nie, X.Y. Chen, Reactive oxygen species generating systems meeting challenges of photodynamic cancer therapy. Chem. Soc. Rev. 45, 6597-6626 (2016). https://doi.org/10.1039/c6cs00271d

357. X.Z. Ai, C.J.H. Ho, J. Aw, A.B.E. Attia, J. Mu et al., In vivo covalent cross-linking of photon-converted rare-earth nanostructures for tumour localization and theranostics. Nat. Commun. 7, 10432 (2016). https://doi.org/10.1038/ncomms 10432

358. Q. Chen, L.G. Xu, C. Liang, C. Wang, R. Peng et al., Photothermal therapy with immune-adjuvant nanoparticles together with checkpoint blockade for effective cancer immunotherapy. Nat. Commun. 7, 13193 (2016). https://doi.org/10. 1038/ncomms 13193

359. M. Ferrari, Cancer nanotechnology: Opportunities and challenges. Nat. Rev. Cancer 5, 161-171 (2005). https://doi.org/ $10.1038 / \mathrm{nrc} 1566$

360. Q.Q. Miao, K.Y. Pu, Organic semiconducting agents for deeptissue molecular imaging: Second near-infrared fluorescence, self-luminescence, and photoacoustics. Adv. Mater. 30, 1801778 (2018). https://doi.org/10.1002/adma.201801778

361. Z.Y. Shen, J.B. Song, B.C. Yung, Z.J. Zhou, A.G. Wu et al., Emerging strategies of cancer therapy based on ferroptosis. Adv. Mater. 30, 1704007 (2018). https://doi.org/10.1002/ adma.201704007

362. M.M. Wu, Y.M. Xue, N. Li, H.Y. Zhao, B. Lei et al., Tumormicroenvironment-induced degradation of ultrathin gadolinium oxide nanoscrolls for magnetic-resonance-imagingmonitored, activatable cancer chemotherapy. Angew. Chem. Int. Ed. 58, 6880-6885 (2019). https://doi.org/10.1002/anie. 201812972

363. J.L. Xia, Y.M.L. Xue, L.L. Xu, M.Z. Sun, N. Li et al., Multimodal channel cancer chemotherapy by $2 \mathrm{D}$ functional gadolinium metal-organic framework. Natl. Sci. Rev. (2020). https://doi.org/10.1093/nsr/nwaa221/5900995

364. C. Dai, R.Z. Hu, C.M. Wang, Z. Liu, S.J. Zhang et al., Defect engineering of $2 \mathrm{D} \mathrm{BiOCl}$ nanosheets for photonic tumor ablation. Nanoscale Horiz. 5, 857-868 (2020). https://doi.org/10. 1039/c9nh00707e 